JOURNAL OF

SYMPLECTIC GEOMETRY

Volume 3, Number 4, 589-654, 2005

\title{
COMPACTNESS FOR PUNCTURED HOLOMORPHIC CURVES
}

\author{
K. Cieliebak and K. Mohnke
}

\begin{abstract}
Bourgeois, Eliashberg, Hofer, Wysocki and Zehnder recently proved a general compactness result for moduli spaces of punctured holomorphic curves arising in symplectic field theory. In this paper we present an alternative proof of this result. The main idea is to determine a priori the levels at which holomorphic curves split, thus reducing the proof to two separate cases: long cylinders of small area, and regions with compact image. The second case requires a generalization of Gromov compactness for holomorphic curves with free boundary.
\end{abstract}

\section{Introduction}

In this paper we prove the following compactness result for holomorphic curves under the splitting of a symplectic manifold along a stable hypersurface. See Section 2 for all the definitions.

Theorem 1.1. Let $(X, \omega, J)$ be a closed symplectic manifold with a tamed almost complex structure. Let $M \subset X$ be a closed stable hypersurface on which all closed Reeb orbits are Morse-Bott non-degenerate. Let $J_{k}$ be the sequence of almost complex structures obtained by stretching the neck along $M$. Let $f_{k}:\left(\Sigma_{k}, j_{k}\right) \rightarrow\left(X, J_{k}\right)$ be a sequence of holomorphic curves of the same genus such that the areas $\int_{\Sigma_{k}} f_{k}^{*} \omega$ are uniformly bounded. Then a subsequence of $\left(f_{k}\right)$ converges to a stable broken holomorphic curve $F$.

Here the notion of convergence includes the following:

- convergence of the underlying Riemann surfaces to a decorated nodal curve; 
- uniform convergence of maps (after reparameterizations) that is smooth outside some collection of simple loops;

- convergence of areas and of homology classes.

This notion of convergence is relevant in symplectic field theory [5]. An analogous compactness theorem was proved by Bourgeois et al. $[\mathbf{3}]$. In fact, Theorem 1.1 is only formulated for a closed manifold $X$ instead of a general symplectic cobordism. This special situation was chosen in order to keep the notation simple and because it naturally arises in applications, e.g., [4]. Moreover, all the essential features are already present in this situation and the proof immediately generalizes to a general symplectic cobordism, see Remark 2.15.

So why bother to present a new proof of the result in $[\mathbf{3}]$ ? In fact, we had developed the ideas for the present paper (in the contact case) before $[3]$ became available. It then became clear that our proof is orthogonal to the one in $[\mathbf{3}]$. Roughly speaking, the proof in $[\mathbf{3}]$ focuses on the domains, ignoring the level structure in the image (and reconstructing it a posteriori), while our proof focuses on the level structure in the image, ignoring the domains (and reconstructing them a posteriori). More technically, the proof in $[\mathbf{3}]$ is based on repeated bubbling off analysis at points where the gradient explodes, while our proof follows the strategy of Gromov's proof [6] in the closed case. Analyzing changes of action and topology of sub-level sets, we determine a priori the levels at which splitting occurs. This reduces the proof to two separate cases: long cylinders of small area and regions with compact image. The first case also arises in $[\mathbf{3}]$ and we quote the main technical result in Section 4. The second case requires the following generalization of Gromov compactness to holomorphic curves with free boundary, which is proved in Section 3 (the precise statement is Theorem 3.2).

Theorem 1.2. Let $f_{k}:\left(\Sigma_{k}, j_{k}\right) \rightarrow(X, J)$ be a sequence of holomorphic curves defined on compact surfaces with boundary of the same genus and with the same number of boundary components. $X$ is not necessarily closed here. Suppose that the areas are uniformly bounded and the images are contained in a compact subset. Moreover, suppose that the boundary circles have disjoint collars whose modulus is uniformly bounded below by a sufficiently large constant and the differentials of $f_{k}$ on these collars are uniformly bounded below and above. Then a subsequence of $\left(f_{k}\right)$ converges to a nodal holomorphic curve.

A similar situation was considered in [11]. The point here is that the convergence is up to the boundary and no area nor topology is lost.

This paper was originally restricted to the case that the hypersurface $M$ is of contact type and separates $X$ into two components. Following the referee's suggestion, we subsequently rewrote the paper to accommodate the general situation of a stable hypersurface considered in [3]. 


\section{Statement of the compactness theorem}

2.1. Hamiltonian structures. Let $M$ be a $(2 n-1)$-dimensional closed manifold. A Hamiltonian structure is a closed 2-form $\omega_{M}$ of (maximal) rank $n-1$ on it. This implies that the kernel $\operatorname{Ker}\left(\omega_{M}\right)=\left\{v \in T_{x} M \mid \omega_{M}(v, \cdot)=\right.$ $0\}$ is a one-dimensional subspace of $T_{x} M$ for all $x \in M$. We will call it the Hamiltonian line field. Such a form $\omega_{M}$ is said to be stable if there exists a 1-form $\lambda$ on $M$ with $\operatorname{Ker}\left(\omega_{M}\right) \subset \operatorname{Ker}(d \lambda)$ while $\left.\lambda\right|_{\operatorname{Ker}\left(\omega_{M}\right)} \neq 0$ everywhere. Notice that the form $\omega_{M}+d(r \lambda)$ is a symplectic form on $(-\varepsilon, \varepsilon) \times M$, with $r \in(-\varepsilon, \varepsilon)$ for $\varepsilon>0$ so small that $r d \lambda+\omega_{M}$ is non-degenerate on $\operatorname{Ker}(\lambda)$ for $|r|<\varepsilon$.

Example 2.1. Let $\lambda \in \Omega^{1}(M)$ be a contact 1-form. Then $d \lambda$ is of maximal rank and thus defines a Hamiltonian structure.

2.2. Reeb vector field. In particular, $\lambda$ uniquely determines a section of the Hamiltonian line field, $R$, by the condition $\lambda(R) \equiv 1$ which we will refer to as the Reeb vector field. A closed orbit $\gamma$ of the Reeb vector field is called non-degenerate if the linearized return map on a transverse section has no eigenvalue 1. The closed Reeb orbits are called Morse-Bott non-degenerate in the sense of $[\mathbf{2}, \mathbf{3}]$ if the following holds: For all $T>0$, the set $\mathcal{N}_{T} \subset M$ formed by the $T$-periodic Reeb orbits is a closed submanifold, the rank of $\left.d \lambda\right|_{\mathcal{N}_{T}}$ is locally constant, and $T_{p} \mathcal{N}_{T}=\operatorname{ker}\left(T_{p} \phi_{T}-\mathbb{1}\right)$ for all $p \in \mathcal{N}_{T}$, where $\phi_{t}$ is the Reeb flow. Note that the case $\operatorname{dim} \mathcal{N}_{T}=1$ corresponds to nondegeneracy of closed Reeb orbits. We will refer to this case as the Morse case.

2.3. Stable hypersurfaces. A hypersurface in a symplectic manifold is called stable if the restriction of the symplectic form is a stable Hamiltonian structure.

Example 2.2. If the Hamiltonian structure of the hypersurface is defined by a contact form $\lambda$, i.e., $\left.\omega\right|_{M}=d \lambda$, we call it a hypersurface of contact type.

The name "stable" is explained by the following.

Lemma 2.3. For a closed hypersurface $M$ in a symplectic manifold $(X, \omega)$, the following are equivalent:

(a) $M$ is stable in the sense of $[\mathbf{1 0}]$, i.e., there exists a tubular neighborhood $(-\varepsilon, \varepsilon) \times M$ of $M=\{0\} \times M$ such that the Hamiltonian line fields on $\{r\} \times M$ are conjugate for all $r \in(-\varepsilon, \varepsilon)$.

(b) There exists a vector field $Y$ transverse to $M$ such that $\operatorname{ker}\left(\left.\omega\right|_{M}\right) \subset$ $\operatorname{ker}\left(\left.L_{Y} \omega\right|_{M}\right)$.

(c) $\left(M, \omega_{M}:=\left.\omega\right|_{M}\right)$ is a stable Hamiltonian structure. 
Proof.

(a) $\Longrightarrow(\mathrm{b})$ : Let $(-\varepsilon, \varepsilon) \times M$ be a tubular neighborhood such that $\operatorname{ker}\left(\left.\omega\right|_{\{r\} \times M}\right)$ is independent of $r$. Set $Y:=\frac{\partial}{\partial r}$ on $(-\varepsilon, \varepsilon) \times M$ and denote by $\phi_{t}:(r, x) \mapsto(r+t, x)$ its flow. Then $\operatorname{ker}\left(\left.\phi_{t}^{*} \omega\right|_{\{0\} \times M}\right)$ is constant in $t$ and differentiation at $t=0$ yields $\operatorname{ker}\left(\left.\omega\right|_{M}\right) \subset \operatorname{ker}\left(\left.L_{Y} \omega\right|_{M}\right)$.

(b) $\Longrightarrow$ (c): Set $\lambda:=\left.i_{Y} \omega\right|_{M}$.

(c) $\Longrightarrow$ (a): The closed 2-form

$$
\tilde{\omega}:=\omega_{M}+d(r \lambda)=\omega_{M}+d r \wedge \lambda+r d \lambda
$$

is symplectic on $(-\varepsilon, \varepsilon) \times M$ for $\varepsilon$ sufficiently small. Its kernel $\operatorname{ker}\left(\left.\tilde{\omega}\right|_{\{r\} \times M}\right)$ contains the Reeb vector field $R$ and is thus independent of $r \in(-\varepsilon, \varepsilon)$. By the coisotropic neighborhood theorem, a neighborhood of $M$ in $(X, \omega)$ is symplectomorphic to $((-\varepsilon, \varepsilon) \times M, \tilde{\omega})$.

2.4. Symplectic cobordisms. A symplectic cobordism is a compact symplectic manifold $(\bar{X}, \omega)$ with stable boundary $\partial \bar{X}=\bar{M} \amalg \underline{M}$. Here we allow one or both of $\bar{M}, \underline{M}$ to be empty. A connected component $M$ of the boundary belongs to $\bar{M}$ if its collar is symplectomorphic to $\left((-\varepsilon, 0] \times M,\left.\omega\right|_{M}+d(r \lambda)\right)$ and to $\underline{M}$ otherwise. Notice that this specification depends on the choice of $\lambda$ and can be reversed by replacing $\lambda$ by $-\lambda$. Let $X$ be the manifold obtained by gluing $\mathbb{R}_{+} \times \bar{M}$ and $\mathbb{R}_{-} \times \underline{M}$ to $\bar{X}$ identifying the corresponding components of the boundary. $X$ is diffeomorphic to $X_{\varepsilon}$ obtained by gluing $[0, \varepsilon) \times \bar{M}$ and $(-\varepsilon, 0] \times M$ to $\bar{X}$ instead of the infinite cylinders. On the collars of the boundary components, we have $\omega=\omega_{M}+d(r \lambda)$. For $\varepsilon$ small enough we can thus extend the symplectic structure to $X_{\varepsilon}$ and obtain a symplectic structure on $X$ which will control the almost complex structure introduced below. We usually refer to $X$ as the symplectic cobordism with positive end $\mathbb{R}_{+} \times \bar{M}$ and negative end $\mathbb{R}_{-} \times \underline{M}$ although the symplectic structure on it is not determined by $\omega$ on $\bar{X}$. We will not need to specify it nor should we due to the character of the control by Hofer's energy. Obvious examples of symplectic cobordisms are closed symplectic manifolds and cylinders over manifolds with stable Hamiltonian structures.

2.5. Almost complex structures. Let $\left(M, \omega_{M}\right)$ be a Hamiltonian structure. Let $J$ be a translation invariant almost complex structure on the cylinder $\mathbb{R} \times M$. Denote by $\lambda \in \Omega^{1}(M)$ the 1 -form which is uniquely determined by $\pi_{M}^{*} \lambda(J(\partial / \partial r)) \equiv 1$ and $\left.\lambda\right|_{\xi}=0$ for the CR-structure $\xi:=T(\{0\} \times M) \cap J(T(\{0\} \times M))$.

We call $J$ tamed by $\omega_{M}$ if

- $J(\partial / \partial r)=R$ is a vector field on $M$;

- $\omega_{M}(R, \cdot)=0$;

- $d \lambda(R, \cdot)=0$

- $\omega_{M}(v, J v)>0$ for all $v \in \xi$. 
Notice that $\lambda(R) \equiv 1$ by the definition of $\lambda$ and the first requirement. The existence of such structure implies that $\omega_{M}$ is stable. Note that there exists an $\varepsilon>0$, such that $\left(\omega_{M}+r d \lambda\right)(v, J v)>0$ for all $v \in \xi$ and $|r| \leq \varepsilon$. There also exists a (possibly smaller) $\varepsilon>0$ such that $\left(\omega_{M}+r d \lambda\right)(v, J v)>0$ for all $v \in T(\mathbb{R} \times M)$ and $|r| \leq \varepsilon$.

An almost complex structure on a symplectic cobordism $(X, \omega)$ is called tamed if it is equal to a structure on the cylinders $\mathbb{R} \times \partial \bar{X}$ tamed by $\left.\omega\right|_{\partial \bar{X}}$ on the cylindrical ends and $\omega(v, J v)>0$ for all $v \in T \bar{X}$. Tamed almost complex structures exist and form a contractible space $\mathcal{J}$ (with the $C^{\infty}$ topology).

2.6. Punctured holomorphic curves. Consider an almost complex structure $J_{M}$ on $\mathbb{R} \times M$ which is tamed by a stable Hamiltonian structure $\omega_{M}$. Let $\lambda$ be the corresponding 1-form. Let $\gamma:[0, T] \rightarrow M$ be a (not necessarily simple) closed orbit of the Reeb vector field $R$ of period $T$. A $J$-holomorphic map $f=(a, u): D \backslash 0 \rightarrow \mathbb{R} \times M$ of the punctured unit disk is called positively (resp. negatively) asymptotic to $\gamma$ if $\lim _{\rho \rightarrow 0} a\left(\rho e^{i \theta}\right)=\infty$ (resp. $-\infty$ ) and $\lim _{\rho \rightarrow 0} u\left(\rho e^{i \theta}\right)=\gamma(T \theta / 2 \pi)\left(\right.$ resp. $\left.\lim _{\rho \rightarrow 0} u\left(\rho e^{i \theta}\right)=\gamma(-T \theta / 2 \pi)\right)$ uniformly in $\theta$.

Now let $(X, \omega)$ be a symplectic cobordism with tamed almost complex structure $J$. A punctured holomorphic curve in $(X, J)$ consists of the following data:

- A Riemann surface $(\Sigma, j)$ with distinct positive and negative points $\bar{z}:=\left(\bar{z}_{1}, \ldots, \bar{z}_{\bar{p}}\right), \underline{z}:=\left(\underline{z}_{1}, \ldots, \underline{z}_{p}\right)$. We denote by $\Sigma^{*}:=\Sigma \backslash\left\{\bar{z}_{i}, \underline{z}_{j}\right\}$ the corresponding punctured Riemann surface.

- Corresponding vectors $\bar{\Gamma}:=\left(\bar{\gamma}_{1}, \ldots, \bar{\gamma}_{\bar{p}}\right), \underline{\Gamma}:=\left(\underline{\gamma}_{1}, \ldots, \underline{\gamma}_{p}\right)$ of closed Reeb orbits in $\bar{M}, \underline{M}$.

- A $(j, J)$-holomorphic map $f: \Sigma^{*} \rightarrow X$ which is positively (resp. negatively) asymptotic to $\bar{\gamma}_{j}$ (resp. $\underline{\gamma}_{j}$ ) at the punctures $\bar{z}_{j}$ (resp. $\underline{z}_{j}$ ).

In a symplectization $\mathbb{R} \times M$, a cylinder over a $T$-periodic Reeb orbit $\gamma$

$$
f: \mathbb{R} \times \frac{\mathbb{R}}{\mathbb{Z}} \rightarrow \mathbb{R} \times M, \quad(s, t) \mapsto(T s, \gamma(T t))
$$

is a punctured holomorphic curve with one positive and one negative puncture.

Denote by $\bar{\Sigma}$ the compactification of the punctured surface $\Sigma^{*}$ by adding a circle at each puncture, and abusing notation by $\bar{X}$ the compactification of the manifold $X$ by adding a copy of $\bar{M}$ resp. $\underline{M}$ at the positive resp. negative end. The latter space is indeed diffeomorphic to the original symplectic manifold with boundary. In view of the behavior near the punctures, the holomorphic map $f: \Sigma^{*} \rightarrow X$ above extends to a continuous map $\bar{f}: \bar{\Sigma} \rightarrow \bar{X}$. This extension represents a relative homology class

$$
[\bar{f}] \in H_{2}(\bar{X}, \bar{\Gamma} \cup \underline{\Gamma}) .
$$


2.7. Neck stretching. Consider a closed connected symplectic manifold $(X, \omega)$ and a closed stable hypersurface $M \subset X$. Let $\lambda$ be the corresponding 1-form on $M$ and $\omega_{M}:=\left.\omega\right|_{M}$. Fix a parameterization of a bi-collar neighborhood by $[-\varepsilon, \varepsilon] \times M$ on which $\omega=\omega_{M}+d(r \lambda)$. Pick a tamed almost complex structure $J$ on $(X, \omega)$, i.e., $\omega(X, J X)>0$ for any $X \in T X$. Suppose its restriction $J_{M}$ to $[-\varepsilon, \varepsilon] \times M$ is the restriction of an $\omega_{M}$-tamed structure on $\mathbb{R} \times M$ with $\lambda_{J}=\lambda$.

Define a sequence of symplectic manifolds as follows. Denote by $\bar{X}_{0}:=$ $X \backslash(-\varepsilon, \varepsilon) \times M$ the manifold with boundary $\partial \bar{X}_{0}=M_{+} \amalg M_{-}$, where $M_{-}:=$ $\{-\varepsilon\} \times M$ and $M_{+}:=\{\varepsilon\} \times M$. For an integer $k \geq 0$, let

$$
X_{k}:=\bar{X}_{0} \cup_{M_{-} \amalg M_{+}}([-k-\varepsilon, \varepsilon] \times M)
$$

where $M_{-}$is identified with $\{-k-\varepsilon\} \times M$ while $M_{+}$is identified with $\{\varepsilon\} \times M$. This manifold is, of course, canonically diffeomorphic to $X$, but $X_{k}$ is a more convenient domain for describing the deformed structures. Define an almost complex structure on $X_{k}$ by

$$
J_{k}:= \begin{cases}J & \text { on } \bar{X}_{0}, \\ J_{M} & \text { on }[-k-\varepsilon, \varepsilon] \times M .\end{cases}
$$

This almost complex structure is tamed by the symplectic form on $X_{k}$ given by

$$
\omega_{\phi}:= \begin{cases}\omega & \text { on } \bar{X}_{0}, \\ \omega_{M}+d(\phi \lambda) & \text { on }[-k-\varepsilon, \varepsilon] \times M,\end{cases}
$$

where $\phi:[-k-\varepsilon, \varepsilon] \rightarrow[-\varepsilon, \varepsilon]$ is a diffeomorphism with $\phi^{\prime} \equiv 1$ near the end points of the interval. Note that the cohomology class $\left[\omega_{\phi}\right]$ does not depend on the choice of such a function $\phi$. As $k \rightarrow \infty$, an observer in $X_{k}$ will see either one of the following symplectic cobordisms arising as a limit: one of the connected components of $X_{0}\left(\bar{X}_{0}\right.$ completed with half cylinders over $M$ at their boundary components) or $\mathbb{R} \times M$. The almost complex structures will converge to tamed ones on all of these cobordisms, $\omega_{M}$-tamed on the ends and translation invariant on $\mathbb{R} \times M$. The symplectic forms, however, will only converge in a certain sense if we carefully choose a sequence $\left(\phi_{k}\right)$ of such functions. Moreover, there is no canonical choice of symplectic forms in the limit.

Example 2.4 [The contact case]. Let us consider the case that $M \subset X$ is a hypersurface of contact type and that $M$ separates $X$ into two components $\bar{X}_{0}=\bar{X}_{0}^{+} \amalg \bar{X}_{0}^{-}$. In this case, we can define another sequence of symplectic structures $\omega_{k}$ by setting

$$
\omega_{k}:= \begin{cases}\omega & \text { on } \bar{X}_{0}^{+}, \\ d\left(e^{r} \lambda\right) & \text { on }[-k, 0] \times M, \\ e^{-k} \omega & \text { on } \bar{X}_{0}^{-}\end{cases}
$$


Here we use a slightly different symplectic identification of a neighborhood of $M$ with $\left([\log (1-\varepsilon), \log (1+\varepsilon)] \times M, d\left(e^{r} \lambda\right)\right)$. The results of the present paper were originally restricted to this case. This explains why all our figures show this feature.

In contrast to the general case, here the limits are equipped with natural symplectic structures: Each cylinder $\mathbb{R} \times M$ is equipped with the symplectic form $d\left(e^{r} \lambda\right)$, and $X_{0}=X_{0}^{+} \amalg X_{0}^{-}$is equipped with the symplectic forms $\omega^{ \pm}$on $X_{0}^{ \pm}$satisfying $\omega^{ \pm}=\omega$ on $\bar{X}_{0}^{ \pm}$and $\omega^{ \pm}=d\left(e^{r} \lambda\right)$ on $\mathbb{R}_{ \pm} \times M$. Notice that to "see" these symplectic structures on $\mathbb{R} \times M$ and $X_{0}^{-}$in the limit, the observer has to rescale them. For example, $\omega^{-}$is obtained as the limit of $e^{k} \omega_{k}$ on compact subsets of $X_{0}^{-}$.

2.8. Broken holomorphic curves. We will now describe the limiting objects of sequences of $J_{k}$-holomorphic curves in the neck stretching procedure. We retain the setup of the preceding section. Assume for simplicity that $M$ is connected; see Remark 2.14 at the end of this section for the modifications required in the disconnected case.

Given an integer $N \geq 1$, we set

$$
\left(X^{(\nu)}, J^{(\nu)}\right):= \begin{cases}\left(X_{0}, J\right) & \text { for } \nu=0, N+1 \\ \left(\mathbb{R} \times M, J_{M}\right) & \text { for } \nu=1, \ldots, N .\end{cases}
$$

Define the disjoint union

$$
X^{*}:=X^{(0)} \amalg \coprod_{\nu=1}^{N} X^{(\nu)},
$$

equipped with the almost complex structures $J^{*}$ induced by the $J^{(\nu)}$. Glue the positive boundary component of the compactification $\overline{X^{(\nu)}}$ (by copies of $M)$ to the negative boundary component of $\overline{X^{(\nu+1)}}$ to obtain a compact topological space $\bar{X}$.

Note that $\bar{X}$ is naturally homeomorphic to $X$ (see the proof of Lemma 2.6 for a particular homeomorphism), so we can identify homology classes in $X$ and $\bar{X}$. The natural inclusion $X^{*} \subset \bar{X}$ is dense. Its complement consists of a finite number of disjoint copies of $M$.

Let $\bar{\Sigma}$ be a closed oriented surface, $\mathbf{z}=\left(z^{1}, \ldots, z^{q}\right) \in \bar{\Sigma} q$ distinct points, and $\Delta=\Delta_{n} \cup \Delta_{p} \subset \bar{\Sigma} \backslash\left\{z^{1}, \ldots, z^{q}\right\}$ a collection of finitely many disjoint simple loops divided into two disjoint sets. Denote by $\Sigma$ the surface obtained by collapsing the curves in $\Delta_{n}$ to points. This way we think of $\Delta_{n}$ as the set of nodes of $\Sigma$. The points $z^{1}, \ldots, z^{q}$ will usually be referred to as marked points. Write

$$
\Sigma^{*}:=\Sigma \backslash \Delta_{p}=: \Sigma^{(0)} \amalg \coprod_{\nu=1}^{N} \Sigma^{(\nu)},
$$


as a disjoint union of (not necessarily connected) components $\Sigma^{(\nu)}$. Let $j$ be a conformal structure on $\bar{\Sigma} \backslash \Delta$ such that $(\bar{\Sigma} \backslash \Delta, j)$ is a punctured Riemann surface together with an identification of distinct pairs of punctures given by the elements of $\Delta$. This gives $\Sigma^{*}$ the structure of a nodal punctured Riemann surface with a remaining identification of punctures given by the loops $\left\{\delta^{i}\right\}_{i \in I}=\Delta_{p}$. A broken holomorphic curve (with $N+1$ levels)

$$
F=\left(F^{(0)}, F^{(1)}, \ldots, F^{(N)}\right):\left(\Sigma^{*}, j\right) \rightarrow\left(X^{*}, J^{*}\right)
$$

is a collection of punctured holomorphic curves $F^{(\nu)}:\left(\Sigma^{(\nu)}, j\right) \rightarrow\left(X^{(\nu)}, J^{(\nu)}\right)$ such that $F: \Sigma^{*} \rightarrow X^{*}$ extends to a continuous map $\bar{F}: \Sigma \rightarrow \bar{X}$. Moreover, we require that $F$ is stable: For $1 \leq \nu \leq N, F^{(\nu)}$ is not the union of cylinders over closed Reeb orbits without any marked points on them. Moreover, $\Sigma^{*}$ does not contain any sphere with less than three special points (punctures, nodal or marked points), nor a torus without special points, on which $F$ is constant.

See Figure 1 for the case $N=2$ and $X_{0}=X_{0}^{+} \amalg X_{0}^{-}$consisting of two connected components $X^{+}$and $X^{-}$. In this case, we denote by $X_{ \pm}^{(0)}, F_{ \pm}^{(0)}$ the sets and maps which belong to either side of $M$.

Note that, by continuity of $\bar{F}$, the number of positive punctures of $F^{(\nu)}$ agrees with the number of negative punctures of $F^{(\nu+1)}$ and the asymptotic Reeb orbits at the punctures agree correspondingly: $\bar{\Gamma}^{(\nu)}=\underline{\Gamma}^{(\nu+1)}$.

Remark 2.5. Every broken holomorphic curve has an underlying graph. Its vertices are the connected components of $\Sigma^{*}$, and each loop $\delta^{i}$ defines an edge between the corresponding components. Note that if $\bar{\Sigma}$ has genus zero, the underlying graph is a tree. See Figure 1 for a genus three case.

Pick a homeomorphism $\Phi: \bar{X} \rightarrow X$ which is the identity on $X \backslash(-\varepsilon, \varepsilon) \times M$ and of the form $(r, x) \in \mathbb{R} \times M \mapsto(\phi(r), x)$ on the cylindrical parts, where the homeomorphism

$$
\phi:[-\varepsilon, \infty] \cup_{ \pm \infty}[-\infty, \infty] \cup_{ \pm \infty} \cdots \cup_{ \pm \infty}[-\infty, \varepsilon] \rightarrow[-\varepsilon, \varepsilon]
$$

is a diffeomorphism outside the points $\pm \infty$ and $\phi(r)=r$ near $\pm \varepsilon$. Denote by $\phi^{(\nu)}$ the restriction to the union of the first and last interval if $\nu=0$ and to the $(\nu+1)$ st interval if $\nu=1, \ldots, N$. Note that $\Phi^{*} \omega$ defines a symplectic form $\omega_{\phi}$ on $X^{*}$ which is of the form $\omega_{M}+d(\phi \lambda)$ on the cylindrical part. Moreover, the continuous map $\Phi \circ \bar{F}: \Sigma \rightarrow X$ represents the homology class $A \in H_{2}(X ; \mathbb{Z})$.

Lemma 2.6. The homology class $A:=[\bar{F}] \in H_{2}(X ; \mathbb{Z})$ of a non-constant broken holomorphic curve $F:\left(\Sigma^{*}, j\right) \rightarrow\left(X^{*}, J^{*}\right)$ satisfies

$$
\langle[\omega], A\rangle=\int_{\Sigma^{*}}(\Phi \circ F)^{*} \omega=\int_{\Sigma^{*}} F^{*} \omega_{\phi}>0 .
$$

In particular, the integral of $\omega_{\phi}$ does not depend on the choice of $\phi$. 


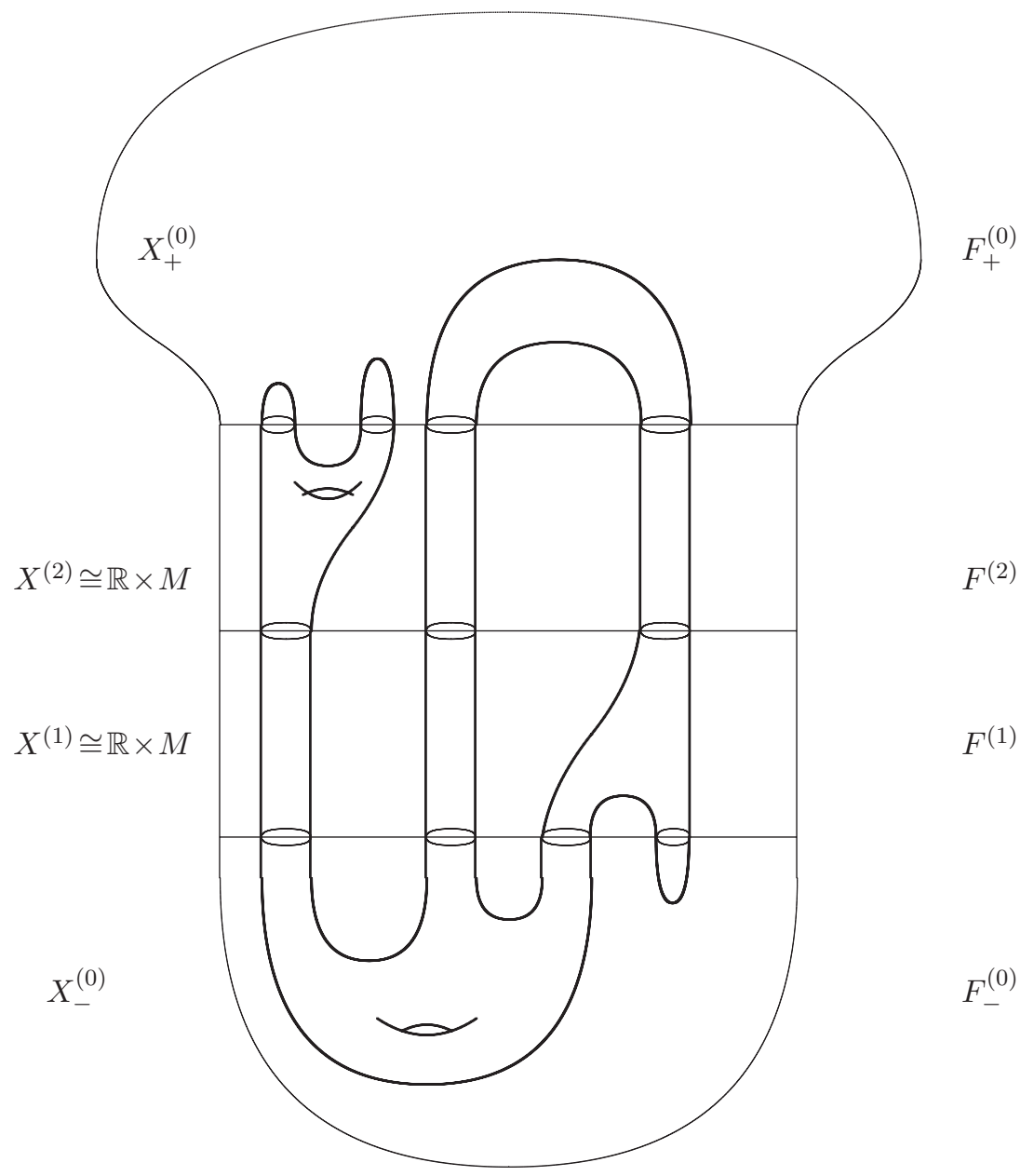

Figure 1. A broken holomorphic curve of genus 3 in the setting $X_{0}=X_{0}^{+} \amalg X_{0}^{-}$.

Proof. We first note that $\Phi^{*} \omega$ is tamed by $J^{*}$. Hence

$$
\int_{\Sigma^{*}}(\Phi \circ F)^{*} \omega=\int_{\Sigma^{*}} F^{*} \omega_{\phi}>0
$$

for every non-constant broken holomorphic curve $F:\left(\Sigma^{*}, j\right) \rightarrow\left(X^{*}, J^{*}\right)$.

For the proof of the equality, we first describe a situation were Stokes' theorem holds which is maybe well-known but included for sake of completeness.

2.9. Claim. Let $F: C \rightarrow M$ be a continuous map from a smooth compact manifold $C$ with boundary $\partial C=B$ to another smooth manifold $M$. Suppose that $\left.F\right|_{\text {int } C}$ and $\left.F\right|_{B}$ are smooth and there exists an exhaustion $C_{1} \subset C_{2} \subset$ 
$\cdots \subset \operatorname{int} C$, where $C_{i}$ are smooth manifolds with boundaries $B_{i}$, together with diffeomorphisms $\varphi_{i}: B \rightarrow B_{i} \subset C$ such that $\left.F \circ \varphi_{i} \rightarrow F\right|_{B}$ in $C^{1}$. Then Stokes' theorem holds, i.e., for any $k$-form $\lambda$ on $M, k=\operatorname{dim} C-1$, we have

$$
\int_{\operatorname{int} C} d\left(F^{*} \lambda\right)=\int_{B} F^{*} \lambda
$$

whenever the integrand of the left-hand side is absolutely integrable.

Using the standard form of Stokes' theorem the proof is very simple. Since the $C_{i}$ are smooth manifolds, we have

$$
\int_{C_{i}} F^{*} d \lambda=\int_{B_{i}} F^{*} \lambda
$$

Now by assumption, both sides converge to the corresponding expressions of the claim.

Turning back to the proof of the lemma, note that in view of the asymptotic behavior of the $F^{(\nu)}$ the assumptions of the claim are satisfied. Moreover, in some small neighborhood $N^{(\nu)}$ of the corresponding set of Reeb orbits $\bar{\Gamma}^{(\nu)}=\underline{\Gamma}^{(\nu+1)}$, the form $\omega_{M}$ is exact, i.e., there is a 1-form $\alpha^{(\nu)}$ on $N^{(\nu)}$ with $d \alpha^{(\nu)}=\left.\omega_{M}\right|_{N^{(\nu)}}$. For $R$ sufficiently large $\bar{\Gamma}_{R}^{(\nu)}:=F^{(\nu)}\left(\Sigma^{(\nu)}\right) \cap(\{R\} \times$ $M) \subset N^{(\nu)}$ consists of as many loops as $\bar{\Gamma}^{(\nu)}$ and each of them is $C^{1}$ close to the corresponding Reeb orbit. Analogously, $\underline{\Gamma}_{R}^{(\nu)}:=F^{(\nu)}\left(\Sigma^{(\nu)}\right) \cap$ $(\{-R\} \times M) \subset N^{(\nu-1)}$ is a set of loops which is $C^{1}$-close to $\underline{\Gamma}^{(\nu)}$. Now $\bar{\gamma}_{R}^{(\nu)}:=\left(F^{(\nu)}\right)^{-1}\left(\bar{\Gamma}_{R}^{(\nu)}\right)$ and $\underline{\gamma}_{R}^{(\nu)}:=\left(F^{(\nu)}\right)^{-1}\left(\underline{\Gamma}_{R}^{(\nu)}\right)$ are small simple loops around the corresponding positive and negative punctures, respectively. The region between $\bar{\gamma}_{R}^{(\nu)}$ and $\underline{\gamma}_{R}^{(\nu+1)}$ in $\bar{\Sigma}$ consists of a disjoint union $A^{(\nu)}$ of annuli bounded by one component of each set. So we can deform $\bar{F}$ to a smooth map $\tilde{F}: \Sigma \rightarrow \bar{X}$ (i.e., $\Phi \circ \tilde{F}$ is smooth) which agrees with $F$ outside these annuli and maps each $A^{(\nu)}$ into the corresponding neighborhood $N^{(\nu)}$. Now by the claim above,

$$
\begin{aligned}
\int_{A^{(\nu)}} F^{*}\left(\omega_{M}+d(\phi \lambda)\right)= & \int_{\underline{\gamma}_{R}^{(\nu+1)}}\left(F^{(\nu+1)}\right)^{*}\left(\left(\alpha^{(\nu)}+\phi^{(\nu+1)}(-R) \lambda\right)\right. \\
& \left.-\int_{\bar{\gamma}_{R}^{(\nu)}}\left(F^{(\nu)}\right)^{*}\left(\alpha^{(\nu)}+\phi^{(\nu)}(R) \lambda\right)\right),
\end{aligned}
$$

since the integrals over the common boundaries of the annuli cancel each other. But the integrals on the right-hand side are exactly the same as the integral of $\tilde{F}^{*}\left(\alpha^{(\nu)}+\phi \lambda\right)$ over the same set of loops. Thus by (ordinary) Stokes' theorem, we obtain

$$
\int_{A^{(\nu)}} F^{*}\left(\omega_{M}+d(\phi \lambda)\right)=\int_{A^{(\nu)}} \tilde{F}^{*}\left(\omega_{M}+d(\phi \lambda)\right) .
$$


Since $F=\tilde{F}$ outside the annuli $A^{(\nu)}$, we conclude that

$$
\int_{\Sigma^{*}} F^{*} \Phi^{*}(\omega)=\int_{\bar{\Sigma}} \tilde{F}^{*} \Phi^{*}(\omega)=\langle[\omega],[\tilde{F}]\rangle=\langle[\omega], A\rangle,
$$

where $[\tilde{F}]=[\bar{F}]=A$ because $\tilde{F}$ was a deformation of $\bar{F}$.

For $\Delta_{p}=\left(\delta^{i}\right)_{i \in I}$, let $I^{(\nu)}$ be the set of $i$ such that $\delta^{i}$ is adjacent to $\Sigma^{(\nu)}$ and $\Sigma^{(\nu+1)}$. Fix disjoint embedded annuli

$$
A^{i} \cong[-1,1] \times \frac{\mathbb{R}}{\mathbb{Z}} \subset \Sigma \backslash \Delta_{n}
$$

such that $\{0\} \times \mathbb{R} / \mathbb{Z}=\delta^{i}$, and $\{-1\} \times \mathbb{R} / \mathbb{Z} \subset \Sigma^{(\nu)}$ for $i \in I^{(\nu)}$.

2.10. Convergence to a stable broken holomorphic curve. Now we return to the sequence of almost complex manifolds $\left(X_{k}, J_{k}\right)$ defined above. For $R \in \mathbb{R}$, denote by

$$
X_{k} \rightarrow \bar{X}_{0} \cup_{M_{-} \amalg M_{+}}[-k-\varepsilon+R, \varepsilon+R] \times M, \quad x \mapsto x+R
$$

the map which equals the identity on $\bar{X}_{0}$ and is given by $(r, x) \mapsto(r+R, x)$ on $[-\varepsilon-k, \varepsilon] \times M$. Consider sequences of numbers

$$
-k=r_{k}^{(0)}<r_{k}^{(1)}<\cdots<r_{k}^{(N+1)}=0
$$

such that $r_{k}^{(\nu+1)}-r_{k}^{(\nu)} \rightarrow \infty$ as $k \rightarrow \infty$. Define the manifolds

$$
\begin{aligned}
X_{k}^{(\nu)} & :=\bar{X}_{0} \cup_{M_{-} \amalg M_{+}}\left[-\varepsilon-k-r_{k}^{(\nu)}, \varepsilon-r_{k}^{(\nu)}\right] \times M, \quad \nu=1, \ldots, N, \\
X_{k}^{(0)} & :=\left[-\frac{k}{2}, \varepsilon\right] \times M \cup_{M_{+}} \bar{X}_{0} \cup_{M_{-}}\left[-\varepsilon, \frac{k}{2}\right] \times \frac{M}{(-k / 2, x)} \sim\left(\frac{k}{2}, x\right) .
\end{aligned}
$$

Note that for an observer in $(0, x) \in X_{k}^{(\nu)}$ with $x \in M$, we have $X_{k}^{(\nu)} \rightarrow X^{(\nu)}$ on compact subsets containing $(0, x)$ as $k \rightarrow \infty$. For maps $f_{k}: \Sigma_{k} \rightarrow X_{k}$ define

$$
f_{k}^{(\nu)}: \Sigma_{k} \rightarrow X_{k}^{(\nu)}, \quad f_{k}^{(\nu)}(z):=f_{k}(z)-r_{k}^{(\nu)}, \quad \nu=1, \ldots, N .
$$

For $\nu=0$, let $f_{k}^{(0)}: \Sigma_{k} \rightarrow X_{k}^{(0)}$ be composition of $f$ with the map $X_{k} \rightarrow X_{k}^{(0)}$ that equals the identity on $[-k / 2, \varepsilon] \times M \cup \bar{X}_{0}$ and maps $(r, x) \mapsto(r+k, x)$ on $[-k-\varepsilon,-k / 2] \times M$. Denote by $\pi_{\mathbb{R}}: \mathbb{R} \times M \rightarrow \mathbb{R}$ and $\pi_{M}: \mathbb{R} \times M \rightarrow M$ the obvious projections.

Finally, let a sequence of diffeomorphisms $\phi_{k}:[-\varepsilon-k, \varepsilon] \rightarrow[-\varepsilon, \varepsilon]$ be given with $\phi_{k}^{\prime} \equiv 1$ near the boundaries. Define

$$
\phi_{k}^{(\nu)}:=\phi_{k}\left(\cdot+r_{k}^{(\nu)}\right):\left[-\varepsilon-k-r_{k}^{(\nu)}, \varepsilon-r_{k}^{(\nu)}\right] \rightarrow[-\varepsilon, \varepsilon]
$$

for $\nu=1, \ldots, N$. For $\nu=0$, let

$$
\phi_{k}^{(0)}:[-k / 2, \varepsilon] \cup \frac{[-\varepsilon, k / 2]}{(-k / 2 \sim k / 2)} \longrightarrow[-\varepsilon, \varepsilon]
$$


be the map which equals $\phi_{k}$ on $[-k / 2, \varepsilon]$ and $\phi_{k}(\cdot-k)$ on $[-\varepsilon, k / 2]$. Suppose that $\lim _{k \rightarrow \infty} \phi_{k}^{(\nu)}=\phi^{(\nu)}$ in $C_{\text {loc }}^{\infty}$ for $\nu=0, \ldots, N$, with $\phi^{(\nu)}$ as defined right before Lemma 2.6.

Definition 2.7. A sequence of punctured holomorphic curves with $q$ marked points $f_{k}:\left(\Sigma_{k}, j_{k}, \mathbf{z}_{k}\right) \rightarrow\left(X_{k}, J_{k}\right)$ converges to a broken holomorphic curve with $q$ marked points $F:\left(\Sigma^{*}, j, \mathbf{z}\right) \rightarrow\left(X^{*}, J^{*}\right)$ if there exist orientation preserving diffeomorphisms $\varphi_{k}: \Sigma_{k} \rightarrow \bar{\Sigma}$ and numbers $-k=r_{k}^{(0)}<r_{k}^{(1)}<$ $\cdots<r_{k}^{(N+1)}=0$ with $r_{k}^{(\nu+1)}-r_{k}^{(\nu)} \rightarrow \infty$ such that the following holds:

(a) $\left(\varphi_{k}\right)_{*} j_{k} \rightarrow j$ in $C_{\mathrm{loc}}^{\infty}$ on $\Sigma^{*} \backslash \Delta_{n}$ and $\varphi_{k}\left(z_{k}^{l}\right) \rightarrow z^{l}$ for $l=1, \ldots, q$.

(b) For every $i \in I$, the annulus $\left(A^{i},\left(\varphi_{k}\right)_{*} j_{k}\right)$ is conformally equivalent to a standard annulus $\left[-L_{k}^{i}, L_{k}^{i}\right] \times \mathbb{R} / \mathbb{Z}$ by a diffeomorphism of the form $(s, t) \mapsto(\sigma(s), t)$, with $L_{k}^{i} \rightarrow \infty$ as $k \rightarrow \infty$.

(c) $f_{k}^{(\nu)} \circ \varphi_{k}^{-1} \rightarrow F^{(\nu)}$ in $C_{\mathrm{loc}}^{\infty}$ on $\Sigma^{(\nu)} \backslash \Delta_{n}$ and in $C_{\mathrm{loc}}^{0}$ on $\Sigma^{(\nu)}$.

(d) For every $i \in I^{(\nu)}, \pi_{M} \circ f_{k} \circ \varphi_{k}^{-1} \rightarrow \pi_{M} \circ \bar{F}$ uniformly on $A^{i}$. Moreover, for every $R>0$, there exist $\rho>0$ and $K \in \mathbb{N}$ such that $\pi_{\mathbb{R}} \circ f_{k} \circ$ $\varphi_{k}^{-1}(s, t) \in\left[r_{k}^{(\nu)}+R, r_{k}^{(\nu+1)}-R\right]$ for all $k \geq K$ and all $(s, t) \in A^{i}$ with $|s| \leq \rho$.

(e) $\int_{\Sigma_{k}} f_{k}^{*} \omega_{\phi_{k}} \rightarrow \int_{\Sigma^{*}} F^{*} \omega_{\phi}=\omega([\bar{F}])$.

\section{Remark 2.8.}

(1) Condition (b) implies that the conformal structures $\left(\varphi_{k}\right)_{*} j_{k}$ converge to $j$ as a (partially) decorated nodal surface in the sense of [3].

(2) The sequence $r_{k}^{(\nu)}$ is almost uniquely determined by condition (c): If condition (c) also holds with $r_{k}^{(\nu)}$ replaced by $s_{k}^{(\nu)}$, then $r_{k}^{(\nu)}-s_{k}^{(\nu)}$ remains bounded as $k \rightarrow \infty$. Here we use the stability assumption.

(3) Condition (d) implies convergence of homology classes $\left[f_{k} \circ \varphi_{k}^{-1}\right] \rightarrow$ $[\bar{F}] \in H_{2}(X ; \mathbb{Z})$ under the canonical identifications $H_{2}\left(X_{k}\right) \cong H_{2}(\bar{X}) \cong$ $\mathrm{H}_{2}(X)$.

2.11. Compactness. We will actually prove the following more precise version of Theorem 1.1 .

Theorem 2.9 (Gromov-Hofer compactness). Let $(X, \omega)$ be a closed symplectic manifold and $M \subset X$ a stable closed hypersurface. Assume that all closed Reeb orbits on $(M, \lambda)$ are Bott non-degenerate. Define $\left(X_{k}, J_{k}\right)$ as above. Let $f_{k}:\left(\Sigma_{k}, j_{k}, \mathbf{z}_{k}\right) \rightarrow X_{k}$ be a sequence of $J_{k}$-holomorphic curves of the same genus with $q$ marked points and with uniformly bounded area $\int_{\Sigma_{k}} f_{k}^{*} \omega \leq E_{0}$, where we identify $X_{k} \cong X$. Then a subsequence of $\left(f_{k}\right)$ converges to a broken holomorphic curve with $q$ marked points $F:\left(\Sigma^{*}, j, \mathbf{z}\right) \rightarrow\left(X^{*}, J^{*}\right)$ in the sense of Definition 2.7 .

The proof of Theorem 2.9 is carried out in Section 5. As explained above, the proof reduces to two separate cases: long cylinders of small area and 
regions with compact image. The first case also arises in $[\mathbf{3}]$ and we quote the main technical result in Section 4. The second case requires a generalization of Gromov compactness to holomorphic curves with free boundary, which is proved in Section 3.

The following corollary is sometimes useful [4].

Corollary 2.10. In the situation of Theorem 2.9, assume in addition that the genus is zero and all the $f_{k}$ represent a homology class $A$ which cannot be written as $A=B+C$ with $B, C \in H_{2}(X ; \mathbb{Z})$ satisfying $\omega(B), \omega(C)>0$. Then $F$ is a broken holomorphic curve without nodes, i.e., $\Delta_{n}=\emptyset$ and the convergence statements simplify accordingly .

Proof. Suppose $F$ has a node. Since the genus is zero, the node decomposes the domain $\Sigma$ into two connected components $\Sigma_{0}, \Sigma_{1}$. The restrictions of $F$ to these components define non-constant stable broken holomorphic curves $F_{0}, F_{1}$ representing homology classes $A_{0}, A_{1} \in H_{2}(X ; \mathbb{Z})$ with $A_{0}+A_{1}=A$. Then Lemma 2.6 yields $\omega\left(A_{0}\right), \omega\left(A_{1}\right)>0$, contradicting the assumption on $A$.

Next consider the case that $M \subset X$ is a hypersurface of contact type which separates $X$ into $\bar{X}_{0}=\bar{X}_{0}^{+} \amalg \bar{X}_{0}^{-}$. Define the symplectic structures $\omega_{k}$ on $X_{k}$ and $\omega^{ \pm}$on $X_{0}^{ \pm}$as in Example 2.4. Denote by

$$
F_{ \pm}^{(0)}: \Sigma_{ \pm}^{(0)} \rightarrow X_{0}^{ \pm} \subset X_{0}
$$

the components of $F^{(0)}$ in $X_{0}^{ \pm}$. The following corollary is important for the applications in [4]. It will be proved at the end of Section 5 .

Corollary 2.11. In the situation of Theorem 2.9, assume in addition that $M$ is of contact type and separates $X$ into two components $X_{0}^{ \pm}$. Then with the above notation, we have

$$
\lim _{k \rightarrow \infty} \int_{\Sigma_{k}} f_{k}^{*} \omega_{k}=\int_{\Sigma^{(0)}}\left(F_{+}^{(0)}\right)^{*} \omega^{+} .
$$

2.12. Reformulation. Before turning to the proof, let us give a reformulation of Theorem 2.9. Define the intervals

$$
E^{(\nu)}:= \begin{cases}{[0, \infty)} & \text { for } \nu=0, \\ (-\infty, \infty) & \text { for } \nu=1, \ldots, N, \\ (-\infty, 0] & \text { for } \nu=N+1\end{cases}
$$

and glue their compactifications along their endpoints to

$$
\bar{E}:=\overline{E^{(1)}} \cup_{ \pm \infty} \overline{E^{(2)}} \cup_{ \pm \infty} \cdots \cup_{ \pm \infty} \overline{E^{(N+1)}} .
$$

Then the topological space $\bar{X}$ can be written as

$$
\bar{X}=\bar{X}_{0} \cup_{M_{-} \amalg M_{+}}(\bar{E} \times M) .
$$


Since $\bar{E}$ is homeomorphic to a closed interval, $\bar{X}$ has naturally the structure of a topological manifold. We can define a smooth structure on $\bar{X}$ as follows. Set $E^{*}:=\coprod_{\nu=1}^{N} E^{(\nu)} \subset \bar{E}$. Pick a strictly increasing smooth parameterization

$$
\Phi: \bar{E} \rightarrow[a, b]
$$

onto a closed interval $[a, b] \subset \mathbb{R}$ whose restriction to $E^{*}$ is a diffeomorphism onto its image. The induced homeomorphism $\hat{\Phi}: \bar{X} \rightarrow Y$ onto the smooth manifold

$$
Y:=\bar{X}_{0} \cup_{M_{-} \amalg M_{+}}[a, b] \times M
$$

(with the identity on $\bar{X}_{0}$ ) induces a smooth structure on $\bar{X}$. Any two such smooth structures are obviously diffeomorphic. Fix a model $Y$ and denote the restrictions of $\Phi$ to $E^{(\nu)}$ by

$$
\begin{gathered}
\Phi^{(0)}:[0, \infty) \rightarrow\left[a^{(0)}, a^{(1)}\right), \quad \Phi^{(N+1)}:(-\infty, 0] \rightarrow\left(a^{(N)}, a^{(N+1)}\right], \\
\Phi^{(\nu)}:(-\infty, \infty) \rightarrow\left(a^{(\nu-1)}, a^{(\nu)}\right), \quad \nu=1, \ldots, N,
\end{gathered}
$$

where $a=a^{(0)}<a^{(1)}<\cdots<a^{(N+1)}=b$.

Now consider sequences $-k=r_{k}^{(0)}<r_{k}^{(2)}<\cdots<r_{k}^{(N+1)}=0$ with $r_{k}^{(\nu+1)}-r_{k}^{(\nu)} \rightarrow \infty$ as above. For diffeomorphisms $\Phi_{k}:[-k, 0] \rightarrow[a, b]$ define

$$
\Phi_{k}^{(\nu)}:\left[-k-r_{k}^{(\nu)},-r_{k}^{(\nu)}\right] \rightarrow[a, b], \quad \Phi_{k}^{(\nu)}(x):=\Phi_{k}\left(x+r_{k}^{(\nu)}\right) .
$$

We impose the following hypothesis on the sequence $\left(\Phi_{k}\right)$ :

$$
\Phi_{k}^{(\nu)} \underset{k \rightarrow \infty}{\longrightarrow} \Phi^{(\nu)}
$$

in $C_{\mathrm{loc}}^{\infty}$ on $E^{(\nu)}$. Note that the convergence makes sense because, by the hypothesis $r_{k}^{(\nu+1)}-r_{k}^{(\nu)} \rightarrow \infty$, every compact subset of $E^{(\nu)}$ is contained in the domain of definition of $\Phi_{k}^{(\nu)}$ for $k$ sufficiently large. Denote by $\hat{\Phi}_{k}: X_{k} \rightarrow Y$ the map induced by $\Phi_{k}$ and the identity on $\bar{X}_{0}$.

Proposition 2.12. Let $f_{k}:\left(\Sigma_{k}, j_{k}\right) \rightarrow\left(X_{k}, J_{k}\right)$ be a sequence of punctured holomorphic curves and $F:\left(\Sigma^{*}, j\right) \rightarrow\left(X^{*}, J^{*}\right)$ a broken holomorphic curve. Let $\Phi_{k}:[-k, 0] \rightarrow[a, b]$ be a sequence of diffeomorphisms satisfying hypothesis (2.1). Then conditions (c) and (d) in the definition of convergence $f_{k} \rightarrow F$ are equivalent to

$\left(\mathrm{c}^{\prime}\right) \hat{\Phi}_{k} \circ f_{k} \circ \varphi_{k}^{-1} \rightarrow \hat{\Phi} \circ \bar{F}$ in $C_{\mathrm{loc}}^{\infty}$ on $\Sigma^{*}$ (away from the nodes),

$\left(\mathrm{d}^{\prime}\right) \hat{\Phi}_{k} \circ f_{k} \circ \varphi_{k}^{-1} \rightarrow \hat{\Phi} \circ \bar{F}$ uniformly on $\Sigma$.

Proof. The equivalence of (c) and ( $\left.\mathrm{c}^{\prime}\right)$ follows immediately from $\hat{\Phi}_{k} \circ f_{k}=$ $\Phi_{k}^{(\nu)} \circ f_{k}^{(\nu)}$ and hypothesis $(2.1)$. We prove the implication $\left(\mathrm{c}^{\prime}\right),(\mathrm{d}) \Longrightarrow\left(\mathrm{d}^{\prime}\right)$. 
Let $\varepsilon>0$ be given. By hypothesis (2.1) and monotonicity of $\Phi_{k}^{(\nu)}$, there exist $R>0$ and $K_{0} \in \mathbb{N}$ such that for all $k \geq K_{0}$ and $\nu=0, \ldots, N$,

$$
\Phi_{k}^{(\nu)}(r) \geq a^{(\nu)}-\frac{\varepsilon}{2} \quad \text { for } r \geq R, \quad \Phi_{k}^{(\nu+1)}(r) \leq a^{(\nu)}+\frac{\varepsilon}{2} \quad \text { for } r \leq-R .
$$

By the definition of $\Phi_{k}^{(\nu)}$, this is equivalent to

$$
\Phi_{k}(r) \geq a^{(\nu)}-\frac{\varepsilon}{2} \quad \text { for } r \geq r_{k}^{(\nu)}+R, \quad \Phi_{k}(r) \leq a^{(\nu)}+\frac{\varepsilon}{2} \quad \text { for } r \leq r_{k}^{(\nu+1)}-R .
$$

Consider now $i \in I^{(\nu)}$. By (d), there exist $\rho_{0}>0$ and $K_{1} \geq K_{0}$ such that for all $k \geq K_{1}$ and $(s, t) \in A^{i}$ with $|s| \leq \rho_{0}$, we have $\pi_{\mathbb{R}} \circ f_{k} \circ \phi_{k}^{-1}(s, t) \in$ $\left[r_{k}^{(\nu)}+R, r_{k}^{(\nu+1)}-R\right]$, and hence

$$
\pi_{\mathbb{R}} \circ \hat{\Phi}_{k} \circ f_{k} \circ \varphi_{k}^{-1}(s, t) \in\left[a^{(\nu)}-\frac{\varepsilon}{2}, a^{(\nu)}+\frac{\varepsilon}{2}\right] .
$$

By $\pi_{\mathbb{R}} \circ \hat{\Phi} \circ \bar{F}\left(\delta^{i}\right)=a^{(\nu)}$ and continuity of $\pi_{\mathbb{R}} \circ \hat{\Phi} \circ \bar{F}$, there exists a $\rho_{1}<\rho_{0}$ such that for all $k \geq K_{1}$ and $(s, t) \in A^{i}$ with $|s| \leq \rho_{1}$, we have $\mid \pi_{\mathbb{R}} \circ \hat{\Phi} \circ$ $F(s, t)-a^{(\nu)} \mid \leq \varepsilon / 2$ and hence

$$
\left|\pi_{\mathbb{R}} \circ \hat{\Phi}_{k} \circ f_{k} \circ \varphi_{k}^{-1}(s, t)-\pi_{\mathbb{R}} \circ \hat{\Phi} \circ \bar{F}(s, t)\right| \leq \varepsilon .
$$

Together with the uniform convergence of the $M$-components in (d), this shows that for $k \geq K_{2}$ sufficiently large and every $i \in I$, the maps $\hat{\Phi}_{k} \circ f_{k} \circ$ $\varphi_{k}^{-1}$ and $\hat{\Phi} \circ \bar{F}$ are uniformly $\varepsilon$-close on some annulus $\left[-\rho_{2}, \rho_{2}\right] \times \mathbb{R} / \mathbb{Z} \subset A^{i}$. By the $C_{\text {loc }}^{\infty}$-convergence on $\Sigma^{*}$ in $\left(\mathrm{c}^{\prime}\right)$, the maps are also $\varepsilon$-close on the complement of these annuli for $k \geq K_{3}$ sufficiently large. This proves $\left(\mathrm{c}^{\prime}\right)$, $(d) \Longrightarrow\left(d^{\prime}\right)$. The converse implication is proved similarly.

Remark 2.13. Condition (d) implies properties analogous to (a) and (b) for the almost complex structures $\left(\hat{\Phi}_{k}\right)_{*} J_{k}$. Consider the almost complex structure $\hat{\Phi}_{*} J^{*}$ on $Y \backslash \Omega$, where $\Omega:=\coprod_{\nu=1}^{N}\left\{a^{(\nu)}\right\} \times M$. For the analog of (a), note that the $C_{\text {loc }}^{\infty}$-convergence $\Phi_{k}^{(\nu)} \rightarrow \Phi^{(\nu)}$ and $J_{k} \rightarrow J^{*}$ imply $C_{\text {loc }^{-}}^{\infty}$ convergence $\left(\hat{\Phi}_{k}\right)_{*} J_{k}=\left(\Phi_{k}^{(\nu)}\right)_{*} J_{k} \rightarrow \Phi_{*} J^{*}$ on $Y \backslash \Omega$. For the analog of (b), note that in view of the $C_{\mathrm{loc}}^{\infty}$-convergence $\Phi_{k}^{(\nu)} \rightarrow \Phi^{(\nu)}$, the numbers

$$
b_{k}^{(\nu)}:=\left(\Phi_{k}^{(\nu)}\right)^{-1}\left(\frac{a_{k}^{(\nu)}+a_{k}^{(\nu-1)}}{2}\right)
$$

remain bounded as $k \rightarrow \infty$. Thus $\hat{\Phi}_{k}^{-1}$ maps the cylinder

$$
\left(\left[\frac{a_{k}^{(\nu)}+a_{k}^{(\nu-1)}}{2}, \frac{a_{k}^{(\nu)}+a_{k}^{(\nu+1)}}{2}\right] \times M,\left(\Phi_{k}\right)_{*} J_{k}\right)
$$


bi-holomorphically onto the standard cylinder $\left(\left[r_{k}^{(\nu)}+b_{k}^{(\nu)}, r_{k}^{(\nu+1)}+b_{k}^{(\nu+1)}\right] \times\right.$ $\left.M, J_{M}\right)$, and the "modulus" $\left(r_{k}^{(\nu+1)}+b_{k}^{(\nu+1)}\right)-\left(r_{k}^{(\nu)}+b_{k}^{(\nu)}\right)$ tends to $\infty$ as $k \rightarrow \infty$.

Remark 2.14. If the hypersurface $M$ is disconnected, everything remains valid with the following adjustments. Denote by $M_{\iota}$ the connected components of $M$. For every $\iota$, take some number $N_{\iota}$ of copies $X_{\iota}^{(\nu)}$ of $\mathbb{R} \times M_{\iota}$ and define

$$
X^{*}:=X_{0} \amalg \coprod_{\iota} \coprod_{\nu=1}^{N_{\iota}} X_{\iota}^{(\nu)} .
$$

A broken holomorphic curve is now a collection $F=\left(F^{(0)}, F_{\iota}^{(\nu)}\right)$ of stable punctured holomorphic curves in $X_{\iota}^{(\nu)}$ which extends to a continuous map $\bar{F} \mapsto \bar{X}$, where $\bar{X}$ is obtained from $X^{*}$ as before. Now the definition of convergence and Theorem 2.9 carries over with the obvious adjustments.

Remark 2.15. If $X$ is not closed but already a symplectic cobordism, everything remains valid with the following adjustments. In the hypothesis of Theorem 2.9, the uniform area bound $\int_{\Sigma_{k}} f_{k}^{*} \omega \leq E_{0}$ must be replaced by a uniform bound on the Hofer energy (Section 4 ). Then the discussion in Sections 4 and 5 also applies to the ends $\mathbb{R}_{+} \times \bar{M}$ and $\mathbb{R}_{-} \times \underline{M}$. In this case, besides the components $F^{(\nu)}$ described in Theorem 2.9, the limit broken holomorphic curve $F$ will also have finitely many levels of punctured holomorphic curves in the symplectizations $\mathbb{R} \times \bar{M}$ and $\mathbb{R} \times \underline{M}$.

\section{Gromov compactness with free boundary}

In this section, we extend Gromov's compactness theorem to holomorphic curves with boundary which satisfy gradient bounds near the boundary. This extension is needed in the proof of the compactness Theorem 2.9. Our proof follows Gromov's original arguments and their exposition in [12]. However, the extension to the case with boundary is not entirely straightforward; it involves a detailed analysis of embedded annuli in Riemann surfaces.

Throughout this section, $(X, J, \mu)$ denotes an almost complex, not necessarily closed, manifold $(X, J)$ with a Hermitian metric $\mu$, i.e., a $J$-invariant Riemannian metric. To a map $f: \Sigma \rightarrow X$ from a compact surface, we associate its $\mu$-area $\operatorname{area}_{\mu}(f)$.

Let $\bar{\Sigma}$ be a compact surface of genus $g$ with $m$ smooth boundary components and $q$ distinct marked points $\mathbf{z}=\left(z^{1}, \ldots, z^{q}\right) \subset \operatorname{int}(\bar{\Sigma})$ in the interior of $\bar{\Sigma}$. Here $g$ is by definition the genus of the surface obtained by filling in a disk at each boundary component. Consider a finite collection $\Delta$ of disjoint simple loops in the interior of $\bar{\Sigma}$. Denote by $\Sigma$ the nodal surface obtained by collapsing the loops in $\Delta$. Thus $\Sigma$ is a finite disjoint union of smooth surfaces with finitely many pairs of points identified. As above, denote by $\Delta$ 
also the image of $\Delta$ under the projection $\pi: \bar{\Sigma} \rightarrow \Sigma$. A conformal structure $j$ on $\Sigma$ is a conformal structure on each component of $\Sigma$. The pair $(\Sigma, j)$ is called a nodal Riemann surface. A continuous map $f:(\Sigma, j) \rightarrow(X, J)$ is called a nodal holomorphic curve if its restriction to each component of $\Sigma$ is holomorphic. Moreover, we require that there is no sphere with less than three nodal or marked points on which $f$ is constant. As before, we will refer to this property as stability. Marked and nodal points will be sometimes called special points. Denote by $f: \bar{\Sigma} \rightarrow X$ its lift (which is constant on each component of $\Delta$ ).

Definition 3.1. We say that a sequence of holomorphic curves with $q$ marked points $f_{k}:\left(\Sigma_{k}, j_{k}, \mathbf{z}_{k}\right) \rightarrow(X, J)$ converges to a nodal holomorphic curve $f:(\Sigma, j, \mathbf{z}) \rightarrow(X, J)$ if there exist diffeomorphisms $\phi_{k}: \Sigma_{k} \rightarrow \bar{\Sigma}$ such that

(a) $\left(\phi_{k}\right)_{*} j_{k} \rightarrow \pi^{*} j$ in $C_{\mathrm{loc}}^{\infty}$ on $\bar{\Sigma} \backslash \Delta$ and $\phi_{k}\left(z_{k}^{l}\right) \rightarrow z^{l}$;

(b) $f_{k} \circ \phi_{k}^{-1} \rightarrow f$ in $C_{\text {loc }}^{\infty}$ on $\bar{\Sigma} \backslash(\Delta \cup \partial \Sigma)$;

(c) $f_{k} \circ \phi_{k}^{-1} \rightarrow f$ in $C_{\mathrm{loc}}^{0}$ on $\Sigma \backslash \partial \Sigma$;

(d) $\operatorname{area}_{\mu}\left(f_{k}\right) \rightarrow \operatorname{area}_{\mu}(\bar{f})$.

Here $\Delta \subset \bar{\Sigma}$ and $f: \Sigma \rightarrow X$ are as above.

We will impose the following conditions on a holomorphic curve $f:(\Sigma, j) \rightarrow(X, J)$.

(A1) $\Sigma$ is a compact Riemann surface of genus $g$ with $m$ boundary components and $q$ distinct marked points $\mathbf{z}=\left\{z^{1}, \ldots, z^{q}\right\}$ in the interior.

(A2) The $\mu$-area of $f$ is at most $C$.

(A3) The image of $f$ is contained in a compact subset $K \subset X$.

(A4) At the boundary components $\gamma$ of $(\Sigma, j)$, there exist mutually disjoint conformal embeddings $\beta^{\gamma}:[0,5 L] \times \mathbb{R} / \mathbb{Z} \hookrightarrow \Sigma \backslash \mathbf{z}$ mapping $\{0\} \times \mathbb{R} / \mathbb{Z}$ onto $\gamma$ for some $L \geq L_{0}(g, m, q, C, K) \geq 1$.

(A5) For each boundary component $\gamma$, the differential of $f \circ \beta^{\gamma}$ satisfies $1 / D \leq\left\|T\left(f \circ \beta^{\gamma}\right)\right\| \leq D$ with respect to the Euclidean metric on $[0,5 L] \times \mathbb{R} / \mathbb{Z}$ and $\mu$, for some constant $D>0$.

Here $[0,5 L] \times \mathbb{R} / \mathbb{Z}$ is equipped with the conformal structure induced from $\mathbb{C}$. Now we can state the main result of this section.

Theorem 3.2 (Gromov compactness with free boundary). Let $(X, J, \mu)$ be an almost complex manifold with Hermitian metric. Let $f_{k}:\left(\Sigma_{k}, j_{k}, \mathbf{z}_{k}\right) \rightarrow$ $(X, J)$ be a sequence of holomorphic curves with $q$ marked points satisfying ( $A 1-A 5)$ with $g, m, q, C, K, L, D$ independent of $k$. Then a subsequence of $\left(f_{k}\right)$ converges to a nodal holomorphic curve with $q$ marked points $f$ : $(\Sigma, j, \mathbf{z}) \rightarrow(X, J)$

Moreover, we can choose the maps $\phi_{k}$ such that the restricted maps $\phi_{k} \circ$ $\beta_{k}^{\gamma}:[0, L] \times \mathbb{R} / \mathbb{Z} \rightarrow \Sigma \backslash \Delta$ are independent of $k$ for all $\gamma$. 
Remark 3.3. The constant $L_{0}(g, m, q, C, K)$ in hypothesis (A4) is determined by Lemma 3.19. It depends only on $g, m, C$ in (A1 and A2) and the constants $\varepsilon_{0}, C_{\mathrm{ML}}, C_{\mathrm{SL}}$ associated below to the set $K \subset X$. These constants depend on the injectivity radius of $K$, as well as on the Monotonicity Lemma 3.17 and the Schwarz Lemma 3.18 on $K$.

3.1. Conformal modulus. The following discussion is borrowed from Ahlfors $[\mathbf{1}]$. Let $(\Sigma, j)$ be a Riemann surface, possibly with smooth boundary, and let $\Gamma$ be an isotopy class of simple smooth loops in $\Sigma$. A measurable conformal metric $\rho$ is a symmetric $(0,2)$ tensor that in local conformal coordinates is a multiple of the Euclidean metric by a non-negative measurable function. Define the minimum length of curves in $\Gamma$ by

$$
L_{\rho}(\Gamma):=\inf _{\gamma \in \Gamma} L_{\rho}(\gamma)
$$

Define the modulus of $\Gamma$ by

$$
M(\Gamma):=\inf _{\rho} \frac{\operatorname{area}_{\rho}(\Sigma)}{L_{\rho}(\Gamma)^{2}},
$$

where the infimum is taken over all measurable conformal metrics with $0<$ $L_{\rho}(\Gamma)<\infty$. Clearly, $M(\Gamma)$ is a conformal invariant. Its reciprocal, $1 / M(\Gamma)$, is known as the "extremal length."

An annulus is a surface diffeomorphic to $[0,1] \times \mathbb{R} / \mathbb{Z}$. We restrict ourselves to closed annuli, but the discussion below extends to open or semi-open annuli. By the uniformization theorem, every conformal annulus is conformally equivalent to an annulus $[0, L] \times \mathbb{R} / \mathbb{Z}$ with the standard conformal structure induced from $\mathbb{C}$.

Lemma 3.4. For an annulus $A$, let $\Gamma_{A}$ be the class of simple loops isotopic to a boundary component. If $A$ is conformally equivalent to $[0, L] \times \mathbb{R} / \mathbb{Z}$, then

$$
M\left(\Gamma_{A}\right)=L .
$$

Proof. Let $A=[0, L] \times \mathbb{R} / \mathbb{Z}$ with conformal coordinates $s+i t$. Any measurable conformal metric on $A$ can be written as $\rho=f(s, t)^{2}(d s \otimes d s+d t \otimes d t)$ for a non-negative measurable function $f: A \rightarrow \mathbb{R}$. Suppose that $L_{\rho}\left(\Gamma_{A}\right) \geq 1$. Then $\int_{0}^{1} f(s, t) d t \geq 1$ for almost all $s$ and we compute

$L \leq \int_{A} f(s, t) d s d t \leq\left(\int_{A} f(s, t)^{2} d s d t\right)^{1 / 2}\left(\int_{A} 1 d s d t\right)^{1 / 2}=\sqrt{L} \sqrt{\operatorname{area}_{\rho}(A)}$.

Thus $L \leq \operatorname{area}_{\rho}(A)$ for every measurable conformal metric $\rho$ such that $L_{\rho}\left(\Gamma_{A}\right) \geq 1$. This proves $L \leq M\left(\Gamma_{A}\right)$. Moreover, in the computation above, equality is attained if and only if $\rho$ is a constant multiple of the Euclidean metric. 
The number $M\left(\Gamma_{A}\right)$ is called the modulus of the annulus $A$ and denoted by $\operatorname{Mod}(A)$. This definition agrees with the one in $[\mathbf{1}]$ and differs from the one in $[\mathbf{1 2}]$ by a factor of $2 \pi$.

Lemma 3.5. Let $\Sigma_{0} \subset \Sigma$ be an embedded surface (with boundary) in a Riemann surface $(\Sigma, j)$ and set $\Sigma_{1}:=\Sigma \backslash \Sigma_{0}$. Let $\Gamma$ be an isotopy class of loops in $\Sigma$ and set $\Gamma_{i}:=\left\{\gamma \in \Gamma \mid \gamma \subset \Sigma_{i}\right\}$ for $i=0,1$. Then

$$
M\left(\Gamma_{0}\right)+M\left(\Gamma_{1}\right) \leq M(\Gamma)
$$

where $M\left(\Gamma_{i}\right)=0$ if $\Gamma_{i}=\emptyset$.

Proof. Let $\rho$ be a measurable conformal metric on $\Sigma$ and $\rho_{i}$ its restriction to $\Sigma_{i}$. Then clearly area $\rho_{0}\left(\Sigma_{0}\right)+$ area $_{\rho_{1}}\left(\Sigma_{1}\right) \leq$ area $_{\rho}(\Sigma)$. Moreover, since $L_{\rho_{i}}(\gamma)=L_{\rho}(\gamma)$ for every $\gamma \in \Gamma_{i}, L_{\rho_{i}}\left(\Gamma_{i}\right) \geq L_{\rho}(\Gamma)$. Hence

$$
\begin{aligned}
\frac{\operatorname{area}_{\rho}(\Sigma)}{L_{\rho}(\Gamma)^{2}} & \geq \frac{\operatorname{area}_{\rho_{0}}\left(\Sigma_{0}\right)+\operatorname{area}_{\rho_{1}}\left(\Sigma_{1}\right)}{\min \left(L_{\rho_{0}}\left(\Gamma_{0}\right)^{2}, L_{\rho_{1}}\left(\Gamma_{1}\right)^{2}\right)} \\
& \geq \frac{\operatorname{area}_{\rho_{0}}\left(\Sigma_{0}\right)}{L_{\rho_{0}}\left(\Gamma_{0}\right)^{2}}+\frac{\operatorname{area}_{\rho_{1}}\left(\Sigma_{1}\right)}{L_{\rho_{1}}\left(\Gamma_{1}\right)^{2}} \\
& \geq M\left(\Gamma_{0}\right)+M\left(\Gamma_{1}\right) .
\end{aligned}
$$

Taking the infimum on the left-hand side, the lemma follows.

In particular, if an embedded annulus $A_{0} \subset A$ in a conformal annulus $A$ is non-contractible, then $\operatorname{Mod}\left(A_{0}\right) \leq \operatorname{Mod}(A)$. Note that this is generally false if $A_{0}$ is contractible. Lemma 3.5 also immediately implies

Corollary 3.6. For any isotopy class $\Gamma$ of loops in a Riemann surface $(\Sigma, j)$, $\sup \{\operatorname{Mod}(A) \mid A \subset \Sigma$ embedded annulus containing a curve in $\Gamma\} \leq M(\Gamma)$.

Remark 3.7. In fact, the inequality in Corollary 3.6 is an equality [13]. But we will not need this deeper fact.

The following lemma and its proof were explained to us by Kerckhoff, see Figure 2.

Lemma 3.8. Let $\gamma$ be a boundary component of a Riemann surface $(\Sigma, j)$. Suppose that there exists a conformal embedding $[0, L+1 / 2] \times \mathbb{R} / \mathbb{Z} \hookrightarrow \Sigma$ mapping $\{0\} \times \mathbb{R} / \mathbb{Z}$ onto $\gamma$. Let $p \in \Sigma$ be the image of a point $(L, t)$ under this embedding. Then for every embedded annulus $A \subset \Sigma \backslash\{p\}$ whose boundary is isotopic to $\gamma$ in $\Sigma \backslash\{p\}$,

$$
\operatorname{Mod}(A) \leq L+\frac{1}{2}
$$

Remark 3.9. Note that the modulus of $A$ is surprisingly close to $L$. If in the hypothesis $L+1 / 2$ is replaced by $L+r$ with large $r$, the estimate can be further sharpened. Ahlfors [1] shows that in the limit $r \rightarrow \infty$, one can 


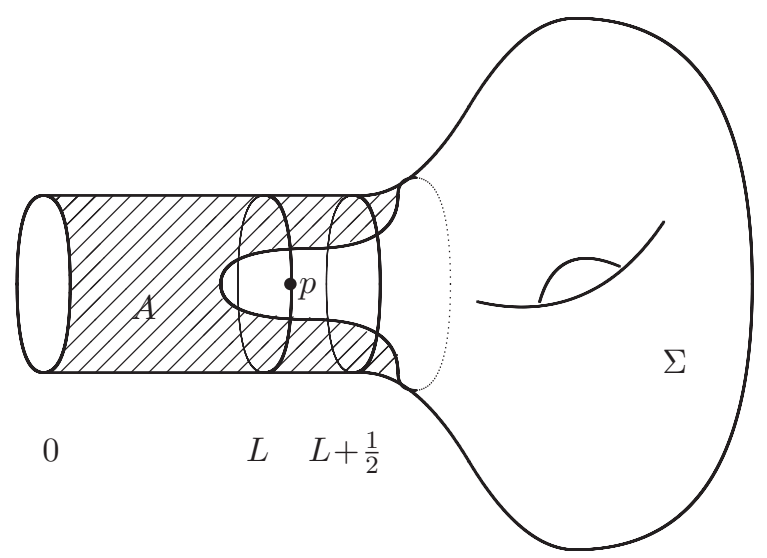

Figure 2. Embedded annulus adjacent to a boundary component.

replace $1 / 2$ in the conclusion by $\ln 16 / 4 \pi$. For our purposes, however, any universal constant in place of $1 / 2$ would suffice.

Proof. Denote the images of $[0, L] \times \mathbb{R} / \mathbb{Z}$ and $[L, L+1 / 2] \times \mathbb{R} / \mathbb{Z}$ under the embedding by $A_{0}$ and $A_{1}$, respectively. Note that $\operatorname{Mod}\left(A_{0}\right)=L$ and $\operatorname{Mod}\left(A_{1}\right)=1 / 2$. Consider the isotopy class $\Gamma$ of simple loops in $\Sigma \backslash\{p\}$ that are isotopic to $\gamma$ in $\Sigma \backslash\{p\}$. Corollary 3.6 implies

$$
\operatorname{Mod}(A) \leq M(\Gamma)=\inf _{\rho} \frac{\operatorname{area}_{\rho}(\Sigma \backslash\{p\})}{L_{\rho}(\Gamma)^{2}} .
$$

Thus we can estimate $\operatorname{Mod}(A)$ from above by picking a particular conformal metric on $\Sigma \backslash\{p\}$. Let $\rho$ be the measurable conformal metric on $\Sigma$ which corresponds to the Euclidean metric on $A_{0} \cup A_{1}$ and equals zero outside.

Let $\lambda$ be a loop in $\Gamma$ (e.g., $\lambda$ could be a boundary component of the annulus $A$ in Figure 2).

If $\lambda$ is contained in $A_{0} \cup A_{1}$, its $\rho$-length is at least 1 . Otherwise, $\lambda$ contains two arcs in $A_{1}$ connecting its two boundary components $\partial_{0} A_{1}, \partial_{1} A_{1}$ and we obtain $L_{\rho}(\lambda) \geq 2 \operatorname{dist}\left(\partial_{0} A_{1}, \partial_{1} A_{1}\right)=1$. This shows that $L_{\rho}(\Gamma) \geq 1$. On the other hand, the $\rho$-area of $\Sigma$ equals $L+1 / 2$, and therefore

$$
\operatorname{Mod}(A) \leq \frac{\operatorname{area}_{\rho}(\Sigma \backslash\{p\})}{L_{\rho}(\Gamma)^{2}}=L+\frac{1}{2}
$$

3.2. Hyperbolic surfaces. Here we follow the discussion in Chapter IV of [12]. A hyperbolic surface $(\Sigma, h)$ is a complete oriented surface with a metric of curvature -1 having geodesic boundary and finite area. Its topology is determined by its signature $(g, m, l)$. Here $m$ is the number of boundary components, $l$ is the number of ends and $g$ is the genus of the 


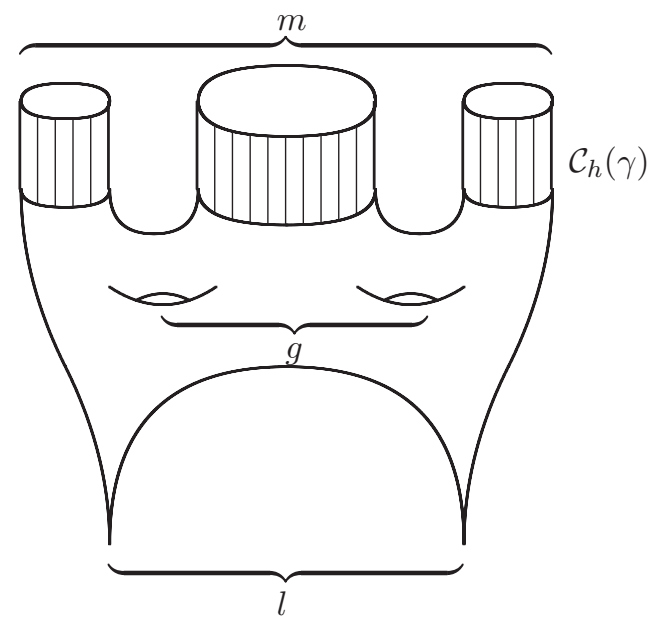

Figure 3. Riemann surface of genus $g$ with $m$ boundary components and $l$ cusps.

surface obtained by filling in a disk at each boundary geodesic and a point at each end, see Figure 3. Denote by $j$ the conformal structure induced by $h$. Since each end is part of a standard cusp ([12], Proposition IV.3.6), $j$ extends uniquely to a conformal structure on the compact surface obtained by adding a point at each end. Hence $(\Sigma, j)$ is conformally equivalent to a closed surface of genus $g$ with $m$ open disks and $l$ points removed. Its Euler characteristic is given by $\chi(\Sigma)=2-2 g-m-l<0$. Conversely, by the uniformization theorem, every closed Riemann surface of genus $g$ with $m$ open disks and $l$ points removed carries a unique compatible hyperbolic metric, provided that $2-2 g-m-l<0$.

Lemma 3.10. Let $(\Sigma, h)$ be a hyperbolic surface and let $A \subset \Sigma$ be an embedded annulus containing a simple closed (interior or boundary) geodesic $\gamma$. Then

$$
L_{h}(\gamma)^{2} \leq \frac{-2 \pi \chi(\Sigma)}{\operatorname{Mod}(A)}
$$

Proof. Since $\gamma$ minimizes the length in its isotopy class $\Gamma$ (see, e.g., Theorem 4.3.2. in [14]), Corollary 3.6 yields

$$
L_{h}(\gamma)^{2}=L_{h}(\Gamma)^{2} \leq \frac{\operatorname{area}_{h}(\Sigma)}{M(\Gamma)} \leq \frac{\operatorname{area}_{h}(\Sigma)}{\operatorname{Mod}(A)} .
$$

Now the lemma follows because by the Gauss-Bonnet theorem (see [12], Proposition IV.2.8) the area of a hyperbolic surface equals $-2 \pi \chi(\Sigma)$.

The following lemma is proved in [12], Lemma IV.1.5, Lemma IV.1.6 and Proposition IV.2.2, see Figure 3. 
Lemma 3.11. Let $(\Sigma, h)$ be a hyperbolic surface. Then its boundary geodesics $\gamma$ possess mutually disjoint embedded collars $\mathcal{C}_{h}(\gamma)=\{z \in \Sigma \mid$ $\left.d_{h}(z, \gamma)<w_{h}(\gamma)\right\}$ whose widths $w_{h}(\gamma)$ satisfy

$$
\sinh w_{h}(\gamma) \cdot \sinh L_{h}(\gamma)=1
$$

Next we recall the compactness theorem for hyperbolic surfaces. Consider a surface $\bar{\Sigma}$ of signature $(g, m, l)$ and a finite collection $\Delta$ of disjoint simple non-contractible (interior or boundary) loops of $\bar{\Sigma}$. Denote by $\Sigma$ the nodal surface obtained by collapsing the loops in $\Delta$. Thus $\Sigma$ is a finite disjoint union of smooth surfaces with finitely many pairs of points identified. As before, we denote by $\Delta$ also the image of $\Delta$ under the projection $\bar{\Sigma} \rightarrow \Sigma$. A hyperbolic structure $h$ on $\Sigma$ is a hyperbolic structure on $\Sigma \backslash \Delta$. The pair $(\Sigma, h)$ is called a nodal hyperbolic surface.

We say that a sequence $\left(\Sigma_{k}, h_{k}\right)$ of hyperbolic surfaces converges to a nodal hyperbolic surface $(\Sigma, h)$ if there exist collections $\Delta_{k} \subset \Sigma_{k}$ of simple $h_{k}$-geodesics and diffeomorphisms $\phi_{k}: \Sigma_{k} \rightarrow \bar{\Sigma}$ with $\phi_{k}\left(\Delta_{k}\right)=\Delta$ such that $\left(\phi_{k}\right)_{*} h_{k} \rightarrow h$ in $C_{\text {loc }}^{\infty}$ on $\bar{\Sigma} \backslash \Delta$ where we denote by $h$ the pull-back of $h$ under the projection $\bar{\Sigma} \rightarrow \Sigma$. For the proof of the following result, see [12], Proposition IV.5.1 (the result is formulated there only in the case where no pinching of interior geodesics occurs; the general case follows by applying this special case to all the pieces in a pair-of-pants decomposition, cf. [12], Theorem IV.3.7).

Proposition 3.12 (compactness of hyperbolic surfaces). Let $\left(\Sigma_{k}, h_{k}\right)$ be a sequence of hyperbolic surfaces of the same signature. Suppose that the lengths of all boundary geodesics are uniformly bounded from above. Then a subsequence converges to a nodal hyperbolic surface.

Remark 3.13. Consider a sequence $\left(\Sigma_{k}, j_{k}\right)$ of compact Riemann surfaces of genus $g$ with $m$ boundary components. Fix an integer $l \geq 0$ such that $2-2 g-m-l<0$. For each $k$, let $F_{k} \subset \Sigma_{k}$ be a set of $l$ non-boundary points. Let $h_{k}$ be the unique hyperbolic metric on $\Sigma_{k} \backslash F_{k}$, and suppose that the lengths of all boundary geodesics of $\left(\Sigma_{k} \backslash F_{k}, h_{k}\right)$ are uniformly bounded from above. Then Proposition IV.5.1 in $[\mathbf{1 2}]$ yields the following refined result. After passing to a subsequence, there exists a compact surface $\bar{\Sigma}$ of genus $g$ with $m$ boundary components, a subset $F \subset \bar{\Sigma}$ with $|F|=\left|F_{k}\right|$, a finite set $\Delta$ of disjoint simple non-contractible loops in $\Sigma \backslash F$ and diffeomorphisms $\phi_{k}: \Sigma_{k} \rightarrow \bar{\Sigma}$ with $\phi_{k}\left(F_{k}\right)=F$ and $\phi_{k}\left(\Delta_{k}\right)=\Delta$ for collections $\Delta_{k}$ of simple $h_{k}$-geodesics such that the following holds. Let $\Sigma$ be the nodal surface obtained by collapsing the loops in $\Delta$. Denote by $\Delta$ and $F$ also the images of $\Delta$ and $F$ in $\Sigma$. Then there exist a hyperbolic metric $h$ on $\Sigma \backslash(F \cup \Delta)$ and a compatible conformal structure $j$ on $\Sigma \backslash \Delta$ such that

- $\left(\phi_{k}\right)_{*} h_{k} \rightarrow h$ in $C_{\text {loc }}^{\infty}$ on $\bar{\Sigma} \backslash(F \cup \Delta)$ and

- $\left(\bar{\phi}_{k}\right)_{*} j_{k} \rightarrow j$ in $C_{\mathrm{loc}}^{\infty}$ on $\bar{\Sigma} \backslash \Delta$. 
Define convergence to a nodal Riemann surface as convergence of nodal holomorphic curves in $X=$ pt. In view of Lemma 3.10, the preceding remark implies

Corollary 3.14 (compactness of Riemann surfaces). Let $\left(\Sigma_{k}, j_{k}\right)$ be a sequence of compact Riemann surfaces of the same genus and with the same number of boundary components. Suppose that all boundary components are contained in mutually disjoint embedded annuli whose moduli are uniformly bounded from below. Then a subsequence converges to a nodal Riemann surface.

3.3. Thick-thin decomposition. Let $(\Sigma, h)$ be a hyperbolic surface without boundary. For $0<\delta<\operatorname{arsinh}(1)$, define the $\delta$-thin part of $(\Sigma, h)$ as the set of all points at which the injectivity radius is less than $\delta$ and the $\delta$-thick part as its complement. The following lemma is proved in [12], Proposition IV.4.2 and Example I.5.5.

Lemma 3.15. For $0<\delta<\operatorname{arsinh}(1)$, every component of the $\delta$-thin part of a hyperbolic surface without boundary is conformally equivalent to a punctured disk or an annulus. Moreover, for all $0<\delta<\operatorname{arsinh}(1)$ and $L>0$, there exists a $\delta^{\prime}>0$ (depending only on $\delta, L$ ) such that every component of the $\delta^{\prime}$-thin part is contained in an annulus $A$ of modulus $\operatorname{Mod}(A)>5 L$ which is contained in the $\delta$-thin part.

For $z \in \Sigma$, let $B_{\rho}(z):=\left\{z^{\prime} \in \Sigma \mid d_{h}\left(z, z^{\prime}\right)<\rho\right\}$. Note that $B_{\rho}(z)$ is the image of an isometric immersion of a hyperbolic disk of radius $\rho$. The following lemma is proved in [12] (replacing $\operatorname{arsinh}(1)$ by $\delta$ in the proof of Lemma V.2.3), see Figure 4.

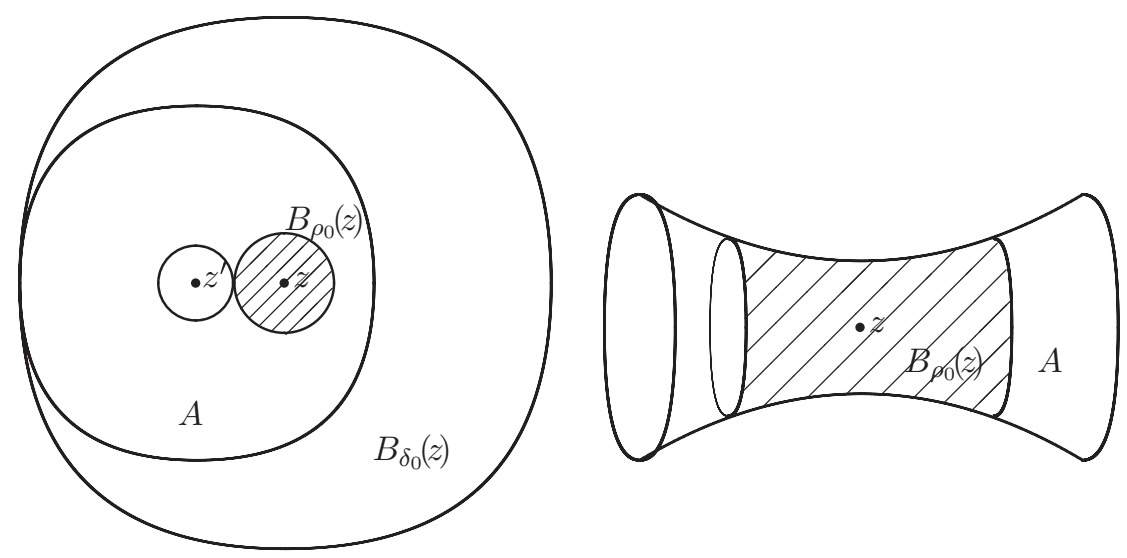

Figure 4. $\delta_{0}$-thick part (left) and $\delta_{0}$-thin part (right). 
Lemma 3.16. For any positive constants $C, L, \delta, \varepsilon$, there exists a $\rho_{0}>0$ (depending only on $C, L, \delta, \varepsilon$ ) such that $\delta_{0}:=2 \sqrt{\rho_{0}}<\delta$ and the following holds. For any hyperbolic surface without boundary $(\Sigma, h)$, any point $z \in$ $\Sigma$, and any holomorphic map $f:(\Sigma, h) \rightarrow(X, J, \mu)$ to an almost complex manifold with Hermitian metric with $\operatorname{area}_{\mu}(f) \leq C$, there exists an embedded annulus $A \subset \Sigma$ with the following properties.

(i) $B_{\rho_{0}}(z) \subset A$.

(ii) $\operatorname{Mod}(A)>3 L$.

(iii) $L_{\mu}\left(f\left(\partial_{i} A\right)\right)<\varepsilon$ for both boundary components $\partial_{0} A, \partial_{1} A$ of $A$.

(iv) If $z$ lies in the $\delta_{0}$-thick part, then $A \subset B_{\delta_{0}}(z)$ is contractible and can be chosen to enclose a given point $z^{\prime}$ with $d_{h}\left(z, z^{\prime}\right)=2 \rho_{0}$.

(v) If $z$ lies in the $\delta_{0}$-thin part, then $A$ is contained in the $\delta$-thin part and is non-contractible.

3.4. Monotonicity and Schwarz Lemma. Let $(X, J, \mu)$ be an almost complex manifold with Hermitian metric $\mu$ and $K \subset X$ a compact subset. The following two lemmas were proved by Gromov $[\mathbf{6}]$, see Chapter II in [12] for more detailed proofs.

Lemma 3.17 (Monotonicity Lemma). There exist constants $\varepsilon_{\mathrm{ML}}, C_{\mathrm{ML}}>0$ such that for any $J$-holomorphic map $f: \Sigma \rightarrow X$ from a compact Riemann surface, passing though a point $x \in K$ and with $f(\partial \Sigma)$ outside the $\mu$-ball $B_{\rho}(x)$ of radius $\rho<\varepsilon_{\mathrm{ML}}$, we have

$$
\operatorname{area}_{\mu}\left(B_{\rho}(x) \cap f(\Sigma)\right) \geq C_{\mathrm{ML}} \rho^{2} .
$$

For a map $f: D \rightarrow X$ from the unit disk, denote by $\left\|T_{0} f\right\|_{\mu}$ the norm of its differential at 0 , measured with respect to the hyperbolic metric on the disk and the metric $\mu$.

Lemma 3.18 (Schwarz Lemma). There exist constants $\varepsilon_{\mathrm{SL}}, C_{\mathrm{SL}}>0$ such that for any $J$-holomorphic map $f: D \rightarrow X$ whose image is contained in a $\mu$-ball of radius $\varepsilon_{\mathrm{SL}}$ centered at $x \in K$,

$$
\left\|T_{0} f\right\|_{\mu} \leq C_{\mathrm{SL}} \text {. }
$$

As in [12], Remark II.4.3, we pick an $\varepsilon_{0}>0$ satisfying the following conditions:

(i) $\varepsilon_{0}$ is smaller than the $\mu$-injectivity radius of $K$,

(ii) $\varepsilon_{0}$ is smaller than the constants $\varepsilon_{\mathrm{ML}}, \varepsilon_{\mathrm{SL}}$ in the Monotonicity resp. Schwarz Lemma,

(iii) every closed $J$-holomorphic curve in $X$ whose image is contained in a $\mu$-ball of radius $\varepsilon_{0}$ centered at $x \in K$ is constant.

3.5. Gradient bounds. Let $(X, J, \mu)$ be an almost complex manifold with Hermitian metric $\mu$. Let $f:(\Sigma, j) \rightarrow(X, J)$ be a holomorphic curve satisfying conditions $(\mathrm{A} 1-\mathrm{A} 5)$. Note that since $f \circ \beta^{\gamma}$ is holomorphic, condition 
(A5) is equivalent to

$$
\frac{\|v\|_{\text {Eucl }}}{D} \leq\left\|T\left(f \circ \beta^{\gamma}\right) \cdot v\right\|_{\mu} \leq D\|v\|_{\text {Eucl }}
$$

for all $v \in T([0,5 L] \times \mathbb{R} / \mathbb{Z})$.

Let $\varepsilon_{0}, C_{\mathrm{ML}}, C_{\mathrm{SL}}$ be the constants associated above to the compact set $K \subset X$ (arising in the Monotonicity and Schwarz Lemma). For $0<l \leq 5 L$, set

$$
A_{l}(\gamma):=\beta^{\gamma}\left([0, l] \times \frac{\mathbb{R}}{\mathbb{Z}}\right), \quad A_{l}:=\cup_{\gamma} A_{l}(\gamma) .
$$

Now we proceed as in Gromov's original compactness proof, following Chapter $\mathrm{V}$ of $[\mathbf{1 2}]$. For $z \in \Sigma$, denote by $U(z)$ the connected component of $f^{-1} B_{\varepsilon_{0} / 18}(f(z))$ containing $z$. Pick a maximal set $F$ of points in $\Sigma \backslash\left(\right.$ int $\left.A_{L} \cup \mathbf{z}\right)$ such that for any $z \in F, U(z)$ is disjoint from $U\left(z^{\prime}\right)$ and $U\left(z^{i}\right)$ for any $z^{\prime} \in F \backslash\{z\}$ and any marked point $z^{i} \in \mathbf{z}$. By the Monotonicity Lemma 3.17, the number of points is bounded by $\left(18 / \varepsilon_{0}\right)^{2} C / C_{\mathrm{ML}}$, where $C$ is the constant from (A2). Now enlarge $F$ so that it contains points of $\partial A_{L}(\gamma), \partial A_{2 L}(\gamma)$ and $\partial A_{4 L}(\gamma)$ for each boundary geodesic $\gamma$ and all marked points. Moreover, pick enough points such that the punctured surface $\dot{\Sigma}:=\Sigma \backslash F$ has negative Euler characteristic. The cardinality $|F|$ is still bounded by a constant depending only on $m, q, C, \varepsilon_{0}$ and $C_{\mathrm{ML}}$. Notice that the Riemann surface $\dot{\Sigma}$ depends on $L$. Let $h$ be the unique hyperbolic metric on $\dot{\Sigma}$ compatible with the conformal structure.

Lemma 3.19. There exist constants $L_{0} \geq 1$ and $l_{+}, w_{-}>0$ depending only on $g, m, q, C, \varepsilon_{0}$ and $C_{\mathrm{ML}}$, and for every $L \geq L_{0}$, there exist constants $l_{-}, w_{+}>0$ depending only on $L$, such that the following holds. For every $J$-holomorphic curve $f$ satisfying $(A 1-A 5)$ and $(\dot{\Sigma}, h)$ as above, there exist mutually disjoint collars $\mathcal{C}_{h}(\gamma) \subset \dot{\Sigma}$ around the boundary geodesics of $(\dot{\Sigma}, h)$ of width $w_{h}(\gamma)$ such that for all boundary geodesics $\gamma$,

$$
l_{-} \leq L_{h}(\gamma) \leq l_{+}, \quad w_{-} \leq w_{h}(\gamma) \leq w_{+} .
$$

Moreover, we may assume $\mathcal{C}_{h}(\gamma) \subset A_{L}(\gamma)$.

Proof. By hypothesis, each boundary geodesic $\gamma$ of $(\dot{\Sigma}, h)$ is contained in an annulus of modulus $L_{0}$. Hence by Lemma 3.10, its length is bounded from above by $l_{+}:=\sqrt{-2 \pi \chi(\dot{\Sigma}) / L_{0}}$. By this bound and Lemma 3.11, the boundary geodesics possess mutually disjoint collars $\mathcal{C}_{h}(\gamma)$ whose widths are bounded from below by a constant $w_{-}$depending only on $l_{+}$. Note that $l_{+} \rightarrow 0$ and $w_{-} \rightarrow \infty$ as $L_{0} \rightarrow \infty$. Moreover, the width and the modulus of a collar are related by an increasing bijective function $\mathbb{R}_{+} \rightarrow \mathbb{R}_{+}$(see [12], Proposition IV.2.2 and Example I.5.5). So if $L_{0}$ is sufficiently large, then each collar will have modulus at least 1 . 
Consider a collar $\mathcal{C}=\mathcal{C}_{h}(\gamma)$. Let $\mathcal{C}_{0} \subset \mathcal{C}$ be a smaller collar such that $\operatorname{Mod}\left(\mathcal{C}_{0}\right)<L_{0}-1 / 2$ and $\operatorname{Mod}\left(\mathcal{C} \backslash \mathcal{C}_{0}\right) \geq 1 / 2$. Suppose that $\mathcal{C}_{0}$ is not contained in $A_{L_{0}}(\gamma)$. Then there exists a point $p \in \partial \mathcal{C}_{0}$ which is not contained in $A_{L_{0}}(\gamma)$. Applying Lemma 3.8 with $A=A_{L_{0}}(\gamma)$ and $L=\operatorname{Mod}\left(\mathcal{C}_{0}\right)$, we conclude $L_{0}=\operatorname{Mod}\left(A_{L_{0}}(\gamma)\right) \leq \operatorname{Mod}\left(\mathcal{C}_{0}\right)+1 / 2$, contradicting the choice of $\mathcal{C}_{0}$. Hence $\mathcal{C}_{0} \subset A_{L_{0}}(\gamma)$, so after replacing the $\mathcal{C}_{h}(\gamma)$ by smaller collars, we may assume $\mathcal{C}_{h}(\gamma) \subset A_{L_{0}}(\gamma)$.

Now let $L \geq L_{0}$. Remember that in $\dot{\Sigma}$ we have removed a point of $\partial A_{L}(\gamma)$ for each boundary geodesic $\gamma$. Hence by Lemma 3.8, applied to the nonpunctured surface $(\Sigma, j)$, every embedded annulus $A$ in $\dot{\Sigma}$ with one boundary component $\gamma$ has modulus $\operatorname{Mod}(A) \leq L+1 / 2$. Applying this to the collar $\mathcal{C}_{h}(\gamma) \subset \dot{\Sigma}$ at $\gamma$, we see that its modulus is at most $L+1 / 2$. Since the width and the modulus of a collar are related by an increasing bijective function $\mathbb{R}_{+} \rightarrow \mathbb{R}_{+}$, the width is bounded from above by a constant $w_{+}$depending only on $L$. Now again by Lemma 3.11, the length of each boundary geodesic is bounded from below by a universal constant $l_{-}$depending only on $L$.

Lemma 3.20. Let $A \subset \dot{\Sigma}$ be an annulus such that neither of its boundary components $\partial_{0} A, \partial_{1} A$ is entirely contained in $A_{2 L}$ and such that $L_{\mu}\left(f\left(\partial_{i} A\right)\right)<\varepsilon:=\min \left(\varepsilon_{0} / 6, L / D\right)$. Then $A \subset \dot{\Sigma} \backslash A_{L}$ and $f(A)$ has $\mu$-diameter smaller than $\varepsilon_{0}$.

Proof. First suppose that some boundary component $\partial_{i} A$ meets $A_{L}$. Since $\partial_{i} A$ is not contained in $A_{2 L}$, there exists a boundary geodesic $\gamma$ such that $\partial_{i} A$ meets $A_{L}(\gamma)$ and is not contained in $A_{2 L}(\gamma)$. Let $\lambda$ be an $\operatorname{arc}$ of $\left(\beta^{\gamma}\right)^{-1}\left(\partial_{i} A\right)$ that connects the two boundary components in $[L, 2 L] \times \mathbb{R} / \mathbb{Z}$. Then by condition (A5),

$$
L_{\mu}\left(f\left(\partial_{i} A\right)\right) \geq \int\left\|T\left(f \circ \beta^{\gamma}\right) \cdot \frac{d \lambda}{d t}\right\| d t \geq \frac{1}{D} L_{\mathrm{Eucl}}(\lambda) \geq \frac{L}{D},
$$

contradicting the hypothesis $L_{\mu}\left(f\left(\partial_{i} A\right)\right)<L / D$. This shows that both boundary components of $A$, and therefore $A$ itself, are contained in $\dot{\Sigma} \backslash A_{L}$.

The remaining argument carries over without changes from Chapter $\mathrm{V}$ of [12]. We first show that $d(f(z), f(\partial A))<\varepsilon_{0} / 6$ for every $z \in A$. Indeed, if $U(z)$ intersects $\partial A$, then $d(f(z), f(\partial A))<\varepsilon_{0} / 18$. Otherwise, by maximality of $F$, there exists a point $z_{0} \in F$ with $U\left(z_{0}\right) \cap U(z) \neq \emptyset$. Since $z_{0} \notin A$, there exists a point $z^{\prime} \in U\left(z_{0}\right) \cap \partial A$ and we conclude $d(f(z), f(\partial A)) \leq$ $d\left(f(z), f\left(z_{0}\right)\right)+d\left(f\left(z_{0}\right), f\left(z^{\prime}\right)\right)<\varepsilon_{0} / 6$. Since $A$ is connected,

$$
\left\{z \in A \mid d\left(f(z), f\left(\partial_{0} A\right)\right)<\frac{\varepsilon_{0}}{6}\right\} \cap\left\{z \in A \mid d\left(f(z), f\left(\partial_{1} A\right)\right)<\frac{\varepsilon_{0}}{6}\right\} \neq \emptyset,
$$

and therefore $d\left(f\left(\partial_{0} A\right), f\left(\partial_{1} A\right)\right)<\varepsilon_{0} / 3$. Combining the estimates, we find $d\left(f(z), f\left(z^{\prime}\right)\right)<\varepsilon_{0}$ for all $z, z^{\prime} \in A$. 
Let $\dot{\Sigma} \cup_{\partial \Sigma} \dot{\Sigma}$ be the hyperbolic surface without boundary obtained by gluing two copies of $\dot{\Sigma}$ along their boundary. For $0<\delta<\operatorname{arsinh}(1)$, define the $\delta$-thin part of $(\dot{\Sigma}, h)$ as the set of all points at which the injectivity radius of $\dot{\Sigma} \cup_{\partial \Sigma} \dot{\Sigma}$ is less than $\delta$ and the $\delta$-thick part as its complement. Following Chapter $\mathrm{V}$ of $[\mathbf{1 2}]$, we shall call a constant universal if it depends only on $\varepsilon_{0}, C_{\mathrm{ML}}, C_{\mathrm{SL}}, g, m, q, C, L$ and $D$.

Lemma 3.21. There exists a universal constant $\rho_{0}>0$ with the following property. Every point $z \in \dot{\Sigma} \backslash A_{2 L}$ is the center of an immersed h-disk $B \subset \dot{\Sigma} \backslash A_{L}$ of radius $\rho_{0}$ such that $f(B)$ has $\mu$-diameter $<\varepsilon_{0}$.

Proof. Pick $0<\delta<\min \left(w_{-}, l_{-} / 2\right)$. In view of Lemma 3.19, the condition $\delta<l_{-} / 2$ ensures that $\partial \Sigma$ belongs to the $\delta$-thick part. Let $\varepsilon>0$ be the constant defined in Lemma 3.20, and let $C, L$ be the constants in hypotheses $(\mathrm{A} 1-\mathrm{A} 5)$. Let $\rho_{0}>0$ and $\delta_{0}:=2 \sqrt{\rho_{0}}$ be the constants provided by Lemma 3.16.

Consider first a point $z \in \dot{\Sigma} \backslash A_{2 L}$ in the $\delta_{0}$-thick part of $\dot{\Sigma}$. By Lemma 3.19, $z \notin A_{L}$ implies $z \notin \cup_{\gamma} \mathcal{C}_{h}(\gamma)$. From this and $\delta_{0}<w_{-}$, we conclude $B_{\delta_{0}}(z) \subset$ $\dot{\Sigma} \backslash \partial \Sigma$. Pick a point $z^{\prime} \in \dot{\Sigma} \backslash A_{2 L}$ with $d_{h}\left(z, z^{\prime}\right)=2 \rho_{0}$. Let $A \subset B_{\delta_{0}}(z) \subset$ $\dot{\Sigma} \backslash \partial \Sigma$ be the annulus provided by Lemma 3.16, enclosing the point $z^{\prime}$. It contains the hyperbolic disk $B=B_{\rho_{0}}(z)$ and satisfies $L_{\mu}\left(f\left(\partial_{i} A\right)\right)<\varepsilon$. If one boundary component of $A$ was contained in $A_{2 L}$, we would have $z^{\prime} \in A_{2 L}$, contradicting the choice of $z^{\prime}$. So $A$ satisfies the hypotheses of Lemma 3.20 and we conclude that $f(A)$, and therefore also $f(B)$, has diameter $<\varepsilon_{0}$.

Next consider $z \in \dot{\Sigma} \backslash A_{2 L}$ in the $\delta_{0}$-thin part. Let $A \subset \dot{\Sigma} \cup_{\partial \Sigma} \dot{\Sigma}$ be the annulus provided by Lemma 3.16. It is contained in the $\delta$-thin part. Since $\partial \Sigma$ belongs to the $\delta$-thick part, this ensures $A \subset \dot{\Sigma} \backslash \partial \Sigma$. Moreover, $A$ is non-contractible in $\dot{\Sigma}$, it contains the immersed hyperbolic disk $B=B_{\rho_{0}}(z)$, and it satisfies $\operatorname{Mod}(A)>3 L$ and $L_{\mu}\left(f\left(\partial_{i} A\right)\right)<\varepsilon$. Now there are two cases. Case 1. $A$ is non-contractible in $\Sigma$. Then if one boundary component $\partial_{i} A$ is contained in $A_{2 L}$, it is isotopic to some boundary geodesic $\gamma$ in $A_{2 L}(\gamma)$. Now remember that the set $F$ contains a point $p \in \partial A_{2 L}(\gamma)$, thus $A \subset \Sigma \backslash\{p\}$ with boundary isotopic to $\gamma$. But then Lemma 3.8 yields $\operatorname{Mod}(A) \leq 2 L+1 / 2$, contradicting $\operatorname{Mod}(A)>3 L$. So $A$ satisfies the hypotheses of Lemma 3.20 and we conclude that $f(A)$, and therefore also $f(B)$, has diameter $<\varepsilon_{0}$.

Case 2. $A$ is contractible in $\Sigma$. Denote by $\partial_{1} A$ the outer boundary component. Since $z \notin A_{2 L}, \partial_{1} A$ cannot be contained in $A_{2 L}$. A length estimate as in the proof of Lemma 3.20 shows that $\partial_{1} A \subset \dot{\Sigma} \backslash A_{L}$. This implies $A \subset \dot{\Sigma} \backslash A_{L}$. Now the last part of the proof of Lemma 3.20 shows that $f(A)$, and therefore also $f(B)$, has diameter $<\varepsilon_{0}$.

By the Schwarz Lemma 3.18 and rescaling, the differential of $f$ at the center of $B$ in the preceding lemma is universally bounded with respect to the metrics $h$ and $\mu$. This proves 
Proposition 3.22. Let $f:(\Sigma, j) \rightarrow(X, J, \mu)$ satisfy $(A 1-A 5)$. Then there exists a finite set $F \subset \Sigma \backslash A_{L}$ such that the differential of $f$ on $\Sigma \backslash\left(F \cup A_{2 L}\right)$ is universally bounded with respect to the hyperbolic metric $h$ and the Hermitian metric $\mu$. Moreover, the number of points in $F$ is universally bounded.

Corollary 3.23. There exists a universal constant $\delta_{1}>0$ such that no simple loop $\lambda$ in $\dot{\Sigma}$ with $L_{h}(\lambda) \leq 2 \delta_{1}$ which is non-contractible in $\Sigma$ meets $A_{3 L}$.

Proof. Let $\delta$ be the constant defined in the proof of Lemma 3.21. Pick $\delta_{1}<\delta^{\prime}$, where $\delta^{\prime}$ is the constant provided by Lemma 3.15. Let $\lambda$ be a simple loop in $\dot{\Sigma}$ with $L_{h}(\lambda) \leq 2 \delta_{1}$ which is non-contractible in $\Sigma$. Suppose first that $\lambda$ is contained in $A_{4 L}$. Since $\lambda$ is non-contractible in $\Sigma$, it must be isotopic to some boundary geodesic $\gamma$ in $A_{4 L}(\gamma)$. Hence by Lemma 3.8, $\operatorname{Mod}(A) \leq 4 L+1 / 2$ for every annulus in $\dot{\Sigma}$ containing $\lambda$. On the other hand, $\lambda$ is contained in the $\delta_{1}$-thin part and $\delta_{1}<\delta^{\prime}$. Hence, by Lemma 3.15, $\lambda$ is contained in an annulus $A \subset \dot{\Sigma} \cup_{\partial \Sigma} \dot{\Sigma}$ of $\operatorname{modulus} \operatorname{Mod}(A) \geq 5 L$ which is contained in the $\delta$-thin part. Since (by the choice of $\delta$ ) $\partial \Sigma$ belongs to the $\delta$-thick part, it follows that $A \subset \dot{\Sigma} \backslash \partial \Sigma$, and we get the contradiction $\operatorname{Mod}(A) \leq 4 L+1 / 2$.

Now suppose that $\lambda$ is not contained in $A_{4 L}(\gamma)$ and meets $A_{3 L}(\gamma)$. Let $\lambda_{1}$ be an arc of $\left(\beta^{\gamma}\right)^{-1} \circ \lambda$ that connects the two boundary components in $[3 L, 4 L] \times \mathbb{R} / \mathbb{Z}$. Then

$$
L_{\mu}\left(f \circ \beta^{\gamma}\left(\lambda_{1}\right)\right)=\int\left\|T\left(f \circ \beta^{\gamma}\right) \cdot \frac{d \lambda_{1}}{d t}\right\| d t \geq \frac{1}{D} L_{\text {Eucl }}\left(\lambda_{1}\right) \geq \frac{L}{D} .
$$

On the other hand, by Proposition 3.22, the differential of $f$ with respect to the hyperbolic metric satisfies $\|T f\| \leq U$ on $\dot{\Sigma} \backslash A_{2 L}$, for some universal constant $U$. Thus

$$
L_{\mu}\left(f \circ \beta^{\gamma}\left(\lambda_{1}\right)\right) \leq U L_{h}(\lambda) \leq 2 U \delta_{1} .
$$

For $\delta_{1}$ small, these two inequalities contradict each other.

Proof of Theorem 3.2. Let $(X, J, \mu)$ and $f_{k}:\left(\Sigma_{k}, j_{k}\right) \rightarrow(X, J)$ be as in Theorem 3.2. For $0<l \leq 5 L$, let $Z_{l}$ be the disjoint union of $m$ copies of $[0, l] \times \mathbb{R} / \mathbb{Z}$. Denote by $\beta_{k}: Z_{5 L} \rightarrow \Sigma_{k}$ the embedding induced by the $\beta_{k}^{\gamma}$.

For each $k$, pick a finite subset $F_{k} \subset \Sigma_{k} \backslash \beta_{k}\left(Z_{L}\right)$ as in Proposition 3.22 and a constant $\delta_{1}$ as in Corollary 3.23. After passing to a subsequence, we may assume that the cardinality $\left|F_{k}\right|$ is the same for all $k$. By Lemma 3.19, the lengths of the boundary geodesics for the hyperbolic metrics $h_{k}$ on $\Sigma_{k} \backslash F_{k}$ are uniformly bounded. Hence, by Proposition 3.12, a subsequence of $\left(\Sigma_{k} \backslash\right.$ $\left.F_{k}, h_{k}\right)$ converges to a nodal hyperbolic surface $(\bar{\Sigma}, \bar{h})$. More precisely, by the remark following Proposition 3.12, the following holds. After passing to a subsequence, there exists a compact surface $\bar{\Sigma}$ of genus $g$ with $m$ boundary components, a subset $F \subset \bar{\Sigma}$ with $|F|=\left|F_{k}\right|$, a finite set $\Delta$ of disjoint simple non-contractible loops in $\bar{\Sigma} \backslash F$ and diffeomorphisms $\phi_{k}: \Sigma_{k} \rightarrow \bar{\Sigma}$ 
with $\phi_{k}\left(F_{k}\right)=F$ and $\phi_{k}\left(\Delta_{k}\right)=\Delta$ for collections $\Delta_{k}$ of simple $h_{k}$-geodesics such that the following holds. Let $\Sigma$ be the nodal surface obtained by collapsing the loops in $\Delta$. As above, denote by $\Delta$ and $F$ also the images in $\Sigma$. Then there exist a hyperbolic metric $h$ on $\Sigma \backslash(F \cup \Delta)$ and a compatible conformal structure $j$ on $\Sigma \backslash \Delta$ such that

- $\left(\phi_{k}\right)_{*} h_{k} \rightarrow h$ in $C_{\text {loc }}^{\infty}$ on $\bar{\Sigma} \backslash(F \cup \Delta)$ and

- $\left(\phi_{k}\right)_{*} j_{k} \rightarrow j$ in $C_{\mathrm{loc}}^{\infty}$ on $\bar{\Sigma} \backslash \Delta$,

with the same conventions for $h$ and $j$ as above. Define

$$
\bar{h}_{k}:=\left(\phi_{k}\right)_{*} h_{k}, \quad \bar{\jmath}_{k}:=\left(\phi_{k}\right)_{*} j_{k},
$$

and consider the holomorphic maps

$$
\begin{aligned}
& \bar{f}_{k}:=f_{k} \circ \phi_{k}^{-1}:\left(\bar{\Sigma} \backslash \Delta, \bar{\jmath}_{k}\right) \rightarrow(X, J), \\
& \bar{\beta}_{k}:=\phi_{k} \circ \beta_{k}:\left(Z_{5 L}, i\right) \rightarrow\left(\bar{\Sigma} \backslash \Delta, \bar{\jmath}_{k}\right) .
\end{aligned}
$$

By Proposition 3.22, the differential of $\bar{f}_{k}$ is uniformly bounded with respect to $\bar{h}_{k}$ and $\mu$ on $\bar{\Sigma}_{0}:=\bar{\Sigma} \backslash\left(F \cup \Delta \cup \bar{\beta}_{k}\left(Z_{2 L}\right)\right)$. By the Arzela-Ascoli theorem and standard elliptic estimates, it follows that a subsequence of $\bar{f}_{k}$ converges in $C_{\text {loc }}^{\infty}$ on $\bar{\Sigma}_{0}$ to a holomorphic map $f:\left(\bar{\Sigma}_{0}, j\right) \rightarrow(X, J)$. By the uniform area bound and removal of singularities (see [12], Theorem III.2.1), $f$ extends smoothly over the points of $F$ and $\bar{f}_{k} \rightarrow f$ converges in $C_{\mathrm{loc}}^{\infty}$ on $\bar{\Sigma} \backslash(\Delta \cup$ $\left.\bar{\beta}_{k}\left(Z_{2 L}\right)\right)$.

By Corollary 3.23, $\bar{\beta}_{k}\left(Z_{4 L}\right)$ does not meet any component of the $\delta_{1}$-thin part of $\left(\Sigma, h_{k}\right)$ containing a geodesic from $\Delta_{k}$. Thus the $\bar{\beta}_{k}\left(Z_{4 L}\right)$ are contained in a uniform compact subset of $\bar{\Sigma} \backslash \Delta$. Hence a subsequence of $\bar{\beta}_{k}$ converges in $C^{\infty}$ on $Z_{3 L}$ to a holomorphic map $\beta:\left(Z_{3 L}, i\right) \rightarrow(\bar{\Sigma} \backslash \Delta, j)$. (This is a special case of Gromov compactness with Lagrangian boundary conditions with the Lagrangians being curves in surfaces). Since the $\bar{\beta}_{k}$ are embeddings, so is $\beta$ (otherwise $\beta$ would be a branched covering, contradicting injectivity of $\bar{\beta}_{k}$ for large $k$ ).

By hypothesis (A5) and the Arzela-Ascoli theorem, a subsequence of $\bar{f}_{k} \circ \bar{\beta}_{k}$ converges in $C_{\mathrm{loc}}^{\infty}$ on the interior of $Z_{3 L}$. Since $\bar{\beta}_{k}$ converges to an embedding $\beta$, this implies $C_{\mathrm{loc}}^{\infty}$-convergence of $\bar{f}_{k}$ on the interior of $\bar{\beta}\left(Z_{3 L}\right)$ to a map $f$ which agrees with the previous map on $\bar{\beta}\left(Z_{3 L} \backslash Z_{2 L}\right)$. Altogether, we have shown $C_{\text {loc }}^{\infty}$-convergence $\bar{f}_{k} \rightarrow f$ on $\bar{\Sigma} \backslash \Delta$ to a $(j, J)$-holomorphic map $f$. This proves properties (a) and (b) of the convergence to the nodal holomorphic curve $f$. Now properties (c) and (d) follow as in Chapter V of [12].

It remains to show that we can make the maps $\phi_{k} \circ \beta_{k}: Z_{L} \rightarrow \bar{\Sigma} \backslash \Delta$ independent of $k$. Recall that the embeddings $\bar{\beta}_{k}$ converge in $C^{\infty}$ on $Z_{3 L}$ to an embedding $\beta: Z_{3 L} \rightarrow \bar{\Sigma} \backslash \Delta$. So we can modify the $\beta_{k}$ to embeddings $\hat{\beta}_{k}: Z_{5 L} \rightarrow \bar{\Sigma}$ satisfying $\hat{\beta}_{k}=\bar{\beta}_{k}$ on $Z_{5 L} \backslash Z_{2 L}, \hat{\beta}_{k}=\beta$ on $Z_{L}$ and $\hat{\beta}_{k} \rightarrow \beta$ in 
$C^{\infty}$ on $Z_{3 L}$. Define maps $\hat{\phi}_{k}: \Sigma_{k} \rightarrow \Sigma$ by

$$
\hat{\phi}_{k}:= \begin{cases}\bar{\phi}_{k} & \text { on } \Sigma_{k} \backslash \beta_{k}\left(Z_{2 L}\right), \\ \hat{\beta}_{k} \circ \beta_{k}^{-1} & \text { on } \beta_{k}\left(Z_{5 L}\right) .\end{cases}
$$

Note that the two definitions agree on $\beta_{k}\left(Z_{5 L} \backslash Z_{2 L}\right)$, so the $\hat{\phi}_{k}$ lift to diffeomorphisms $\Sigma_{k} \rightarrow \bar{\Sigma}$. Moreover, on $Z_{L}$, the maps $\hat{\phi}_{k} \circ \beta_{k}=\beta$ are independent of $k$. The conformal structures $\hat{\jmath}_{k}:=\left(\hat{\phi}_{k}\right)_{*} j_{k}$ agree with $\bar{\jmath}_{k}$ on $\bar{\Sigma} \backslash \bar{\beta}\left(Z_{2 L}\right)$ and with $\left(\hat{\beta}_{k}\right)_{*} i$ on $\bar{\beta}_{k}\left(Z_{5 L}\right)$. In particular, $\hat{\jmath}_{k} \rightarrow \bar{\jmath}$ in $C_{\text {loc }}^{\infty}$ on $\bar{\Sigma} \backslash \Delta$. Now replace $\bar{\phi}_{k}$ by $\hat{\phi}_{k}$ and $\bar{\jmath}_{k}$ by $\hat{\jmath}_{k}$ and conclude the proof as before.

There is one more subtlety to consider. It could happen that the limit curve $f$ obtained by forgetting the additional marked points in $F$ is unstable. By construction, even the domain $\Sigma$ of the limit is stable if we include all points of $F$ into the set of marked points. Forgetting all but the $q$ marked points in $F$, a sphere on which $f$ is constant may end up with less than three special points. By the choice of $F$, such a sphere contains at most one point of $F$. Hence the image of the constant sphere can only be a double point in the image. To solve this problem, we remove these unstable components from the limiting curve while the two corresponding nodal points on the adjacent components fit into one pair of nodal points of the new curve which we will also denote by $f:(\Sigma, j, \mathbf{z}) \rightarrow(X, J)$. Notice that the corresponding surface $\bar{\Sigma}$ is diffeomorphic to the old one. However, the set $\Delta \subset \bar{\Sigma}$ of nodal curves has changed. An unstable sphere corresponds to a pair of nodal curves which bounded an annulus $A \subset \bar{\Sigma}$ parameterized by $S^{1} \times[-1,1]$, say. Let the neighborhood of $A$ be parameterized by $[-1-\varepsilon, 1+\varepsilon] \times S^{1}$ extending that parameterization and the neighborhood of the new single nodal curve $\gamma$ be parameterized by $[-\varepsilon,+\varepsilon] \times S^{1}$. Pick a sequence of diffeomorphisms $\psi_{k}:[-1-\varepsilon, 1+\varepsilon] \times S^{1} \rightarrow[-\varepsilon,+\varepsilon] \times S^{1}$, which are given by diffeomorphisms of the intervals, which are simply translations by \pm 1 near the boundaries, and which converge uniformly to

$$
\psi(s)= \begin{cases}s+1 & s \in[-1-\varepsilon,-1] \\ 0 & s \in[-1,1] \\ s-1 & s \in[1,1+\varepsilon]\end{cases}
$$

Then $\psi_{k} \circ \phi_{k}, f_{k}$ and the new stable $f: \Sigma \rightarrow(X, J)$ with its model $\bar{\Sigma}$ will satisfy Definition 3.1. Since $\psi_{k}$ converges in $C^{\infty}$ to a diffeomorphism outside $A$, it is clear that $\left(\psi_{k}^{-1}\right)_{*} \circ\left(\phi_{k}^{-1}\right)_{*} j_{k}$ and $f_{k} \circ \phi_{k}^{-1} \circ \psi_{k}^{-1}$ converge in $C_{\text {loc }}^{\infty}$ in the complement of $\gamma$ in a neighborhood of it and therefore in the complement of the new set $\Delta$. The issue here is uniform convergence across $\Delta$ which is satisfied since the sequence $f_{k} \circ \phi_{k} \mid A$ uniformly converges to the nodal point corresponding to the unstable sphere. This finishes the proof of Theorem 3.2. 


\section{Holomorphic cylinders of small area}

In this section, we establish uniform convergence of holomorphic cylinders of small area (Theorem 4.18). The main technical ingredient for this result, Proposition 4.5, is proved in [9] in the Morse case and in [3] in the MorseBott case. Moreover, we estimate the conformal modulus of a holomorphic cylinder from below (Lemma 4.20).

Throughout, $(M, \omega, J)$ is a compact odd-dimensional manifold with a stable Hamiltonian structure $\omega$ and $J$ is an $\omega$-tamed, translation invariant almost complex structure on $\mathbb{R} \times M$. Let $\lambda=\lambda_{J}$ be the associated 1-form. We assume that the closed Reeb orbits are Morse-Bott non-degenerate as defined in Section 2. Throughout this section, we fix an $\varepsilon>0$ such that

$$
(r d \lambda+\omega)(v, J v)>0 \quad \text { for } 0 \neq v \in \operatorname{ker} \lambda \text { and }|r| \leq \varepsilon .
$$

4.1. Various notions of energy. For a collection of (not necessarily embedded) smooth curves $\Gamma$ in $M$, let us denote by $A_{\lambda}(\Gamma)$ its $\lambda$-action:

$$
A_{\lambda}(\Gamma):=\int_{\Gamma} \lambda
$$

To a smooth map $f: \Sigma \rightarrow \mathbb{R} \times M$ from a (not necessarily closed) surface $\Sigma$, we associate the following quantities:

its $\omega$-energy (or area)

$$
E_{\omega}(f):=\int_{\Sigma} f^{*} \omega
$$

its $\lambda$-energy and its $(\varepsilon, \lambda)$-energy

$$
E_{\lambda}(f):=\sup _{\phi \in \mathcal{S}} \int_{\Sigma} f^{*}\left(\phi^{\prime}(r) d r \wedge \lambda\right), \quad E_{\lambda}^{\varepsilon}(f):=\sup _{\phi \in \mathcal{S}_{\varepsilon}} \int_{\Sigma} f^{*} d(\phi(r) \lambda),
$$

where $\mathcal{S}$ resp. $\mathcal{S}_{\varepsilon}$ denotes the set of all smooth non-decreasing functions $\phi: \mathbb{R} \rightarrow[0,1]$ resp. $\phi: \mathbb{R} \rightarrow[-\varepsilon, \varepsilon] ;$ its Hofer energy and $\varepsilon$-energy

$$
E(f):=E_{\omega}(f)+E_{\lambda}(f), \quad E^{\varepsilon}(f):=E_{\omega}(f)+E_{\lambda}^{\varepsilon}(f) .
$$

Note that $E_{\lambda}(f)$ and $E_{\lambda}^{\varepsilon}(f)$ are nonnegative by definition (since $\phi \equiv 0$ is allowed) and $E_{\omega}(f) \geq 0$ if $f$ is $J$-holomorphic. Here $J$-holomorphicity of a map

$$
f=(a, u):(\Sigma, j) \rightarrow \mathbb{R} \times M
$$

translates into the equations

$$
u^{*} \lambda \circ j=d a, \quad \pi T u \circ j=J_{M} \circ \pi T u,
$$

or in local holomorphic coordinates $s+i t$ on $\Sigma$,

$$
a_{s}=\lambda\left(u_{t}\right), \quad a_{t}=-\lambda\left(u_{s}\right), \quad \pi u_{s}+J \pi u_{t}=0 .
$$

Here $a_{s}$ etc. denotes partial derivatives and $\pi: T M \rightarrow \xi$ is the projection along the Reeb vector field. Denote by $\mathcal{R}$ the set of regular values of the 
map $a: \Sigma \rightarrow \mathbb{R}$. We always orient a non-empty regular level set $a^{-1}(R)$ as the boundary of the region $\{a \leq R\}$.

The following lemma shows that for holomorphic maps, the energies $E(f)$ and $E^{\varepsilon}(f)$ are equivalent.

Lemma 4.1. For a J-holomorphic map $f=(a, u):(\Sigma, j) \rightarrow \mathbb{R} \times M$, the following holds.

(a) $\int_{a^{-1}(R)} u^{*} \lambda>0$ for every $R \in \mathcal{R}$ with $a^{-1}(R) \neq \emptyset$, and

$$
E_{\lambda}(f)=\sup _{R \in \mathcal{R}} \int_{a^{-1}(R)} u^{*} \lambda .
$$

(b) The energies with and without $\varepsilon$ are related by

$$
\begin{gathered}
2 \varepsilon E_{\lambda}(f)-E_{\omega}(f) \leq E_{\lambda}^{\varepsilon}(f) \leq 2 \varepsilon E_{\lambda}(f)+E_{\omega}(f), \\
\frac{2 \varepsilon}{1+2 \varepsilon} E(f) \leq E^{\varepsilon}(f) \leq 2 \max (1, \varepsilon) E(f) .
\end{gathered}
$$

Proof.

(a) The orientation of $a^{-1}(R)$ as the boundary of the region $\{a \leq R\}$ means that a tangent vector $v$ to $a^{-1}(R)$ is orienting the level iff $d a(-j v)>0$. Since $u^{*} \lambda(v)=d a(-j v)$, this shows $\int_{a^{-1}(R)} u^{*} \lambda>0$. For the second statement, we compute for $\phi \in \mathcal{S}$ and regular values $R<S$ of $a$ :

$$
\begin{gathered}
\phi(S) \int_{a^{-1}(S)} u^{*} \lambda-\phi(R) \int_{a^{-1}(R)} u^{*} \lambda=\int_{a^{-1}([R, S])} f^{*} d(\phi(r) \lambda) \\
=\int_{a^{-1}([R, S])} f^{*}\left(\phi^{\prime}(r) d r \wedge \lambda\right)+\int_{a^{-1}([R, S])} f^{*}(\phi(r) d \lambda) .
\end{gathered}
$$

Since its integrand is non-negative, the first term in the last line is $\leq E_{\lambda}(f)$. The second term in the last line becomes arbitrarily small if we choose $R$ close to $S$. Taking $\phi(R)=0$ and $\phi(S)=1$, this shows $\int_{a^{-1}(R)} u^{*} \lambda \leq E_{\lambda}(f)$. The converse inequality follows from the following estimate for $\phi \in \mathcal{S}$ :

$$
\begin{aligned}
\int_{\Sigma} f^{*}\left(\phi^{\prime}(r) d r \wedge \lambda\right) & =\int_{\mathcal{R}} \phi^{\prime}(r)\left(\int_{a^{-1}(r)} u^{*} \lambda\right) d r \\
& \leq \sup _{R \in \mathcal{R}} \int_{a^{-1}(R)} u^{*} \lambda \int_{-\infty}^{\infty} \phi^{\prime}(r) d r \leq \sup _{R \in \mathcal{R}} \int_{a^{-1}(R)} u^{*} \lambda .
\end{aligned}
$$

(b) Write the $(\varepsilon, \lambda)$-energy as

$$
E_{\lambda}^{\varepsilon}(f)=\sup _{\phi \in \mathcal{S}_{\varepsilon}}\left(\int_{\Sigma} f^{*}\left(\phi^{\prime}(r) d r \wedge \lambda\right)+\int_{\Sigma} f^{*}(\phi(r) d \lambda)\right) .
$$

The absolute value of the second integral on the right-hand side is not bigger than $\int_{\Sigma} f^{*} \omega=E_{\omega}(f)$ by definition of $\varepsilon$ while the supremum 
of the first integral equals $2 \varepsilon E_{\lambda}(f)$. This shows the first statement $\left|E_{\lambda}^{\varepsilon}(f)-2 \varepsilon E_{\lambda}(f)\right| \leq E_{\omega}(f)$. Rewrite this as $2 \varepsilon E_{\lambda}(f) \leq E^{\varepsilon}(f)$ and $E^{\varepsilon}(f) \leq 2 \varepsilon E_{\lambda}(f)+2 E_{\omega}(f)$. The second statement follows from these inequalities:

$$
\begin{gathered}
(1+2 \varepsilon) E^{\varepsilon}(f) \geq E^{\varepsilon}(f)+2 \varepsilon E_{\omega}(f) \geq 2 \varepsilon E(f), \\
E^{\varepsilon}(f) \leq 2 \varepsilon E_{\lambda}(f)+2 E_{\omega}(f) \leq 2 \max (1, \varepsilon) E(f) .
\end{gathered}
$$

The following lemma gives a lower bound for the area of an arbitrary punctured holomorphic curve with no negative punctures.

Lemma 4.2. There is a number $\beta_{1}>0$, depending only on $(M, \omega, J)$, such that for any punctured holomorphic curve $f: \dot{\Sigma} \rightarrow \mathbb{R} \times M$ without negative or without positive punctures

$$
E_{\omega}(f)>\beta_{1} .
$$

Note that the statement includes $J$-holomorphic curves without any punctures at all and that $\beta_{1}$ does not depend on the number of punctures, the genus of $\Sigma$, nor the Hofer energy of $f$.

Proof. Assume that $f=(a, u)$ has no negative punctures. We apply the Monotonicity Lemma 3.17 to $\mathbb{R} \times M$ equipped with the product metric $\omega(\cdot, J \cdot)+d r^{2}+\lambda^{2}$, which is $J$-invariant by assumption. Pick any point $f(z)$ on the curve. Then the area of (a connected component of) the pre-image $C$ of a ball $B$ of radius $\rho<\varepsilon_{\mathrm{ML}}$ satisfies

$$
\int_{C}\left(u^{*} \omega+d a \wedge u^{*} \lambda\right) \geq C_{\mathrm{ML}} \rho^{2} .
$$

Let $\rho:=\min \left(\varepsilon, \varepsilon_{\mathrm{ML}}\right) / 4$. Pick a function $\phi: \mathbb{R} \rightarrow[-\varepsilon / 2,0]$ with $\phi^{\prime} \geq 0$ and $\phi(r) \rightarrow 0$ as $r \rightarrow \infty$ with $\phi^{\prime} \equiv 1$ on $[a(z)-\varepsilon / 4, a(z)+\varepsilon / 4]$. Then we have

$$
\begin{aligned}
E_{\omega}(f) & =\int_{\Sigma}\left(u^{*} \omega+f^{*} d(\phi \lambda)\right) \\
& \geq \int_{C}\left(u^{*} \omega+f^{*} d(\phi \lambda)\right) \\
& \geq \int_{C}\left(\frac{1}{2} u^{*} \omega+f^{*}(d \phi \wedge \lambda)\right) \\
& =\int_{C}\left(\frac{1}{2} u^{*} \omega+d a \wedge u^{*} \lambda\right) \\
& \geq C_{\mathrm{ML}} \rho^{2} / 2 .
\end{aligned}
$$

Here, in the first line, we have used $\int_{\Sigma} f^{*} d(\phi \lambda)=0$; in the second line the positivity of the integrand on $\Sigma$; in the third line the positivity of $f^{*}(\phi d \lambda)+$ $\frac{1}{2} u^{*} \omega$ which is due to $|\phi| \leq \varepsilon / 2$ and the definition of $\varepsilon$; in the fourth line 
$\phi^{\prime}(a) \equiv 1$ on $C$ which holds because $|a-a(z)|<\varepsilon / 4$ on $C$; in the last line the positivity of $d a \wedge u^{*} \lambda$.

The case that $f$ has no positive punctures is similar. Here we choose $\phi: \mathbb{R} \rightarrow[0, \varepsilon / 2]$.

4.2. Estimates for holomorphic cylinders. Set $S^{1}:=\mathbb{R} / \mathbb{Z}$, so an annulus $[R, S] \times S^{1}$ (with the standard conformal structure) has modulus $S-R$, cf. Section 3, Lemma 3.4.

Lemma 4.3. For every $J$-holomorphic cylinder $f=(a, u):[-L, L] \times S^{1} \rightarrow$ $\mathbb{R} \times M$ satisfying $E(f) \leq E_{0}$ and $E_{\omega}(f) \leq \beta_{1}$ and every $\delta>0$, all derivatives of $f$ (of order $\geq 1$ for a) on $[-L+\delta, L-\delta] \times S^{1}$ are bounded by constants depending only on $M, \omega, J, E_{0}$ and $\delta$.

Proof. By elliptic bootstrapping, it suffices to prove uniform gradient bounds. Suppose by contradiction that there is a sequence of $J$-holomorphic cylinders $f_{k}:\left[-L_{k}, L_{k}\right] \times S^{1} \rightarrow \mathbb{R} \times M$ satisfying $E\left(f_{k}\right) \leq E_{0}$ and $E_{\omega}\left(f_{k}\right) \leq \beta_{1}$ whose gradients are unbounded at points in $\left[-L_{k}+\delta, L_{k}-\delta\right]$. Then standard bubbling off analysis as in [7] (see also [3]) yields a nonconstant punctured $J$-holomorphic plane $f$ with $E(f) \leq E_{0}$ and $E_{\omega}(f) \leq \beta_{1}$. Hence the singularity at $\infty$ is either removable or gives rise to one (positive or negative) puncture. Hence Lemma 4.2 implies $E_{\omega}(f)>\beta_{1}$, which is a contradiction.

Recall next the asymptotic behavior of holomorphic curves with finite energy. The following result was proved in [7] in the Morse case and in $[\mathbf{2}, \mathbf{8}]$ in the Morse-Bott case.

Proposition 4.4. Let $f=(a, u): \mathbb{R}_{+} \times S^{1} \rightarrow \mathbb{R} \times M$ be a J-holomorphic half-cylinder of finite Hofer energy such that a is not bounded from above. Then there exists a closed Reeb orbit $\gamma$ of period $T>0$ and a constant $a_{0}$ such that

uniformly in $t$ with all derivatives.

$$
\left(a(s, t)-a_{0}-T s, u(s, t)\right) \underset{s \rightarrow \infty}{\longrightarrow}(0, \gamma(T t))
$$

The following proposition shows that the $M$-components of long holomorphic cylinders approach closed Reeb orbits. It was proved in [9] in the Morse case and in [3] (Proposition 5.7) in the Morse-Bott case. Let $d$ be any Riemannian distance on $M$.

Proposition 4.5. For all $E_{0}, \delta>0$, there exist $\beta, \ell>0$ (depending only on $M, \omega, J, E_{0}$ and $\left.\delta\right)$ such that for every $J$-holomorphic cylinder $f=(a, u)$ : $[-L, L] \times S^{1} \rightarrow \mathbb{R} \times M$ with $L>\ell$ satisfying $E(f) \leq E_{0}$ and $E_{\omega}(f) \leq \beta$, we have

$$
d(u(s, t), u(0, t))<\delta
$$

for all $(s, t) \in[-L+\ell, L-\ell] \times S^{1}$. 
The following lemma guarantees that the area of a $J$-holomorphic cylinder (a punctured holomorphic sphere with one positive and one negative puncture) cannot be arbitrarily small. It was proved in [3] (Lemma 11.8.). We include the proof here for sake of completeness.

Lemma 4.6. For every $E_{0}>0$, there exists a constant $\beta_{2}>0$ (depending only on $M, \omega, J$ and $\left.E_{0}\right)$ such that the area of every $J$-holomorphic cylinder $f: \mathbb{R} \times S^{1} \rightarrow \mathbb{R} \times M$ with $E(f) \leq E_{0}$ and $E_{\omega}(f)>0$ satisfies

$$
E_{\omega}(f)>\beta_{2} \text {. }
$$

Remark 4.7. If $\omega=d \lambda$ for a contact form $\lambda$, this statement follows immediately from discreteness of the action spectrum (which is an easy consequence of the Morse-Bott hypothesis). In the general case, discreteness of the values of $E_{\omega}$ still holds as a consequence of Gromov-Hofer compactness. However, as we want to use Lemma 4.6 in the proof of this compactness theorem, we cannot use discreteness here.

Proof. Assume on the contrary that there is a sequence $f_{n}=\left(a_{n}, u_{n}\right)$ : $\mathbb{R} \times S^{1} \rightarrow \mathbb{R} \times M$ of $J$-holomorphic cylinders with $E\left(f_{n}\right) \leq E_{0}$ and positive areas $0<E_{\omega}\left(f_{n}\right)=\delta_{n} \rightarrow 0$. Denote the asymptotic orbits of $f_{n}$ by $\gamma_{n}^{ \pm}$. By Proposition 4.4 , there exist $L_{n}>0$ such that

$$
\begin{gathered}
d\left(u_{n}(s, t), \gamma_{n}^{+}(t)\right)<\frac{1}{n} \quad \text { for } s \in\left[L_{n}, \infty\right), \\
d\left(u_{n}(s, t), \gamma_{n}^{-}(t)\right)<\frac{1}{n} \quad \text { for } s \in\left(-\infty,-L_{n}\right] .
\end{gathered}
$$

Let $\ell_{n}, \beta_{n}$ be the constant in Proposition 4.5 corresponding to $E_{0}, \delta=1 / n$. By relabeling the sequence $\left\{f_{n}\right\}$, we may assume that $\delta_{n}<\beta_{n}$. The maps $\left.f_{n}\right|_{\left[-L_{n}-\ell_{n}, L_{n}+\ell_{n}\right] \times S^{1}}$ have energy $\leq E_{0}$ and area $\leq \delta_{n}$. Hence, by Proposition 4.5,

$$
d\left(u_{n}(s, t), u_{n}(0, t)\right)<\frac{1}{n}
$$

for all $s \in\left[-L_{n}, L_{n}\right]$. It follows that

$$
d\left(u_{n}(s, t), \gamma_{n}^{ \pm}(t)\right)<\frac{4}{n}
$$

for all $s \in \mathbb{R}$, so $u_{n}$ stays in the $4 / n$-neighborhood of $\gamma^{ \pm}$in the loop space $\Lambda M$ of $M$ (equipped with the $C^{0}$-topology). In particular, $d\left(\gamma^{+}(t), \gamma^{-}(t)\right)<4 / n$ for all $t \in S^{1}$. By compactness of $M$ and the Morse-Bott assumption, there are only finitely many connected manifolds of closed Reeb orbits of $\lambda$-action smaller than $E_{0}$. Pick $n$ so large that the $4 / n$-neighborhoods of these manifolds in $\Lambda M$ are disjoint. Then the asymptotic orbits $\gamma_{n}^{ \pm}$must belong to the same connected manifold $\mathcal{C}$ of closed Reeb orbits. Moreover, the path $u_{n}: \mathbb{R} \rightarrow \Lambda M$ in the loop space stays in the $C / n$-neighborhood of $\mathcal{C}$ for a constant $C$ that only depends on the compact submanifold $\mathcal{C} \subset \Lambda M$. 
Therefore, for $n$ sufficiently large, we can project $u_{n}$ onto a smooth path $v_{n}: \mathbb{R} \rightarrow \mathcal{C}$ connecting $\gamma^{ \pm}$in the manifold $\mathcal{C}$. Since $v_{n}(s, \cdot)$ is a closed Reeb orbit for all $s$, we have $v_{n}^{*} \omega \equiv 0$ and thus $\int_{\mathbb{R} \times S^{1}} v_{n}^{*} \omega=0$. Since $\omega$ is closed and the cylinders $u_{n}, v_{n}: \mathbb{R} \times S^{1} \rightarrow M$ are homotopic with fixed loops at $\pm \infty$, it follows that

$$
\delta_{n}=E_{\omega}\left(f_{n}\right)=\int_{\mathbb{R} \times S^{1}} v_{n}^{*} \omega=0,
$$

contradicting the assumption $\delta_{n}>0$.

Corollary 4.8. Let $f: \mathbb{R} \times S^{1} \rightarrow \mathbb{R} \times M$ be a holomorphic map with Hofer energy $E(f)<E_{0}$ and action $E_{\omega}(f)<\beta_{1}$. Then $f$ must be constant or has exactly one positive and one negative puncture. Moreover, if $E_{\omega}(f)<\beta_{2}, f$ is a cylinder over a closed Reeb orbit.

Fix a number $E_{0}<\infty$. We will study $J$-holomorphic curves (in fact, cylinders $) f:(\Sigma, j) \rightarrow(\mathbb{R} \times M, J)$ satisfying the following hypothesis:

$$
E(f) \leq E_{0}, \quad E_{\omega}(f) \leq \beta_{0}:=\min \left(\beta_{1}, \beta_{2}\right),
$$

where $\beta_{1}, \beta_{2}$ are the constants from Lemma 4.2 and Lemma 4.6, respectively.

We first note a sharpening of Proposition 4.5.

Corollary 4.9. For all $E_{0}, \delta>0$, there exists an $\ell>0$ (depending only on $M, \omega, J, E_{0}$ and $\left.\delta\right)$ such that for every $J$-holomorphic cylinder $f=(a, u)$ : $[-L, L] \times S^{1} \rightarrow \mathbb{R} \times M$ with $L>\ell$ satisfying hypothesis (B1), we have

$$
d(u(s, t), u(0, t))<\delta
$$

for all $(s, t) \in[-L+\ell, L-\ell] \times S^{1}$.

Proof. Assume that, on the contrary (after replacing $\ell$ by $2 \ell$ ), there is a $\bar{\delta}>0$ such that for any $\ell>0$, there is an $L>2 \ell$ and a $J$-holomorphic cylinder $f=(a, u):[-L, L] \times S^{1} \rightarrow \mathbb{R} \times M$ with $E(f) \leq E_{0}$ and $E_{\omega}(f) \leq \beta_{0}$ such that $d(u(s, t), u(0, t)) \geq \bar{\delta}$ for some $(s, t) \in[-L+2 \ell, L-2 \ell] \times S^{1}$. Let $\bar{\beta}>0$ and $\bar{\ell}>0$ be the constants corresponding to $\bar{\delta}$ and $E_{0}$ in Proposition 4.5. Applying Proposition 4.5 to the restriction $g:=\left.f\right|_{[-L+\ell, L-\ell] \times S^{1}}$ for $\ell \geq \bar{\ell}$, we conclude that $E_{\omega}(g)>\bar{\beta}$. Write $g=(b, v):[-c, c] \times S^{1} \rightarrow \mathbb{R} \times M$ with $c:=L-\ell$.

Now we argue as in the proof of Lemma 4.6. By the Morse-Bott assumption, there are finitely many connected manifolds of closed Reeb orbits of $\lambda$-action smaller than $2 E_{0}$. Fix $\rho>0$ so small that the $\rho$-neighborhoods of these manifolds in the loops space $\Lambda M$ (with the $C^{0}$-topology) are disjoint. Suppose that $v(s, \cdot)$ is $\rho$-close to a closed Reeb orbit for all $s \in[-c, c]$. Since $E(f) \leq E_{0}$, this implies that $v(s, \cdot)$ is $\rho$-close to one connected manifold $\mathcal{C}$ of Reeb orbits for all $s \in[-c, c]$. As in the proof of Lemma 4.6, we project $v$ onto a path $\bar{v}$ in $\mathcal{C}$. Let $v_{ \pm}$be the shortest path connecting $v( \pm c, \cdot)$ to 
its projection $\bar{v}( \pm c, \cdot)$. Then $v, \bar{v}$ and $v^{ \pm}$form a null-homotopic loop $\tilde{v}$ in $\Lambda M$, so $\int \tilde{v}^{*} \omega=0$. Now $\int \bar{v}^{*} \omega=0$ because $\bar{v}$ consists of Reeb orbits, and $\int v_{ \pm}^{*} \omega<C \rho$ for a constant $C$ only depending on $\mathcal{C}$ because $\omega=d \lambda_{ \pm}$is exact in the $\rho$-neighborhood of $\bar{v}( \pm c, \cdot)$. So we conclude that $\int v^{*} \omega<2 C \rho$ if $v(s, \cdot)$ is $\rho$-close to a closed Reeb orbit for all $s \in[-c, c]$. For $\rho<\bar{\beta} / 2 C$, this implies that there exists a $\bar{s} \in[-L+\ell, L-\ell]$ such that $u(\bar{s},$.$) is not$ $\rho$-close any closed Reeb orbit.

Now pick a sequence $\ell_{n} \rightarrow \infty$ and define the sequence of $J$-holomorphic cylinders $f_{n}=\left(a_{n}, u_{n}\right):\left[-L_{n}, L_{n}\right] \times S^{1} \rightarrow \mathbb{R} \times M$ as above, with $L_{n}>2 \ell_{n}$. Thus $E\left(f_{n}\right) \leq E_{0}, E_{\omega}\left(f_{n}\right) \leq \beta_{0}$, and there exist $\bar{s}_{n} \in\left[-L_{n}+\ell_{n}, L_{n}-\ell_{n}\right]$ such that $u_{n}\left(\bar{s}_{n},.\right)$ is not $\rho$-close to any closed Reeb orbit (with $\rho>0$ fixed as above). Set $g_{n}:=\left(b_{n}, v_{n}\right):\left[-\ell_{n}, \ell_{n}\right] \times S^{1} \rightarrow \mathbb{R} \times M$ with

$$
v_{n}(s, t):=u_{n}\left(s+\bar{s}_{n}, t\right), \quad b_{n}(s, t):=a_{n}\left(s+\bar{s}_{n}, t\right)-a_{n}\left(\bar{s}_{n}, t\right) .
$$

By Lemma 4.3, all derivatives of $g_{n}$ are uniformly bounded. Hence a subsequence of $g_{n}$ converges in $C_{\text {loc }}^{\infty}$ to an infinite holomorphic cylinder $g=(b, v): \mathbb{R} \times S^{1} \rightarrow \mathbb{R} \times M$ with $E(g) \leq E_{0}$ and $E_{\omega}(g) \leq \beta_{0}$. Since $\beta_{0} \leq \beta_{2}$, Lemma 4.6 implies $E_{\omega}(g)=0$, so $g$ is a cylinder over a closed Reeb orbit. On the other hand, by construction of $g_{n}$ and $C_{\text {loc }}^{\infty}$-convergence, $v(0, \cdot)$ is not $\rho$-close to a closed Reeb orbit. This contradiction concludes the proof.

The next lemma describes the $\mathbb{R}$-components of long holomorphic cylinders. Notice that the action of a closed Reeb orbit cannot be arbitrarily small:

$$
\int_{\gamma} \lambda>\delta_{0}>0
$$

for any closed $R$-orbit $\gamma$.

Lemma 4.10. There exists an $\ell>0$ (depending only on $M, \omega, J$ and $E_{0}$ ) such that for every $J$-holomorphic cylinder $f=(a, u):[-L, L] \times S^{1} \rightarrow \mathbb{R} \times M$ with $L>\ell$ satisfying hypothesis (B1),

$$
\frac{\partial a}{\partial s}(s, t)>\delta_{0}>0
$$

for all $(s, t) \in[-L+\ell, L-\ell] \times S^{1}$.

Proof. Suppose not. Then there exist sequences $L_{k}>\ell_{k} \rightarrow \infty$ and $J$ holomorphic cylinders $f_{k}=\left(a_{k}, u_{k}\right):\left[-L_{k}, L_{k}\right] \times S^{1} \rightarrow \mathbb{R} \times M$ satisfying hypothesis (B1) such that

$$
\frac{\partial a_{k}}{\partial s}\left(s_{k}, t_{k}\right) \leq \delta_{0}
$$


for some $\left(s_{k}, t_{k}\right) \in\left[-L_{k}+\ell_{k}, L_{k}-\ell_{k}\right] \times S^{1}$. Consider the shifted maps $g_{k}=\left(b_{k}, v_{k}\right):\left[-\ell_{k}, \ell_{k}\right] \times S^{1} \rightarrow \mathbb{R} \times M$,

$$
g_{k}(s, t):=f_{k}\left(s+s_{k}, t+t_{k}\right)-\left(a_{k}\left(s_{k}, t_{k}\right), 0\right) .
$$

By the $C^{\infty}$-bounds in Lemma 4.3 and the Arzela-Ascoli theorem, a subsequence of $g_{k}$ converges in $C_{\mathrm{loc}}^{\infty}$ to an infinite holomorphic cylinder $g=$ $(b, v): \mathbb{R} \times S^{1} \rightarrow \mathbb{R} \times M$ of energy $E(g) \leq E_{0}$. By Proposition $4.4, g$ is asymptotic at its punctures to closed Reeb orbits $\gamma^{-}, \gamma^{+}$. By construction, we have $E(g) \leq E_{0}$ and $E_{\omega}(g) \leq \beta_{0}$. By Lemma 4.6, $g$ has area zero and is hence a cylinder over a closed Reeb orbit $\gamma$ of action $T>\delta_{0}$. So we have $\frac{\partial b}{\partial s}(0,0)=T>\delta_{0}$. On the other hand, $\frac{\partial b_{k}}{\partial s}(0,0) \leq \delta_{0}$ for all $k$ implies $\frac{\partial b}{\partial s}(0,0) \leq \delta_{0}$ and we have a contradiction.

4.3. $C_{\mathrm{loc}}^{\infty}$-convergence. Consider now a sequence of $J$-holomorphic cylinders

$$
f_{k}=\left(a_{k}, u_{k}\right):\left[-L_{k}-1, L_{k}+1\right] \times S^{1} \rightarrow \mathbb{R} \times M
$$

satisfying hypothesis (B1) with uniform constants $E_{0}, \beta_{0}$. Set

$$
r_{k}^{-}:=\inf _{t} a_{k}\left(-L_{k}, t\right), \quad r_{k}^{+}:=\sup _{t} a_{k}\left(L_{k}, t\right),
$$

and impose the hypothesis

$$
L_{k} \underset{k \rightarrow \infty}{\longrightarrow} \infty, \quad r_{k}^{+}-r_{k}^{-} \underset{k \rightarrow \infty}{\longrightarrow} \infty .
$$

Define the shifted maps

$$
\begin{array}{ll}
f_{k}^{-}(s, t):=f_{k}\left(s-L_{k}, t\right)-\left(r_{k}^{-}, 0\right), & s \in\left[0,2 L_{k}\right], \\
f_{k}^{+}(s, t):=f_{k}\left(s+L_{k}, t\right)-\left(r_{k}^{+}, 0\right), & s \in\left[-2 L_{k}, 0\right] .
\end{array}
$$

Lemma 4.11. A subsequence of the shifted maps $f_{k}^{-}:\left[0,2 L_{k}\right] \times S^{1} \rightarrow$ $\mathbb{R} \times M$ converges in $C_{\mathrm{loc}}^{\infty}$ on $[0, \infty) \times S^{1}$ to a holomorphic half-cylinder $f^{-}$: $[0, \infty) \times S^{1} \rightarrow \mathbb{R} \times M$. Similarly, a subsequence of the $f_{k}^{+}:\left[-2 L_{k}, 0\right] \times$ $S^{1} \rightarrow \mathbb{R} \times M$ converges in $C_{\mathrm{loc}}^{\infty}$ on $(-\infty, 0] \times S^{1}$ to a holomorphic halfcylinder $f^{+}:(-\infty, 0] \times S^{1} \rightarrow \mathbb{R} \times M$. Moreover, $f^{-}$and $f^{+}$are positively resp. negatively asymptotic to closed Reeb orbits $\gamma^{-}, \gamma^{+}$of length not greater than $E_{0}$.

Proof. The uniform bounds on derivatives in Lemma 4.3 and the ArzelaAscoli theorem imply $C_{\text {loc }}^{\infty}$-convergence of subsequences $f_{k}^{-} \rightarrow f^{-}$and $f_{k}^{+} \rightarrow f^{+}$. The limit maps have Hofer energy at most $E_{0}$. Moreover, hypothesis (B2) implies that $a^{-}$is not bounded from above and $a^{+}$is not bounded from below. So, by Proposition 4.4, $f^{-}, f^{+}$are asymptotic to closed Reeb orbits $\gamma^{-}, \gamma^{+}$. By Lemma 4.1, we have $\int_{\gamma^{-}} \lambda, \int_{\gamma^{+}} \lambda \leq E_{0}$.

The following two lemmas control the maps $f_{k}$ over the middle parts of the cylinders. 
Lemma 4.12. The asymptotic orbits agree: $\gamma^{-}=\gamma^{+}=: \gamma$. Moreover, for every $\delta>0$, there exist a constant $L>0$ (depending only on $M, \omega, J, E_{0}$ and $\delta$ ) such that for all sufficiently large $k$ and for all $(s, t) \in\left[-L_{k}+L, L_{k}-\right.$ $L] \times S^{1}$,

$$
d\left(u_{k}(s, t), \gamma(t)\right) \leq \delta .
$$

Proof. Let $\delta>0$ be given. By Corollary 4.9, there exists an $L>0$ (depending only on $M, \omega, J, E_{0}$ and $\left.\delta\right)$ such that $d\left(u_{k}(s, t), u_{k}(0, t)\right)<\delta$ for all $(s, t) \in\left[-L_{k}+L, L_{k}-L\right] \times S^{1}$ and all sufficiently large $k$. By the asymptotics, for $s \geq L$ sufficiently large, $u^{-}(s, t)$ is $\delta$-close to $\gamma_{-}(t)$ for all $t \in S^{1}$. By the $C_{\text {loc }}^{\infty}$-convergence of $u_{k}^{-}$, for $k$ sufficiently large, $u_{k}\left(-L_{k}+s, t\right)$ is $\delta$ close to $u^{-}(s, t)$ for all $t \in S^{1}$. Thus $u_{k}(0, t)$ is $3 \delta$-close to $\gamma^{-}(t)$ for all $t \in S^{1}$. Similarly, $u_{k}(0, t)$ is $3 \delta$-close to $\gamma^{+}(t)$ for all $t \in S^{1}$. Hence $\gamma^{-}(t)$ is $6 \delta$-close to $\gamma^{+}(t)$ for all $t \in S^{1}$. Since this holds for all $\delta$, we conclude $\gamma^{-}=\gamma^{+}=: \gamma$. Moreover, the preceding argument shows that for $k$ sufficiently large, $u_{k}(s, t)$ is $4 \delta$-close to $\gamma(t)$ for all $(s, t) \in\left[-L_{k}+L, L_{k}-L\right] \times S^{1}$. Now replace $4 \delta$ by $\delta$.

Lemma 4.13. For every $R>0$, there exist a constant $L>0$ (depending on the sequence $\left(f_{k}\right)$ and $\left.R\right)$ such that for all sufficiently large $k$ and for all $(s, t) \in\left[-L_{k}+L, L_{k}-L\right] \times S^{1}$,

$$
a_{k}(s, t) \in\left[r_{k}^{-}+R, r_{k}^{+}-R\right] .
$$

Proof. By Lemma 4.10, there exists an $\ell>0$ such that $\frac{\partial a_{k}}{\partial s}(s, t)>0$ for all $(s, t) \in\left[-L_{k}+\ell, L_{k}-\ell\right] \times S^{1}$ and sufficiently large $k$. By the asymptotics, for $L \geq \ell$ sufficiently large, $a^{-}(L, t) \geq R+1$ for all $t \in S^{1}$. By the $C_{\text {loc }}^{\infty}$-convergence of $a_{k}^{-}$, for $k$ sufficiently large, $a_{k}\left(-L_{k}+L, t\right) \geq r_{k}^{-}+R$ for all $t \in S^{1}$. Similarly, $a_{k}\left(L_{k}-L, t\right) \leq r_{k}^{+}-R$ for all $t \in S^{1}$. Now the statement follows from the monotonicity $\frac{\partial a_{k}}{\partial s}(s, t)>0$ for $(s, t) \in\left[-L_{k}+L, L_{k}-L\right] \times S^{1}$.

4.4. Uniform convergence. Let $f_{k}=\left(a_{k}, u_{k}\right):\left[-L_{k}-1, L_{k}+1\right] \times S^{1} \rightarrow$ $\mathbb{R} \times M$ and $f^{ \pm}=\left(a^{ \pm}, u^{ \pm}\right)$be as above. That is, $f_{k}$ satisfies (B1 and B2) and $f^{ \pm}$are the limit maps provided by Lemma 4.11. Consider a sequence of increasing diffeomorphisms $\theta_{k}:\left[-L_{k}, L_{k}\right] \rightarrow[-1,1]$ and define the shifted maps

$$
\begin{array}{ll}
\theta_{k}^{-}(s):=\theta_{k}\left(s-L_{k}\right), & s \in\left[0,2 L_{k}\right], \\
\theta_{k}^{+}(s):=\theta_{k}\left(s+L_{k}\right), & s \in\left[-2 L_{k}, 0\right] .
\end{array}
$$

We impose the following hypothesis on the sequence $\theta_{k}$ :

$$
\theta_{k}^{-} \underset{k \rightarrow \infty}{\longrightarrow} \theta^{-}:[0, \infty) \longrightarrow[-1,0), \quad \theta_{k}^{+} \underset{k \rightarrow \infty}{\longrightarrow} \theta^{+}:(-\infty, 0] \rightarrow(0,1],
$$

where the convergence is in $C_{\mathrm{loc}}^{\infty}$ on $[0, \infty)$ resp. $(-\infty, 0]$ and the maps $\theta^{-}, \theta^{+}$ are diffeomorphisms. 
Remark 4.14. Such a sequence clearly exists: Pick arbitrary diffeomorphisms $\theta^{ \pm}$and let $\theta_{k}$ be any diffeomorphism with $\theta_{k}(s)=\theta^{-}\left(s+L_{k}\right)$ for $s \in\left[-L_{k},-1\right]$ and $\theta_{k}(s)=\theta^{+}\left(s-L_{k}\right)$ for $s \in\left[1, L_{k}\right]$.

Define maps

$$
\begin{aligned}
& g_{k}(s, t):=f_{k}\left(\theta_{k}^{-1}(s), t\right), \quad s \in[-1,1], \\
& g_{k}^{-}(s, t):=f_{k}^{-}\left(\left(\theta_{k}^{-}\right)^{-1}(s), t\right), \quad s \in[-1,1], \\
& g_{k}^{+}(s, t):=f_{k}^{+}\left(\left(\theta_{k}^{+}\right)^{-1}(s), t\right), \quad s \in[-1,1], \\
& g^{-}(s, t):=f^{-}\left(\left(\theta^{-}\right)^{-1}(s), t\right), \quad s \in[-1,0), \\
& g^{+}(s, t):=f^{+}\left(\left(\theta^{+}\right)^{-1}(s), t\right), \quad s \in(0,1] .
\end{aligned}
$$

Lemma 4.15. We have $C_{\mathrm{loc}}^{\infty}$-convergence $g_{k}^{-} \rightarrow g^{-}$on $[-1,0) \times S^{1}$ and $g_{k}^{+} \rightarrow g^{+}$on $(0,1] \times S^{1}$.

Proof. Note that the inverse maps to $\theta_{k}^{ \pm}$are given by

$$
\left(\theta_{k}^{-}\right)^{-1}(s)=\theta_{k}^{-1}(s)+L_{k}, \quad\left(\theta_{k}^{+}\right)^{-1}(s)=\theta_{k}^{-1}(s)-L_{k} .
$$

The convergence assumption (B3) implies $\theta_{k}^{-1}(0)+L_{k} \rightarrow \infty$ and $\theta_{k}^{-1}(0)-$ $L_{k} \rightarrow-\infty$. Moreover, the restrictions $\left(\theta_{k}^{-}\right)^{-1}:[-1,0) \rightarrow\left[0, \theta_{k}^{-1}(0)+L_{k}\right)$ and $\left(\theta_{k}^{+}\right)^{-1}:(0,1] \rightarrow\left(\theta_{k}^{-1}(0)-L_{k}, 0\right]$ converge in $C_{\text {loc }}^{\infty}$ to $\left(\theta^{-}\right)^{-1}$ resp. $\left(\theta^{+}\right)^{-1}$. Therefore, the maps $f_{k}^{-}\left(\left(\theta_{k}^{-}\right)^{-1}(s), t\right)$ converge to $g^{-}$in $C_{\text {loc }}^{\infty}$ on $[-1,0) \times S^{1}$ and similarly for $g^{+}$.

Write $g_{k}=\left(b_{k}, v_{k}\right)$ and $g^{ \pm}=\left(b^{ \pm}, v^{ \pm}\right)$. By the asymptotics of $f^{ \pm}$, the $M$-components $v^{ \pm}$fit together to a continuous map (which is smooth in $t$ )

$$
v(s, t):= \begin{cases}v^{-}(s, t) & : s \in[-1,0), \\ \gamma(t) & : s=0, \\ v^{+}(s, t) & : s \in(0,1] .\end{cases}
$$

Lemma 4.16. The maps $v_{k}$ converge to $v$ uniformly on $[-1,1] \times S^{1}$.

Proof. Let $\delta>0$ be given. In view of the continuity of $v$ and the $C_{\mathrm{loc}^{-}}^{\infty}$ convergence in Lemma 4.15, it suffices to find $\sigma>0$ and $K \in \mathbb{N}$ such that

$$
d\left(v_{k}(s, t), \gamma(t)\right) \leq \delta
$$

for all $(s, t) \in[-\sigma, \sigma] \times S^{1}$ and $k \geq K$. By Lemma 4.12, there exist $K \in \mathbb{N}$ and $L>0$ such that for all $k \geq K$ and for all $(s, t) \in\left[-L_{k}+L, L_{k}-L\right] \times S^{1}$,

$$
d\left(u_{k}(s, t), \gamma(t)\right) \leq \delta .
$$


Recall that $\left(\theta^{-}\right)^{-1}$ maps $[-1,0)$ diffeomorphically onto $[0, \infty)$. Thus we find a $\sigma>0$ such that $\left(\theta^{-}\right)^{-1}(-\sigma) \geq L+1$. By the $C_{\text {loc }}^{\infty}$-convergence, we obtain

$$
\theta_{k}^{-1}(-\sigma)+L_{k}=\left(\theta_{k}^{-}\right)^{-1}(-\sigma) \geq L
$$

for $k$ sufficiently large, hence $\theta_{k}^{-1}(-\sigma) \geq-L_{k}+L$. Similarly, we can achieve $\theta_{k}^{-1}(\sigma) \leq L_{k}-L$ and, therefore, by monotonicity of $\theta_{k}$,

$$
\theta_{k}^{-1}([-\sigma, \sigma]) \subset\left[-L_{k}+L, L_{k}-L\right] .
$$

Then for $s \in[-\sigma, \sigma]$ and $k$ sufficiently large,

$$
d\left(v_{k}(s, t), \gamma(t)\right)=d\left(u_{k}\left(\theta_{k}^{-1}(s), t\right), \gamma(t)\right) \leq \delta .
$$

The following lemma asserts a kind of uniform convergence of the $\mathbb{R}$ components.

Lemma 4.17. For every $R>0$, there exist $\rho>0$ and $K \in \mathbb{N}$ such that $b_{k}(s, t) \in\left[r_{k}^{-}+R, r_{k}^{+}-R\right]$ for all $k \geq K$ and $(s, t) \in[-\rho, \rho] \times S^{1}$.

Proof. Let $R>0$ be given. By Lemma 4.13, there exists $L>0$ such that $a_{k}(s, t) \in\left[r_{k}^{-}+R, r_{k}^{+}-R\right]$ for all sufficiently large $k$ and $(s, t) \in\left[-L_{k}+\right.$ $\left.L, L_{k}-L\right] \times S^{1}$. As in the proof of Lemma 4.16, we find a $\rho>0$ such that

$$
\theta_{k}^{-1}([-\rho, \rho]) \subset\left[-L_{k}+L, L_{k}-L\right]
$$

for sufficiently large $k$. Then for $s \in[-\rho, \rho]$ and $k$ sufficiently large,

$$
b_{k}(s, t)=a_{k}\left(\theta_{k}^{-1}(s), t\right) \in\left[r_{k}^{-}+R, r_{k}^{+}-R\right] .
$$

Lemmas $4.15-4.17$ yield the main result of this section:

Theorem 4.18. Let $f_{k}:\left[-L_{k}-1, L_{k}+1\right] \times S^{1} \rightarrow \mathbb{R} \times M$ be a sequence of $J$-holomorphic cylinders satisfying hypotheses (B1) and (B2) with constants independent of $k$. Let $\theta_{k}:\left[-L_{k}, L_{k}\right] \rightarrow[-1,1]$ be a sequence of diffeomorphisms satisfying hypothesis (B3). Define the maps $g_{k}=\left(b_{k}, v_{k}\right), g^{ \pm}=$ $\left(b^{ \pm}, v^{ \pm}\right)$and $v$ as above. Then for a subsequence of $g_{k}$, the following holds:

(i) $g_{k}^{-} \rightarrow g^{-}$on $[-1,0) \times S^{1}$ and $g_{k}^{+} \rightarrow g^{+}$on $(0,1] \times S^{1}$ in $C_{\mathrm{loc}}^{\infty}$.

(ii) $v_{k} \rightarrow v$ uniformly on $[-1,1] \times S^{1}$.

(iii) For every $R>0$, there exist $\rho>0$ and $K \in \mathbb{N}$ such that $b_{k}(s, t) \in$ $\left[r_{k}^{-}+R, r_{k}^{+}-R\right]$ for all $k \geq K$ and $(s, t) \in[-\rho, \rho] \times S^{1}$.

4.5. Reformulation. We have stated Theorem 4.18 in the form it is needed in the proof of Theorem 2.9. For the sake of aesthetics, we will reformulate it in a more symmetric form. Recall that by hypothesis (B2), the numbers $r_{k}^{ \pm}$ satisfy $r_{k}^{+}-r_{k}^{-} \rightarrow \infty$ as $k \rightarrow \infty$. Moreover, by Lemma 4.11, there exists an $R_{0}>0$ such that $a_{k}(s, t) \in\left[r_{k}^{-}-R_{0}, r_{k}^{+}+R_{0}\right]$ for all $(s, t) \in\left[-L_{k}, L_{k}\right] \times S^{1}$. 
Consider a sequence of increasing diffeomorphisms $\Theta_{k}:\left[r_{k}^{-}-R_{0}, r_{k}^{+}+R_{0}\right] \rightarrow$ $[-1,1]$ and define the shifted maps

$$
\begin{array}{ll}
\Theta_{k}^{-}(s):=\Theta_{k}\left(s+r_{k}^{-}\right), & s \in\left[-R_{0}, r_{k}^{+}-r_{k}^{-}+R_{0}\right], \\
\Theta_{k}^{+}(s):=\Theta_{k}\left(s+r_{k}^{+}\right), & s \in\left[r_{k}^{-}-r_{k}^{+}-R_{0}, R_{0}\right] .
\end{array}
$$

We impose the following hypothesis on the sequence $\Theta_{k}$ :

$$
\Theta_{k}^{-} \underset{k \rightarrow \infty}{\longrightarrow} \Theta^{-}:\left[-R_{0}, \infty\right) \rightarrow[-1,0), \quad \Theta_{k}^{+} \underset{k \rightarrow \infty}{\longrightarrow} \Theta^{+}:\left(-\infty, R_{0}\right] \rightarrow(0,1],
$$

where the convergence is in $C_{\mathrm{loc}}^{\infty}$ on $\left[-R_{0}, \infty\right)$ resp. $\left(-\infty, R_{0}\right]$ and the maps $\Theta^{-}, \Theta^{+}$are diffeomorphisms. By the asymptotics of $f^{ \pm}$, the maps $\Theta^{ \pm} \circ b^{ \pm}$ fit together to a continuous map (which is smooth in $t$ )

$$
c(s, t):= \begin{cases}\Theta^{-} \circ b^{-}(s, t) & : s \in[-1,0), \\ 0 & : s=0, \\ \Theta^{+} \circ b^{+}(s, t) & : s \in(0,1] .\end{cases}
$$

Corollary 4.19. Let $f_{k}:\left[-L_{k}-1, L_{k}+1\right] \times S^{1} \rightarrow \mathbb{R} \times M$ be a sequence of $J$-holomorphic cylinders satisfying hypotheses (B1) and (B2) with constants independent of $k$. Let $\theta_{k}:\left[-L_{k}, L_{k}\right] \rightarrow[-1,1]$ and $\Theta_{k}:\left[r_{k}^{-}, r_{k}^{+}\right] \rightarrow[-1,1]$ be sequences of diffeomorphisms satisfying hypotheses (B3) and (B4), respectively. Define the maps

$$
\begin{gathered}
h_{k}:=\left(\Theta_{k} \circ b_{k}, v_{k}\right):[-1,1] \times S^{1} \longrightarrow[-1,1] \times M, \\
h:=(c, v):[-1,1] \times S^{1} \longrightarrow[-1,1] \times M
\end{gathered}
$$

as above. Then a subsequence of $h_{k}$ converges to $h$ in $C_{\mathrm{loc}}^{\infty}$ on $([-1,1] \backslash\{0\}) \times$ $S^{1}$ and uniformly on $[-1,1] \times S^{1}$.

Proof. Since

$$
\Theta_{k}^{-} \circ b_{k}^{-}=\Theta_{k} \circ b_{k}=\Theta_{k}^{+} \circ b_{k}^{+},
$$

the $C_{\mathrm{loc}}^{\infty}$-convergence follows from Lemma 4.15 and hypothesis (B4). For the uniform convergence, let $\delta>0$ be given. In view of the continuity of $c$ and the $C_{\text {loc }}^{\infty}$-convergence, it suffices to find $\rho>0$ and $K \in \mathbb{N}$ such that

$$
\left|\Theta_{k} \circ b_{k}(s, t)\right| \leq \delta
$$

for all $(s, t) \in[-\rho, \rho] \times S^{1}$ and $k \geq K$. Recall that $\Theta^{-}$maps $\left[-R_{0}, \infty\right)$ diffeomorphically onto $[-1,0)$. Thus we find an $R>0$ such that $\Theta^{-}(R) \geq$ $-\delta / 2$. By the $C_{\text {loc }}^{\infty}$-convergence, we obtain

$$
\Theta_{k}\left(r_{k}^{-}+R\right)=\Theta_{k}^{-}(R) \geq-\delta
$$

for $k$ sufficiently large. Similarly, we can achieve $\Theta_{k}\left(r_{k}^{+}-R\right) \leq \delta$ and, therefore, by monotonicity of $\Theta_{k}$,

$$
\Theta_{k}\left(\left[r_{k}^{-}+R, r_{k}^{+}-R\right]\right) \subset[-\delta, \delta] .
$$


By Lemma 4.17, there exists a $\rho>0$ such that $b_{k}(s, t) \in\left[r_{k}^{-}+R, r_{k}^{+}-R\right]$ for all sufficiently large $k$ and $(s, t) \in[-\rho, \rho] \times S^{1}$. Hence

$$
\Theta_{k} \circ b_{k}\left([-\rho, \rho] \times S^{1}\right) \subset[-\delta, \delta] .
$$

\subsection{Estimates on the conformal modulus}

Lemma 4.20. Denote by $A_{L}:=[0, L] \times S^{1}$ the annulus of conformal modulus L. Let $f=(a, u): A_{L} \rightarrow \mathbb{R} \times M$ be a J-holomorphic map with boundary conditions $a(0, t)=R$ and $a(L, t)=S$. Then

$$
\frac{S-R}{L} \leq \int_{\{L\} \times S^{1}} u^{*} \lambda+\frac{1}{\varepsilon} \int_{A_{L}} u^{*} \omega \leq \frac{1}{\varepsilon} E(f),
$$

where $\varepsilon>0$ is the constant from the beginning of this section. The first inequality is an equality if and only if $u^{*} \omega \equiv 0$.

Proof. Recall that in coordinates $(s, t)$ on the annulus, $J$-holomorphicity of $f$ is equivalent to

$$
a_{s}=\lambda\left(u_{t}\right), \quad a_{t}=-\lambda\left(u_{s}\right), \quad \pi u_{s}+J \pi u_{t}=0 .
$$

We compute

$$
\begin{aligned}
\int_{A_{L}} d s \wedge u^{*} \lambda & =\int_{0}^{L} d s \int_{\{s\} \times S^{1}} u^{*} \lambda=\int_{0}^{L} d s \int_{\{s\} \times S^{1}} a_{s}(s, t) d t \\
& =\int_{0}^{L} d s \frac{d}{d s} \int_{\{s\} \times S^{1}} a(s, t) d t=\int_{S^{1}}(a(L, t)-a(0, t)) d t \\
& =S-R .
\end{aligned}
$$

Now we use the hypothesis $u^{*}(\varepsilon d \lambda+\omega) \geq 0$ to obtain

$$
\begin{aligned}
\int_{A_{L}} d s \wedge u^{*} \lambda & =\int_{A_{L}} d\left(s u^{*} \lambda\right)-\int_{A_{L}} s u^{*} d \lambda \\
& \leq \int_{A_{L}} d\left(s u^{*} \lambda\right)+\frac{L}{\varepsilon} \int_{A_{L}} u^{*} \omega \\
& =L\left(\int_{\{L\} \times S^{1}} u^{*} \lambda+\frac{1}{\varepsilon} \int_{A_{L}} u^{*} \omega\right) \\
& \leq \frac{L E(f)}{\varepsilon},
\end{aligned}
$$

where the last inequality follows from Lemma 4.1. The last statement of the lemma holds since $u^{*}(\varepsilon d \lambda+\omega)=0$ only if $u^{*} \omega=0$.

Corollary 4.21. Let $f=(a, u): A_{L} \rightarrow \mathbb{R} \times M$ be a J-holomorphic map such that $\sup _{t} a(0, t)<\inf _{t} a(L, t)$. Then

$$
\inf _{t} a(L, t)-\sup _{t} a(0, t) \leq L\left(\int_{\{L\} \times S^{1}} u^{*} \lambda+\frac{1}{\varepsilon} \int_{A_{L}} u^{*} \omega\right) \leq \frac{L E(f)}{\varepsilon} .
$$


Proof. Pick $\varepsilon_{1}>0$ and regular levels $R, S$ of $a$ such that

$$
\sup _{t} a(0, t)<R<\sup _{t} a(0, t)+\varepsilon_{1}<\inf _{t} a(L, t)-\varepsilon_{1}<S<\inf _{t} a(L, t) .
$$

Then there exists a component $\gamma_{R}$ of $a^{-1}(R)$ separating $\{0\} \times S^{1}$ from $\{L\} \times$ $S^{1}$. (Otherwise we could connect $\{0\} \times S^{1}$ to $\{L\} \times S^{1}$ in $A_{L} \backslash a^{-1}(R)$, contradicting the hypothesis.) Similarly, there exists a component $\gamma_{S}$ of $a^{-1}(S)$ separating $\gamma_{R}$ from $\{L\} \times S^{1}$. Since $\gamma_{R}, \gamma_{S}$ are disjoint embedded loops isotopic to $\{0\} \times S^{1}$, they bound an annulus $A^{\prime} \subset A_{L}$ of modulus $L^{\prime} \leq L$ (see Section 3). Now apply Lemma 4.20 to $A^{\prime}$ to obtain

$$
\begin{gathered}
-2 \varepsilon_{1}+\inf _{t} a(L, t)-\sup _{t} a(0, t) \leq S-R \\
\leq L^{\prime}\left(\int_{\gamma_{S}} u^{*} \lambda+\frac{1}{\varepsilon} \int_{A^{\prime}} u^{*} \omega\right) \leq L\left(\int_{\{L\} \times S^{1}} u^{*} \lambda+\frac{1}{\varepsilon} \int_{A_{L}} u^{*} \omega\right) .
\end{gathered}
$$

Here the last inequality follows from nonnegativity of $u^{*}(\varepsilon d \lambda+\omega)$. As $\varepsilon_{1} \rightarrow 0$, the result follows.

The following lemma will be used in the proof of Theorem 2.9 to verify the hypotheses of Theorem 4.18 .

Lemma 4.22. Let $f=(a, u):[-L-1, L+1] \times S^{1} \rightarrow \mathbb{R} \times M$ be a $J-$ holomorphic map such that $a(-L-1, t)=R<S=a(L+1, t)$ for all $t \in S^{1}$. Then

$$
\inf _{t} a(-L, t) \leq R+E(f) / \varepsilon, \quad \sup _{t} a(L, t) \geq S-\frac{E(f)}{\varepsilon} .
$$

Proof. If $\inf _{t} a(-L, t) \leq R$, the first inequality holds, so suppose $\inf _{t} a(-L$, $t)>R$. Then apply Corollary 4.21 to the restriction of $f$ to $[-L-1,-L] \times S^{1}$ to obtain

$$
\inf _{t} a(-L, t)-R \leq \frac{E(f)}{\varepsilon} .
$$

The second inequality follows similarly.

\section{Proof of the compactness theorem}

Now we return to the setup of Section 2, which we briefly recall. We are given a closed connected symplectic manifold $(X, \omega)$ and a closed stable hypersurface $\left(M, \omega_{M}:=\left.\omega\right|_{M}, \lambda\right)$ in $X$. Fix $\varepsilon>0$ such that $\omega=\omega_{M}+d(r \lambda)$ on $[-\varepsilon, \varepsilon] \times M$. Fix a tamed almost complex structure $J$ on $(X, \omega)$ whose restriction $J_{M}$ to $[-\varepsilon, \varepsilon] \times M$ is $\omega_{M}$-tamed with $\lambda_{J}=\lambda$. Define the sequence of manifolds $\left(X_{k}, J_{k}, \omega_{\phi_{k}}\right)$ as in Section 2. Recall that $\omega_{\phi_{k}}$ depends on the choice of a diffeomorphism $\phi_{k}:[-k-\varepsilon, \varepsilon] \rightarrow[-\varepsilon, \varepsilon]$ with $\phi^{\prime} \equiv 1$ near the end points of the interval, but its cohomology class $\left[\omega_{\phi_{k}}\right]$ is independent of this choice. 
5.1. Monotonicity. The metric $g_{M}:=\omega_{M}\left(\cdot, J_{M}.\right)+d r^{2}+\lambda^{2}$ on $\mathbb{R} \times M$ is invariant under translation and under $J_{M}$. Pick a $J$-invariant metric $g$ on $X$ which equals $g_{M}$ on the neck $[-\varepsilon, \varepsilon] \times M$. Equip each $X_{k}$ with the $J_{k^{-}}$ invariant Riemannian metric $g_{k}$ which equals $g_{M}$ on the neck $[-k-\varepsilon, \varepsilon] \times M$ and $g$ on $X_{0}$. Pick $\varepsilon_{1}>0$ smaller than the injectivity radius of $(X, g), \varepsilon$ and $\frac{1}{2}$. Then any ball in $\left(X_{k}, g_{k}\right)$ of radius $\rho \leq \varepsilon_{1}$ is isometric to some ball in $(X, g)$ and the almost complex structures agree under this isometry (which is simply given by a shift in the $\mathbb{R}$-component). Let $\varepsilon_{\mathrm{ML}}, C_{\mathrm{ML}}$ be the constants in the Monotonicity Lemma 3.17 for $(X, J, g)$. Then by the preceding discussion, the Monotonicity Lemma holds on $\left(X_{k}, J_{k}, g_{k}\right)$ with constants

$$
\varepsilon_{0}:=\min \left(\varepsilon_{1}, \varepsilon_{\mathrm{ML}}\right)
$$

and $C_{\mathrm{ML}}$ independent of $k$ :

Lemma 5.1. For any $J_{k}$-holomorphic map $f: \Sigma \rightarrow X_{k}$ from a compact Riemann surface, passing through a point $x$ with $f(\partial \Sigma)$ outside the $g_{k}$-ball $B_{\rho}(x)$ of radius $\rho<\varepsilon_{0}$, we have area $a_{k}(f) \geq C_{\mathrm{ML}} \rho^{2}$.

Since $J_{k}$ is tamed by $\omega$ on $X_{0}$ and by $\omega_{M}$ on the neck, the compactness of $X_{0}$ and $M$ and translation invariance imply

Lemma 5.2. There exist a constant $C_{T}>0$, not depending on $k$, such that

$$
\begin{aligned}
\left(\omega_{M}+s d \lambda+d r \wedge \lambda\right)(v, J v) \geq C_{T}|v|_{g_{k}}^{2} & \text { for } s \in[-\varepsilon, \varepsilon], \\
v \in T([-k-\varepsilon, \varepsilon] \times M) & \\
\omega(v, J v) \geq C_{T}|v|_{g_{k}}^{2} & \text { for } v \in T X_{0} .
\end{aligned}
$$

5.2. Energy estimates. Now let $\Sigma$ be a closed connected Riemann surface and $f:(\Sigma, j) \rightarrow X_{k}$ be a $J_{k}$-holomorphic curve. Define its $\omega$-energy (or area)

$$
E(f):=\int_{\Sigma} f^{*} \omega_{\phi_{k}},
$$

and recall that it does not depend on the choice of the function $\phi_{k}$. Over the set $f^{-1}([-k, 0] \times M)$, we write

$$
f=(a, u): f^{-1}([-k, 0] \times M) \rightarrow \mathbb{R} \times M .
$$

For a subset $A \subset[-k, 0]$, we introduce the abbreviation

$$
a^{-1}(A):=f^{-1}(A \times M) .
$$

Lemma 5.3. The energy of the restriction $\left.f\right|_{a^{-1}([-k, 0])}$ satisfies

$$
\frac{2 \epsilon}{1+2 \epsilon} E\left(\left.f\right|_{a^{-1}([-k, 0])}\right) \leq E^{\varepsilon}\left(\left.f\right|_{a^{-1}([-k, 0])}\right) \leq E(f) .
$$


Moreover, the action of every regular level $R$ of the function $a: a^{-1}$ $([-k, 0]) \rightarrow \mathbb{R}$ satisfies

$$
\int_{a^{-1}(R)} u^{*} \lambda \leq \frac{1}{2 \varepsilon} E(f) .
$$

Proof. Set $C:=f^{-1}([-k, 0] \times M)$. Consider any smooth non-decreasing function $\phi:[-k, 0] \rightarrow(-\varepsilon, \varepsilon)$. Extend $\phi$ to a smooth non-decreasing function $\tilde{\phi}:[-k-\varepsilon, \varepsilon] \rightarrow[-\varepsilon, \varepsilon]$ such that $\tilde{\phi}^{\prime} \equiv 1$ near the end points. Nonnegativity of the integrand $f^{*} \omega_{\tilde{\phi}}$ implies

$$
\int_{C} f^{*} \omega_{\phi} \leq \int_{\Sigma} f^{*} \omega_{\tilde{\phi}}=E(f)
$$

Taking the supremum over all such $\phi$ yields the inequality $E^{\varepsilon}\left(\left.f\right|_{C}\right) \leq E(f)$. The other inequalities now follow from Lemma 4.1 and $\varepsilon<1 / 2$.

Remark 5.4. Lemma 5.3 remains valid if $X$ is a symplectic cobordism and $E(f)$ is the Hofer energy of $f$.

5.3. Bounds on the topology. Consider a smooth map $f: \Sigma \rightarrow X_{k}$ from a surface $\Sigma$. For a regular level $-k+1 \leq R \leq-1$, let $\mathcal{C}_{R}$ be the collection of connected components of $a^{-1}([R, R+1])$ and $a^{-1}([R-1, R])$. We define subsets $\mathcal{C}_{R}^{ \pm} \subset \mathcal{C}_{R}$ as follows: First, we include in $\mathcal{C}_{R}^{+}$all components that meet $a^{-1}(R+1)$, as well as those in $a^{-1}([R, R+1])$ that do not meet $R$. Similarly, we include in $\mathcal{C}_{R}^{-}$all components that meet $a^{-1}(R-1)$, as well as those in $a^{-1}([R-1, R])$ that do not meet $R$. Next, we inductively include in $\mathcal{C}_{R}^{+}$all components that can be connected in $\mathcal{C}_{R}$ to $\mathcal{C}_{R}^{+}$without passing through $\mathcal{C}_{R}^{-}$. Finally, we include all remaining components in $\mathcal{C}_{R}^{-}$.

For regular levels $-k+1 \leq R<S \leq-1$ with $S-R \geq 2$, we define the following subsets of $\Sigma$, see Figure 5 :

$$
\begin{aligned}
& \Sigma_{R}^{S}(f):=a^{-1}([R+1, S-1]) \cup \mathcal{C}_{R}^{+} \cup \mathcal{C}_{S}^{-}, \\
& \Sigma_{S}^{R}(f):=\Sigma \backslash \Sigma_{R}^{S}(f) .
\end{aligned}
$$

Note that in $\Sigma_{R}^{S}$, closed components of $a^{-1}([R-1, R+1])$ that meet $a^{-1}(R)$ are excluded and closed components of $a^{-1}([S-1, S+1])$ that meet $a^{-1}(S)$ are included. All boundary components of $\Sigma_{R}^{S}$ lie in $a^{-1}(R)$ or $a^{-1}(S)$. Moreover, for $-k+1 \leq R<S<T \leq-1$ with $S-R, T-S \geq 2$, we have

$$
\Sigma_{R}^{S}(f) \cup \Sigma_{S}^{T}(f)=\Sigma_{R}^{T}(f), \quad \Sigma_{R}^{S} \cap \Sigma_{S}^{T} \subset a^{-1}(S) .
$$

We will always assume without further mentioning that $R$ and $S$, as well as $R \pm 1$ and $S \pm 1$, are regular values of the function $a: f^{-1}([-k, 0] \times M) \rightarrow \mathbb{R}$ associated to $f$.

Lemma 5.5. For any $J_{k}$-holomorphic curve $f: \Sigma \rightarrow X_{k}$ and regular levels $-k+1 \leq R<S \leq-1$ with $S-R \geq 2$, the number of connected components of each of the sets $\Sigma_{S}^{R}(f)$ and $\Sigma_{R}^{S}(f)$ is at most $2 E(f) / C_{\mathrm{ML}} C_{T} \varepsilon_{0}^{2}$. 
Proof.

(i) Fix a smooth non-decreasing map $\phi:[-k, 0] \rightarrow[-\varepsilon, \varepsilon]$ with $\phi(R)=-\varepsilon$ and $\phi(S)=\varepsilon$ and define the 2 -form $\omega_{\phi}:=\omega_{M}+d(\phi \lambda)$ on $[-k, 0] \times M$ as before. By Lemma 5.3, we have

$$
\int_{\Sigma_{R}^{S}} f^{*} \omega_{\phi} \leq E^{\varepsilon}\left(\left.f\right|_{a^{-1}([-k, 0])}\right) \leq E(f) .
$$

Now let $\Sigma_{0}$ be a connected component of $\Sigma_{R}^{S}(f)$. We construct a point $z_{0} \in \Sigma_{0}$ as follows. If $\Sigma_{0}$ has a boundary component on level $R$, we choose $z_{0} \in \Sigma_{0}$ on level $R+1 / 2$ (which exists by definition of $\Sigma_{R}^{S}$ ). If $\Sigma_{0}$ has no boundary component on level $R$ but one on level $S$, we choose $z_{0} \in \Sigma_{0}$ on level $S-1 / 2$. If $\Sigma_{0}$ has no boundary, pick any $z_{0} \in \Sigma_{0}$. Let $B$ be the $g_{k}$-ball around $b:=f\left(z_{0}\right)$ of radius $\varepsilon_{0}$. The choice of $z_{0}$ and $\varepsilon_{0} \leq 1 / 2$ implies $f\left(\partial \Sigma_{0}\right) \cap B=\emptyset$. Hence Lemma 5.1 yields

$$
\operatorname{area}_{g_{k}}\left(\left.f\right|_{\Sigma_{0}}\right) \geq C_{\mathrm{ML}} \varepsilon_{0}^{2} .
$$

Now assume first that $\partial \Sigma_{0} \neq \emptyset$. Pick a smooth non-decreasing map $\phi_{0}:[-k, 0] \rightarrow[-\varepsilon, \varepsilon]$ with $\phi_{0}(R)=-\varepsilon$ and $\phi_{0}(S)=\varepsilon$ such that $\phi_{0}(r)=r-b$ for $r \in\left[b-\varepsilon_{0}, b+\varepsilon_{0}\right]$ (this is possible because $\varepsilon_{0}<\varepsilon$ ). Then $\omega_{\phi_{0}}=\omega_{M}+d((r-b) \lambda)$ on $B$, so Lemma 5.2 yields $\omega_{\phi_{0}}\left(v, J_{k} v\right) \geq$ $C_{T}|v|_{g_{k}}^{2}$ for $v \in T B$, which in view of $f^{*} \omega_{\phi_{0}} \geq 0$ implies

$$
\int_{\Sigma_{0}} f^{*} \omega_{\phi}=\int_{\Sigma_{0}} f^{*} \omega_{\phi_{0}} \geq C_{T} C_{\mathrm{ML}} \varepsilon_{0}^{2}
$$

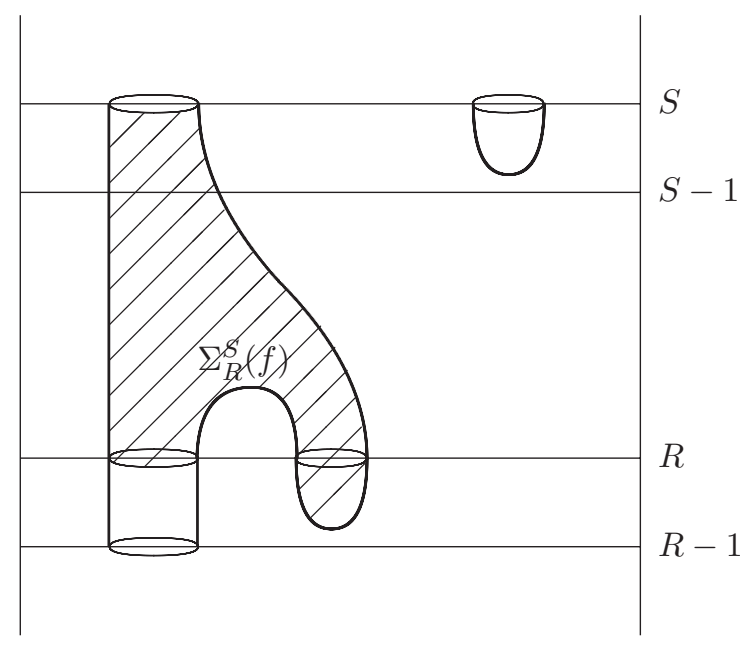

Figure 5. Ignoring small "stalactites" in $\Sigma_{R}^{S}(f)$. 
Here the first equality follows from Stokes' theorem in view of $\phi(R)=$ $\phi_{0}(R)$ and $\phi(S)=\phi_{0}(S)$. If $\Sigma_{0}$ is closed, we take $\phi_{0}:[b-\varepsilon, b+\varepsilon] \rightarrow$ $[-\varepsilon, \varepsilon], \phi_{0}(r):=r-b$, and obtain the same estimate. Thus for any connected component $\Sigma_{0}$ of $\Sigma_{R}^{S}$, we have

$$
\int_{\Sigma_{0}} f^{*} \omega_{\phi} \geq C_{T} C_{\mathrm{ML}} \varepsilon_{0}^{2}
$$

Since $\int_{\Sigma_{R}^{S}} f^{*} \omega_{\phi} \leq E(f)$, the number of connected components cannot exceed $E(f) / C_{T} C_{\mathrm{ML}} \varepsilon_{0}^{2}$.

(ii) The proof for $\Sigma_{S}^{R}(f)$ works similarly. Here we choose a surjective nondecreasing map $\phi:[-k, 0] \rightarrow[-\varepsilon, \varepsilon]$ with $\phi(R)=\phi(S)=0$. If $\partial \Sigma_{0} \neq \emptyset$, we choose $z_{0} \in \Sigma_{0}$ such that $a\left(z_{0}\right)=R-1 / 2$ or $a\left(z_{0}\right)=S+1 / 2$. Thus this time, we obtain a bound given by $2 E(f) / C_{T} C_{\mathrm{ML}} \varepsilon_{0}^{2}$.

Let $-k+1 \leq R_{0} \leq-1$. A subset $\Sigma_{0} \subset \Sigma$ is called an essential local minimum on level $R_{0}$ of $f: \Sigma \rightarrow X_{k}$ if $\Sigma_{0}$ is a connected component of $a^{-1}\left(\left[-k, R_{0}+1\right]\right)$ and $R_{0}=\min _{\Sigma_{0}} a$. See Figure 6 .

Similarly, an essential local maximum on level $R_{0}$ is a connected component $\Sigma_{0}$ of $a^{-1}\left(\left[R_{0}-1,0\right]\right)$ such that $R_{0}=\max _{\Sigma_{0}} a$.

Note that a closed component $\Sigma_{0}$ of $a^{-1}([-k+1,-1])$ gives rise to both an essential local minimum and maximum.

Lemma 5.6. For any $J_{k}$-holomorphic curve $f: \Sigma \rightarrow X_{k}$, the number of essential local minima and maxima is bounded above by $E(f) / C_{T} C_{\mathrm{ML}} \varepsilon_{0}^{2}$.

Proof. The proof is very similar to that of Lemma 5.5. Consider a point on an essential local minimum or maximum $\Sigma_{0}$ with $a\left(z_{0}\right)=R_{0}$ and let $B$ be the $g_{k}$-ball of radius $\varepsilon_{0}$ around $f(z)$. Pick a smooth non-decreasing map $\phi:[-k, 0] \rightarrow[-\varepsilon, \varepsilon]$ with $\phi(-k)=-\varepsilon$ and $\phi(0)=\varepsilon$ such that $\phi(r)=r-R_{0}$ for $r \in\left[R_{0}-\varepsilon_{0}, R_{0}+\varepsilon_{0}\right]$. As in the proof of Lemma 5.5, we find

$$
C_{T} C_{\mathrm{ML}} \varepsilon_{0}^{2} \leq \int_{\Sigma_{0}} f^{*} \omega_{\phi} \leq E(f)
$$

Choosing disjoint balls around all local minima and maxima, this bounds the number of essential local minima and maxima by $E(f) / C_{T} C_{\mathrm{ML}} \varepsilon_{0}^{2}$.

Finally, we need to bound the Euler characteristic $\chi$ of the sets $\Sigma_{S}^{R}(f)$ and $\Sigma_{R}^{S}(f)$. Recall that the Euler characteristic of a compact connected oriented surface $\Sigma_{0}$ of genus $g$ with $n$ boundary components is given by

$$
\chi\left(\Sigma_{0}\right)=2-2 g-n .
$$

In particular, $\chi\left(\Sigma_{0}\right) \leq 2$ with equality iff $\Sigma_{0}$ is the sphere; $\chi\left(\Sigma_{0}\right)=1$ iff $\Sigma_{0}$ is the disk; $\chi\left(\Sigma_{0}\right)=0$ iff $\Sigma_{0}$ is the torus or the cylinder. The surface $\Sigma_{0} \cup \Sigma_{1}$ 
obtained by gluing two surfaces $\Sigma_{0}, \Sigma_{1}$ along some of their boundary circles has Euler characteristic

$$
\chi\left(\Sigma_{0} \cup \Sigma_{1}\right)=\chi\left(\Sigma_{0}\right)+\chi\left(\Sigma_{1}\right) .
$$

From now on, we assume that $\Sigma$ is connected and $E(f) \leq E_{0}$. Set

$$
N_{0}:=2 \max \left(\frac{E_{0}}{C_{T} C_{\mathrm{ML}} \varepsilon_{0}^{2}, 1}\right) .
$$

Lemma 5.7. With the notation of Lemma 5.5, we have the following estimates for the Euler characteristic:

$$
\begin{aligned}
& \chi(\Sigma)-N_{0} \leq \chi\left(\Sigma_{S}^{R}(f)\right) \leq N_{0}, \\
& \chi(\Sigma)-N_{0} \leq \chi\left(\Sigma_{R}^{S}(f)\right) \leq N_{0} .
\end{aligned}
$$

Proof. By Lemma 5.5, $\Sigma_{S}^{R}$ has at most $N_{0}$ components. If one component is a sphere, then it equals $\Sigma$ because $\Sigma$ is connected, so $\chi\left(\Sigma_{S}^{R}\right) \leq 2 \leq N_{0}$ in this case. Otherwise all components have Euler characteristic at most 1, and again $\chi\left(\Sigma_{S}^{R}\right) \leq N_{0}$. The other upper estimate follows similarly. The lower estimates follow from $\chi(\Sigma)=\chi\left(\Sigma_{S}^{R}\right)+\chi\left(\Sigma_{R}^{S}\right) \leq \chi\left(\Sigma_{S}^{R}\right)+N_{0}$ and similarly for the other case.

Lemma 5.5 and Lemma 5.7 show that the number of possible topological types of each of the sets $\Sigma_{S}^{R}(f)$ and $\Sigma_{R}^{S}(f)$ is uniformly bounded. To see this, note that the genus of each of the sets is bounded above by the genus of $\Sigma$, thus the bounds on the Euler characteristic yield bounds on the number of boundary components. The following lemma gives a more precise control of the topology.

Lemma 5.8. With the notation of Lemma 5.5, we have

$$
\chi\left(\Sigma_{R}^{S}(f)\right) \leq \#\left\{\text { essential minima or maxima } \Sigma_{0} \subset \Sigma_{R}^{S}\right\} .
$$

If $\Sigma_{R}^{S}(f)$ contains neither essential minima nor maxima and $\chi\left(\Sigma_{R}^{S}(f)\right)=0$, then $\Sigma_{R}^{S}(f)$ is a disjoint union of cylinders connecting the levels $R$ and $S$.

Proof. Let $\Sigma_{1}$ be a component of $\Sigma_{R}^{S}(f)$ with $\chi\left(\Sigma_{1}\right)>0$. If $\Sigma_{1}$ is a sphere, then it has at least one essential maximum and one essential minimum. If $\Sigma_{1}$ is not a sphere, then $\chi\left(\Sigma_{1}\right)=1$ and $\Sigma_{1}$ is a disk with boundary in $a^{-1}(S)$ (resp. $\left.a^{-1}(R)\right)$. Now $\Sigma_{1} \subset \Sigma_{-k}^{S}(f)$ (resp. $\left.\Sigma_{1} \subset \Sigma_{R}^{0}(f)\right)$ implies that $R_{0}:=\min _{\Sigma_{1}} a \leq S-1$ (resp. $R_{0}:=\max _{\Sigma_{1}} a \geq R+1$ ). Let $\Sigma_{0}$ be a component of $\Sigma_{1} \cap a^{-1}\left(\left[R_{0}, R_{0}+1\right]\right)$ (resp. $\Sigma_{1} \cap a^{-1}\left(\left[R_{0}-1, R_{0}\right]\right)$ on which the minimum (resp. maximum) $R_{0}$ is attained. Since $\Sigma_{1}$ has no lower (resp. upper) boundary, $\Sigma_{0}$ is a connected component of $a^{-1}\left(\left[-k, R_{0}+1\right]\right)$ (resp. $a^{-1}\left(\left[R_{0}-1,0\right]\right)$ and is thus an essential minimum (resp. maximum). This proves the estimate. 
For the last statement, suppose that $\Sigma_{R}^{S}$ contains neither essential minima nor maxima. Then, by the argument above, each component $\Sigma_{1}$ of $\Sigma_{R}^{S}$ has Euler characteristic $\leq 0$. Thus $\chi\left(\Sigma_{R}^{S}\right)=0$ implies $\chi\left(\Sigma_{1}\right)=0$. If $\Sigma_{1}$ had no boundary, it would be an essential local minimum (and maximum), contradicting the hypotheses. So $\Sigma_{1}$ has non-empty boundary and is thus a cylinder. If this cylinder had both boundary components on level $R$ (resp. level $S$ ), it would contain an essential local maximum (resp. minimum), so each cylinder connects the levels $R$ and $S$.

Define a function $\chi_{f}:[-k+1,-3]_{\mathrm{reg}} \rightarrow \mathbb{Z}$ on regular values of $a$ by

$$
\chi_{f}(r):=\chi\left(\Sigma_{r}^{-1}(f)\right) .
$$

This function will play an important role in the compactness proof. It is bounded by Lemma 5.7. We call a value $r \in[-k+1,-3]$ an upward jump if

$$
h_{+}(r):=\limsup _{S \searrow r} \chi_{f}(S)-\liminf _{R \nearrow_{r}} \chi_{f}(R)>0,
$$

and a downward jump if

$$
h_{-}(r):=\limsup _{R \nearrow r} \chi_{f}(R)-\liminf _{S \searrow r} \chi_{f}(S)>0 .
$$

Here the infima and suprema are taken over regular values of the function $a$. The numbers $h_{+}(r)$ and $h_{-}(r)$ are called the height of the jump, see Figure 6.

Lemma 5.9. The height of a downward jump of the function $\chi_{f}$ at $r \in$ $[-k+1,-3]$ is at most the number of essential local minima on levels $r$ and $r-1$ plus the number of essential local maxima on level $r+1$. The total height of all downward jumps, in particular their number, is at most $N_{0}$. The total height of all upward jumps, in particular their number, is at most $3 N_{0}-\chi(\Sigma)$.

Proof. Consider regular values $-k+1<R<S<-3$. By construction, we have $\Sigma_{S}^{-1}(f) \subset \Sigma_{R}^{-1}(f)$. Hence the difference $\chi_{f}(S)-\chi_{f}(R)$ equals the sum of $-\chi(C)$ over all connected components $C$ of $\Sigma_{R}^{-1}(f) \backslash \Sigma_{S}^{-1}(f)$. The contribution $-\chi(C)$ of a connected component $C$ is negative only in the case that $C$ is either a sphere or a disk. If $C$ is a sphere, we must have $R<\min _{C} a<S$ (the first condition ensuring $C \subset \Sigma_{R}^{-1}(f)$ and the second one $\left.C \not \subset \Sigma_{S}^{-1}(f)\right)$, so $C$ contains an essential local miminum in the interval $[R, S]$. If $C$ is a disk, its boundary must lie on $\{R\} \times M$ or $\{S\} \times M$. In the first case, we must have $R+1<\max _{C} a<S+1$ (the first condition ensuring $C \subset \Sigma_{R}^{-1}(f)$ and the second one $\left.C \not \subset \Sigma_{S}^{-1}(f)\right)$, so $C$ contains an essential local maximum in the interval $[R+1, S+1]$. Similarly, in the second case we must have $R-1<\min _{C} a<S-1$, so $C$ contains an essential local minimum in the interval $[R-1, S-1]$. Choosing suitable sequences of regular values $R \nearrow r$ and $S \searrow r$, this proves the first assertion of the lemma. 


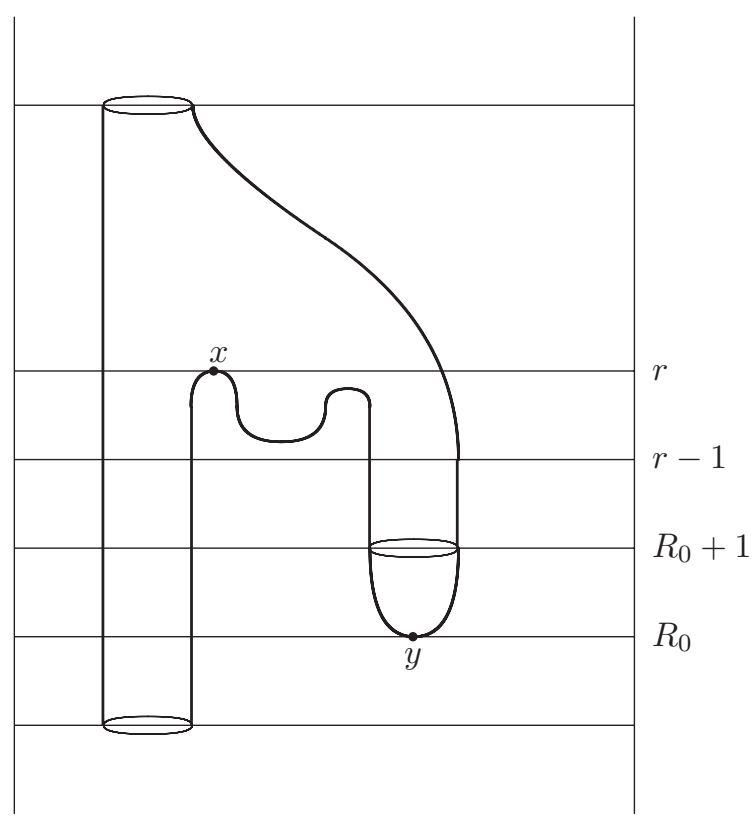

Figure 6. An upward jump at $r$ due to a non-extremal critical point $x$ and a downward jump at $R_{0}+1$ due to an essential local minimum $y$. Notice that no jump occurs at the other local minimum.

It follows that the total height of all downward jumps is bounded by twice the number of essential local extrema (maxima and minima), which is at most $N_{0}$ by Lemma 5.6. Since by Lemma 5.7, the values of $\chi_{f}(r)$ lie in an interval of length $2 N_{0}-\chi(\Sigma)$, the total height of all upward jumps is at most $3 N_{0}-\chi(\Sigma)$.

In view of the preceding lemma, we can extend $\chi_{f}$ from the regular values to a function $\chi_{f}:[-k+1,-3] \rightarrow \mathbb{Z}$ which is locally constant except at the finitely many jumps.

5.4. Detecting the levels. Let now $\left(f_{k}\right)_{k \in \mathbb{N}}$ be a sequence of $J_{k}$-holomorphic maps $f_{k}:\left(\Sigma_{k}, j_{k}\right) \rightarrow X_{k}$ of the same genus and with uniformly bounded energy $E\left(f_{k}\right) \leq E_{0}$. We will screen the neck of $X_{k}$ for the essential parts $f_{k}$ which have the potential to give rise to non-trivial components of the limit. This part of the proof is borrowed from the Floer theory. The new feature here is that, in addition to non-trivial action, we also have to detect non-trivial topology. 
For each $k$, consider the functions

$$
\begin{aligned}
& \chi_{k}:[-k+1,-3] \rightarrow \mathbb{Z}, \quad r \longmapsto \chi\left(\Sigma_{r}^{-1}\left(f_{k}\right)\right), \\
& \mathcal{A}_{k}:[-k, 0] \rightarrow \mathbb{R}, \quad r \longmapsto \int_{f_{k}^{-1}([r, 0] \times M)} f_{k}^{*} \omega_{M} .
\end{aligned}
$$

More precisely, both functions are first defined on regular values and then extended such that $\mathcal{A}_{k}$ becomes continuous and $\chi_{k}$ is locally constant except for finitely many jumps as above. Note that the function $\mathcal{A}_{k}$ is strictly decreasing because $f_{k}^{*} \omega_{M} \geq 0$. Recall the definition of $\beta_{0}$ from Section 4 .

Call a level $r \in[-k, 0]$ essential for $f_{k}$ if it satisfies one of the following conditions:

- $r=0$ or $r=-k$;

- $\mathcal{A}_{k}(r)=j \beta_{0}$ for some $j \in \mathbb{Z}$;

- $f_{k}$ has an essential minimum on the level $r-1$ or an essential maximum on the level $r+1$;

- $\chi_{k}$ has a jump at $r$;

- $f_{k}$ has a marked point on level $r$.

In view of Lemma 5.6, Lemma 5.9 and the energy bound $0 \leq \mathcal{A}_{k}(r) \leq E_{0}$, the number of essential levels of $f_{k}$ is bounded by a constant independent of $k$. After passing to a subsequence, we may assume that this number is constant, say $N^{\prime}+2$. For each $k$ order, the essential levels as $-k=s_{k}^{(0)}<$ $\cdots<s_{k}^{\left(N^{\prime}+1\right)}=0$. Call two levels $\nu$ and $\mu$ equivalent if $s_{k}^{(\nu)}-s_{k}^{(\mu)}$ is uniformly bounded for all $k$. Retain only one level in each equivalence class, including the highest and lowest level. Pass to a subsequence such that the number of equivalence classes is constant, say $N+2$ for some $N \leq N^{\prime}$. Order the remaining levels again as

$$
-k=r_{k}^{(0)}<\cdots<r_{k}^{(N+1)}=0 .
$$

By construction, $\lim _{k \rightarrow \infty} r_{k}^{(\nu+1)}-r_{k}^{(\nu)}=\infty$. Since every equivalence class above contained only finitely many levels, there exists an $R_{0}>0$ such that all the dropped levels $s_{k}^{(\mu)}$ that were equivalent to $r_{k}^{(\nu)}$ are contained in the interval $\left(r_{k}^{(\nu)}-R_{0}, r_{k}^{(\nu)}+R_{0}\right)$.

For $k>2 R_{0}$, define the essential regions $\mathcal{E}_{k}^{(\nu)}$ and the cylindrical regions $\mathcal{Z}_{k}^{(\nu)}$ by (Figure 7).

$$
\begin{aligned}
& \mathcal{E}_{k}^{(0)}:=\Sigma_{-R_{0}}^{-k+R_{0}}\left(f_{k}\right), \quad \mathcal{E}_{k}^{(\nu)}:=\Sigma_{r_{k}^{(\nu)}-R_{0}}^{r_{k}^{(\nu)}+R_{0}}\left(f_{k}\right), \quad \nu=1, \ldots, N, \\
& \mathcal{Z}_{k}^{(\nu)}:=\Sigma_{r_{k}^{(\nu)}+R_{0}}^{r^{(\nu+1)}-R_{0}}\left(f_{k}\right), \quad \nu=0, \ldots, N .
\end{aligned}
$$




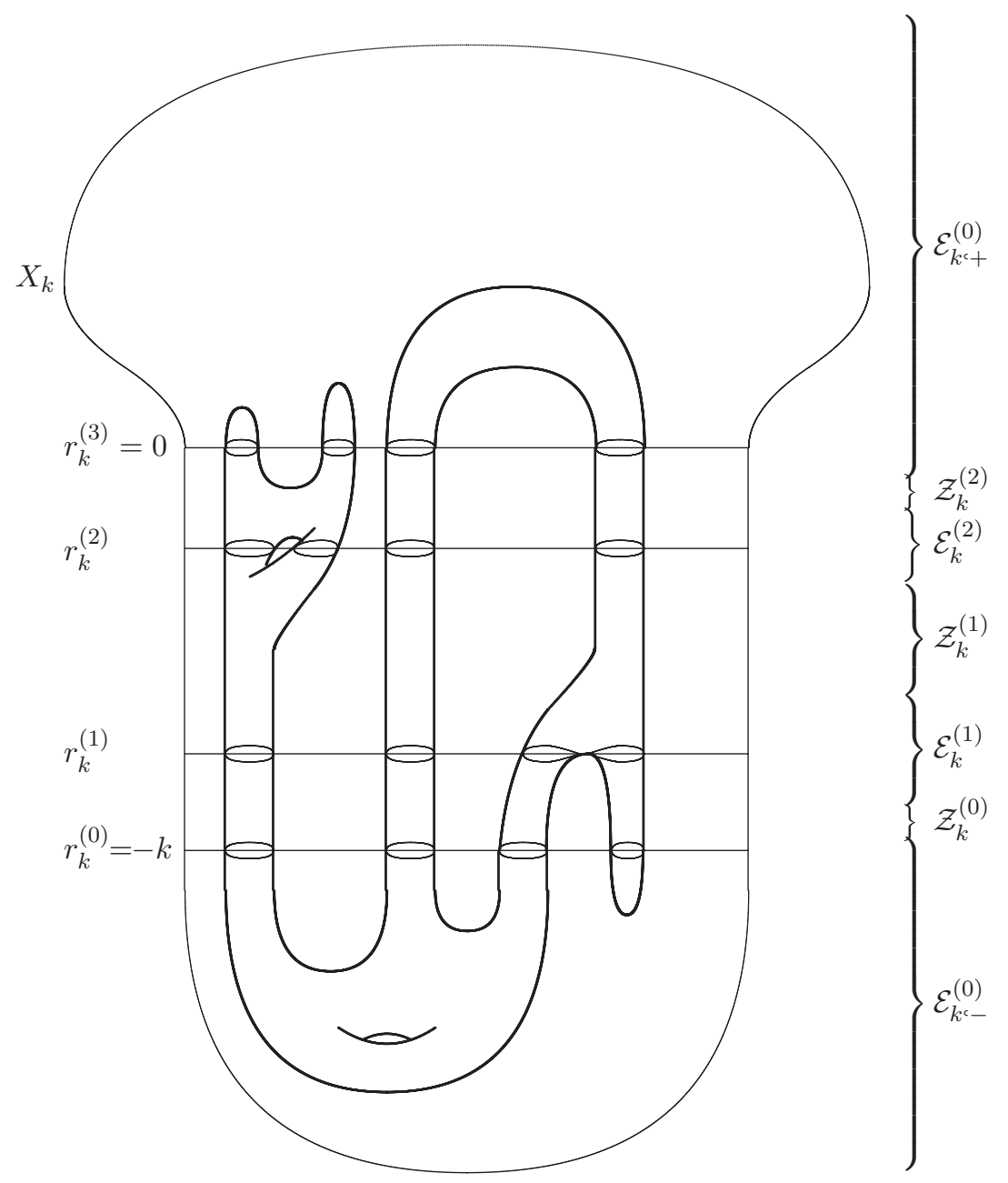

Figure 7. Essential and cylindrical parts in $\Sigma_{k}$ of $f_{k}$.

By construction, for $k$ sufficiently large, the interval $\left[r_{k}^{(\nu)}+R_{0}, r_{k}^{(\nu+1)}-R_{0}\right]$ contains neither essential minima nor maxima nor jumps of the function $\chi_{k}$. Hence $\chi\left(\mathcal{Z}_{k}^{(\nu)}\right)=\chi_{k}\left(r_{k}^{(\nu)}+R_{0}\right)-\chi_{k}\left(r_{k}^{(\nu+1)}-R_{0}\right)=0$, so by Lemma 5.8 , $\mathcal{Z}_{k}^{(\nu)}$ is a union of cylinders connecting the levels $r_{k}^{(\nu)}+R_{0}$ and $r_{k}^{(\nu+1)}-R_{0}$. We summarize this discussion in the following lemma.

Lemma 5.10. There exist constants $N$ and $R_{0}$, independent of $k$, and levels $-k=r_{k}^{(0)}<\cdots<r_{k}^{(N+1)}=0$ with the following properties.

(i) $\lim _{k \rightarrow \infty} r_{k}^{(\nu+1)}-r_{k}^{(\nu)}=\infty$ for $\nu=0, \ldots, N$. 
(ii) For $k$ sufficiently large and $\nu=0, \ldots, N$, the region $\mathcal{Z}_{k}^{(\nu)}$ is a union of at most $N_{0}$ cylinders (with $N_{0}$ as defined before Lemma 5.7) connecting the levels $r_{k}^{(\nu)}+R_{0}$ and $r_{k}^{(\nu+1)}-R_{0}$.

(iii) For every $\nu=0, \ldots, N$, there exists a $j \in \mathbb{Z}$ such that for sufficiently large $k$, the area in $\mathcal{Z}_{k}^{(\nu)}$ satisfies

$$
j \beta_{0} \leq \mathcal{A}_{k}\left(r_{k}^{(\nu)}+R_{0}\right) \leq \mathcal{A}_{k}\left(r_{k}^{(\nu+1)}-R_{0}\right) \leq(j+1) \beta_{0} .
$$

Bookmark for later that we have already obtained the number $N$ of levels of the limit curve.

Corollary 5.11. In Lemma 5.10, after increasing $R_{0}$ and passing to a subsequence, we may further assume the following properties.

(i) The topology of the surfaces $\mathcal{E}_{k}^{(\nu)}$ and $\mathcal{Z}_{k}^{(\nu)}$ is independent of $k$.

(ii) The conformal modulus of each component of $\mathcal{Z}_{k}^{(\nu)}$ tends to infinity as $k \rightarrow \infty$.

Proof.

(i) It follows from Lemma 5.5 and Lemma 5.7, see the discussion following Lemma 5.7.

(ii) It follows from Lemma 4.20 and the upper bound on the action in Lemma 5.3.

5.5. Decomposing the domains. Next we will slightly modify the regions $\mathcal{E}_{k}$ and $\mathcal{Z}_{k}$ to make them fit into the setup of Sections 3 and 4 . Let $\beta_{0}>0$ be the constant defined in Section 4 and $\ell>0$ be the constant in Lemma 4.10. Let $L_{0}>1$ be the constant in the remark after Theorem 3.2, where $\varepsilon_{0}, C_{\mathrm{ML}}$ and $c_{\mathrm{SL}}$ are the constants associated to $\left(X_{k}, J_{k}, g_{k}\right)$ (which are independent of $k$ ). Fix some $L \geq L_{0}$.

Consider one connected component $C_{k}$ of a cylindrical region $\mathcal{Z}_{k}^{(\nu)}$. (In the following discussion, $\nu$ is fixed and often suppressed in the notation.) Let $\beta_{k}$ : $\left[-L_{k}-5 L-\ell, L_{k}+5 L+\ell\right] \times S^{1} \rightarrow\left(C_{k}, j_{k}\right)$ be a conformal parameterization from a standard annulus. Consider the $J_{M}$-holomorphic map $h_{k}:=f_{k} \circ \beta_{k}$ : $\left[-L_{k}-5 L-\ell, L_{k}+5 L+\ell\right] \times S^{1} \rightarrow \mathbb{R} \times M$. Denote by $\left\|\nabla h_{k}\right\|$ the norm of the gradient with respect to the Euclidean metric on the cylinder and the metric $g_{M}$ on $\mathbb{R} \times M$ defined at the beginning of this section.

Lemma 5.12. After passing to a subsequence and increasing $R_{0}$, we may assume that for every component of $\mathcal{Z}_{k}^{(\nu)}$, the corresponding maps $h_{k}=$ $\left(c_{k}, w_{k}\right):\left[-L_{k}-5 L-\ell, L_{k}+5 L+\ell\right] \times \mathbb{R} / \mathbb{Z} \rightarrow \mathbb{R} \times M$ have the following properties, with constants $D>0$ and $R_{1} \geq R_{0}$ not depending on $k$ and $\nu$ :

(i) $L_{k} \rightarrow \infty$ as $k \rightarrow \infty$;

(ii) $E\left(h_{k}\right) \leq E_{0} / \varepsilon$ and $E_{\omega_{M}}\left(h_{k}\right) \leq \beta_{0}$;

(iii) $1 / D \leq\left\|\nabla h_{k}(s, t)\right\| \leq D$ for all $(s, t) \in\left[-L_{k}-5 L, L_{k}+5 L\right] \times S^{1}$; 
(iv) $r_{k}^{(\nu)}+R_{0}-1 \leq \inf _{t} c_{k}\left(-L_{k}-1, t\right) \leq \sup _{t} c_{k}\left(-L_{k}, t\right) \leq r_{k}^{(\nu)}+R_{1}$ and $r_{k}^{(\nu+1)}-R_{1} \leq \inf _{t} c_{k}\left(L_{k}, t\right) \leq \sup _{t} c_{k}\left(L_{k}+1, t\right) \leq r_{k}^{(\nu+1)}-R_{0}+1$.

Proof.

(i) It is contained in Corollary 5.11.

(ii) The first statement follows from Lemma 5.3 and the second one from Lemma 5.10 (iii).

(iii) Lemma 4.10 and the choice of $\ell$ yield the lower estimate $\left\|\nabla h_{k}(s, t)\right\| \geq$ $\frac{\partial c_{k}}{\partial s}(s, t) \geq \delta_{0}$ for $s \in\left[-L_{k}-5 L, L_{k}+5 L\right]$. The upper estimate follows from Lemma 4.3 and property (ii) above.

(iv) Set

$$
r_{k}^{-}:=\inf _{t} c_{k}\left(-L_{k}-5 L-\ell+1, t\right), \quad r_{k}^{+}:=\sup _{t} c_{k}\left(L_{k}+5 L+\ell-1, t\right) .
$$

Then Lemma 4.22 with $R=r_{k}^{(\nu)}+R_{0}, S=r_{k}^{(\nu+1)}-R_{0}$ and $E_{1}:=E_{0} / \varepsilon^{2}$ yields

$$
r_{k}^{-} \leq r_{k}^{(\nu)}+R_{0}+E_{1}, \quad r_{k}^{+} \geq r_{k}^{(\nu+1)}-R_{0}-E_{1} .
$$

In particular, $r_{k}^{+}-r_{k}^{-} \rightarrow \infty$ as $k \rightarrow \infty$. Hence the maps $h_{k}$ satisfy hypotheses (B1) and (B2) of Section 4. By Lemma 4.11, a subsequence of the shifted maps $h_{k}^{-}(s, t):=h_{k}\left(s-L_{k}-5 L-\ell+1, t\right)-\left(r_{k}^{-}, 0\right)$ converges in $C_{\mathrm{loc}}^{\infty}$ on $[0, \infty) \times S^{1}$. In particular, their $\mathbb{R}$-components $c_{k}^{-}$satisfy

$$
c_{k}\left(-L_{k}, t\right)-r_{k}^{-}=c_{k}^{-}(5 L+\ell-1, t) \leq \rho
$$

for all sufficiently large $k$ and a constant $\rho$. With the estimate on $r_{k}^{-}$, this yields

$$
c_{k}\left(-L_{k}, t\right) \leq r_{k}^{(\nu)}+R_{0}+E_{1}+\rho=r_{k}^{(\nu)}+R_{1}
$$

for $R_{1}:=R_{0}+E_{1}+\rho$. An analogous argument shows

$$
c_{k}\left(L_{k}, t\right) \geq r_{k}^{(\nu+1)}-R_{0}-E_{1}-\rho=r_{k}^{(\nu+1)}-R_{1} .
$$

For the converse estimates, note that $\beta_{k}\left(-L_{k}-1, t\right) \in \mathcal{Z}_{k}^{(\nu)}$ for all $t$, so by the definition of $\mathcal{Z}_{k}^{(\nu)}$, we have

$$
c_{k}\left(-L_{k}-1, t\right)=a_{k} \circ \beta_{k}\left(-L_{k}-1, t\right) \geq r_{k}^{(\nu)}+R_{0}-1 .
$$

An analogous argument yields $c_{k}\left(L_{k}+1, t\right) \leq r_{k}^{(\nu+1)}-R_{0}+1$. This concludes the proof.

Now we need some notation. Recall that the number of components of $\mathcal{Z}_{k}^{(\nu)}$ is independent of $k$. So we can parameterize the components by the same index set $I^{(\nu)}$ for all $k$ and write

$$
\mathcal{Z}_{k}^{(\nu)}=\bigcup_{i \in I^{(\nu)}} \mathcal{Z}_{k}^{i}
$$


as a disjoint union of cylinders. Each cylinder is parameterized by a conformal diffeomorphism

$$
\beta_{k}^{i}:\left[-L_{k}^{i}-5 L-\ell, L_{k}^{i}+5 L+\ell\right] \times S^{1} \rightarrow \mathcal{Z}_{k}^{i}
$$

as above. Define the slightly smaller cylinders

$$
Z_{k}^{i}:=\beta_{k}^{i}\left(\left[-L_{k}^{i}-1, L_{k}^{i}+1\right] \times S^{1}\right) \subset \mathcal{Z}_{k}^{i}
$$

and set

$$
Z_{k}^{(\nu)}:=\bigcup_{i \in I^{(\nu)}} Z_{k}^{i}, \quad I:=\bigcup_{\nu=1}^{N-1} I^{(\nu)}, \quad Z_{k}:=\bigcup_{i \in I} Z_{k}^{i}=\bigcup_{\nu=1}^{N-1} Z_{k}^{(\nu)} .
$$

Define the slightly larger essential regions

$$
\begin{aligned}
E_{k}^{(\nu)}:= & \mathcal{E}_{k}^{(\nu)} \cup \bigcup_{i \in I^{(\nu)}} \beta_{k}^{i}\left(\left[-L_{k}^{i}-5 L-\ell,-L_{k}^{i}\right] \times S^{1}\right) \\
& \times \cup \bigcup_{i \in I^{(\nu-1)}} \beta_{k}^{i}\left(\left[L_{k}^{i}, L_{k}^{i}+5 L+\ell\right] \times S^{1}\right)
\end{aligned}
$$

and set

$$
E_{k}:=\bigcup_{\nu=1}^{N} E_{k}^{(\nu)}
$$

Note that $\Sigma_{k}=E_{k} \cup Z_{k}$ and

$$
\begin{aligned}
E_{k}^{(\nu)} \cap Z_{k}^{(\nu)} & =\bigcup_{i \in I^{(\nu)}} \beta_{k}^{i}\left(\left[-L_{k}^{i}-1,-L_{k}^{i}\right] \times S^{1}\right), \\
E_{k}^{(\nu)} \cap Z_{k}^{(\nu-1)} & =\bigcup_{i \in I^{(\nu-1)}} \beta_{k}^{i}\left(\left[L_{k}^{i}, L_{k}^{i}+1\right] \times S^{1}\right) .
\end{aligned}
$$

5.6. Essential components. Consider the holomorphic maps $f_{k}:\left(E_{k}\right.$, $\left.j_{k}\right) \rightarrow\left(X_{k}, J_{k}\right)$ on the essential components. Define the maps

$$
\begin{aligned}
f_{k}^{(0)}: E_{k}^{(0)} & \longrightarrow X_{0}, \\
f_{k}^{(\nu)}:=\left.f_{k}\right|_{E_{k}^{(\nu)}}-r_{k}^{(\nu)}: E_{k}^{(\nu)} & \longrightarrow \mathbb{R} \times M, \quad \nu=1, \ldots, N,
\end{aligned}
$$

where $f_{k}^{(0)}$ equals the composition of $\left.f_{k}\right|_{E_{k}^{(0)}}$ with the map $X_{k} \backslash\{-k / 2\} \times M \rightarrow$ $X_{0}$ that shifts by $k$ on $[-k,-k / 2) \times M$.

Lemma 5.13. For every $\nu=0, \ldots, N$, the maps $f_{k}^{(\nu)}$ satisfy hypotheses $(A 1-A 5)$ in Section 3 with constants independent of $k$.

Proof. By Corollary 5.11 (i), for any $g, n^{ \pm}$, the number of connected components with genus $g$ and $n^{+}$positive and $n^{-}$negative boundary components of the regions $E_{k}^{(\nu)}$ is independent of $k$. By Lemma 5.12 (iv), the image of $f_{k}^{(\nu)}$ is contained in the compact region $\left[-R_{1}, R_{1}\right] \times M$ for $\nu=1, \ldots, N$ and 
in $X_{0} \cup\left[-R_{1}, 0\right] \times M \cup\left[0, R_{1}\right] \times M$ for $\nu=0$. By Lemma 5.3, the $g_{k}$-areas of $f_{k}^{(\nu)}$ are uniformly bounded by a constant independent of $k$. This shows hypotheses (A1-A3) with $\mu=g_{k}$.

By construction, we have at each positive boundary component of $E_{k}^{(\nu)}$ an embedded annulus

$$
\beta_{k}^{i-}:[-5 L, 0] \times S^{1} \longrightarrow E_{k}^{(\nu)}, \quad(s, t) \longmapsto \beta_{k}^{i}\left(s-L_{k}^{i}, t\right), \quad i \in I^{(\nu)},
$$

and at each negative component

$$
\beta_{k}^{i+}:[0,5 L] \longrightarrow S^{1} \rightarrow E_{k}^{(\nu)}, \quad(s, t) \longmapsto \beta_{k}^{i}\left(s+L_{k}^{i}, t\right), \quad i \in I^{(\nu-1)} .
$$

By Lemma 5.12 (iii), the gradients of the maps

$$
f_{k}^{(\nu)} \circ \beta_{k}^{i-}:[-5 L, 0] \times S^{1} \rightarrow \mathbb{R} \times M, \quad f_{k}^{(\nu)} \circ \beta_{k}^{i+}:[0,5 L] \times S^{1} \rightarrow \mathbb{R} \times M
$$

are bounded from below and above by positive constants independent of $k$. This shows hypotheses (A4 and A5).

Now Theorem 3.2 implies.

Corollary 5.14. Subsequences of $f_{k}^{(\nu)}$ converge as $k \rightarrow \infty$ to nodal holomorphic curves

$$
\begin{gathered}
f^{(0)}:\left(E^{(0)}, j^{(0)}\right) \longrightarrow X_{0} \cup\left[-R_{1}, 0\right] \times M \cup\left[0, R_{1}\right] \times M, \\
f^{(\nu)}:\left(E^{(\nu)}, j^{(\nu)}\right) \longrightarrow\left[-R_{1}, R_{1}\right] \times M, \quad \nu=1, \ldots, N .
\end{gathered}
$$

To simplify notation, we have dropped all the bars and do not distinguish between $\bar{E}^{(\nu)}$ and $E^{(\nu)}$ etc. Moreover, by starting with a slightly larger region, we may assume that the convergence is up to the boundary of $E^{(\nu)}$. With this understood, there exist diffeomorphisms $\varphi_{k}^{(\nu)}: E_{k}^{(\nu)} \rightarrow E^{(\nu)}$ such that

- $\left(\varphi_{k}^{(\nu)}\right)_{*} j_{k} \rightarrow j^{(\nu)}$ in $C^{\infty}$ (away from the nodes),

- $f_{k}^{(\nu)} \circ\left(\varphi_{k}^{(\nu)}\right)^{-1} \rightarrow f^{(\nu)}$ in $C^{\infty}$ (away from the nodes) and in $C^{0}$ over the nodes.

Moreover, for each boundary component $i \in I^{(\nu)}$ resp. $i \in I^{(\nu-1)}$, the maps

$$
\varphi_{k}^{(\nu)} \circ \beta_{k}^{i-}:[-1,0] \times S^{1} \longrightarrow E^{(\nu)}, \quad \varphi_{k}^{(\nu)} \circ \beta_{k}^{i+}:[0,1] \times S^{1} \longrightarrow E^{(\nu)}
$$

are independent of $k$.

5.7. Cylindrical components. For $i \in I$, consider the $J_{M}$-holomorphic cylinders

$$
h_{k}^{i}:=f_{k} \circ \beta_{k}^{i}:\left[-L_{k}^{i}-1, L_{k}^{i}+1\right] \times S^{1} \rightarrow \mathbb{R} \times M .
$$

Lemma 5.15. For each $i \in I$, the maps $h_{k}^{i}=\left(c_{k}^{i}, w_{k}^{i}\right)$ satisfy hypotheses (B1 and B2) in Section 4 with constants independent of $k$. 
Proof. Hypothesis (B1) follows from Lemma 5.12 (ii). Hypothesis (B2) follows from Lemma 5.12 (i) and (iv) and $r_{k}^{(\nu+1)}-r_{k}^{(\nu)} \rightarrow \infty$ as $k \rightarrow \infty$.

Consider $i \in I^{(\nu)}$. By Lemma 5.12 (with $R_{0} \geq 1$ ), we have

$$
\begin{aligned}
& r_{k}^{i-}:=\inf _{t} c_{k}^{i}\left(-L_{k}^{i}, t\right) \in\left[r_{k}^{(\nu)}, r_{k}^{(\nu)}+R_{1}\right], \\
& r_{k}^{i+}:=\sup _{t} c_{k}^{i}\left(L_{k}^{i}, t\right) \in\left[r_{k}^{(\nu+1)}-R_{1}, r_{k}^{(\nu+1)}\right] .
\end{aligned}
$$

This allows us to replace $r_{k}^{i-}$ by $r_{k}^{(\nu)}$ and $r_{k}^{i+}$ by $r_{k}^{(\nu+1)}$ in the statements in Section 4 . Thus by Lemma 4.11, a subsequence of the shifted maps

$$
h_{k}^{i-}(s, t):=h_{k}^{i}\left(s-L_{k}^{i}, t\right)-r_{k}^{(\nu)}, \quad h_{k}^{i+}(s, t):=h_{k}^{i}\left(s+L_{k}^{i}, t\right)-r_{k}^{(\nu+1)}
$$

converges in $C_{\mathrm{loc}}^{\infty}$ to holomorphic half cylinders

$$
h^{i-}:[0, \infty) \times S^{1} \longrightarrow \mathbb{R} \times M, \quad h^{i+}:(-\infty, 0] \times S^{1} \longrightarrow \mathbb{R} \times M .
$$

By Lemma 4.12, the half cylinders $h^{i-}$ and $h^{i+}$ are asymptotic to the same closed Reeb orbit $\gamma^{i}$.

Pick a sequence of diffeomorphisms

$$
\theta_{k}^{i}:\left[-L_{k}^{i}-1, L_{k}^{i}+1\right] \rightarrow[-2,2]
$$

such that

$$
\theta_{k}^{i}(s)= \begin{cases}s+L_{k}^{i}-1 & \text { for } s \in\left[-L_{k}^{i}-1,-L_{k}^{i}\right] \\ s-L_{k}^{i}+1 & \text { for } s \in\left[L_{k}^{i}, L_{k}^{i}+1\right]\end{cases}
$$

The reason for choosing these slightly larger intervals will become clear below. Moreover, we assume that their restrictions to $\left[-L_{k}^{i}, L_{k}^{i}\right]$ satisfy hypothesis (B3). Thus the shifted maps

$$
\theta_{k}^{i-}(s):=\theta_{k}^{i}\left(s-L_{k}^{i}\right), \quad \theta_{k}^{i+}(s):=\theta_{k}^{i}\left(s+L_{k}^{i}\right)
$$

converge in $C_{\mathrm{loc}}^{\infty}$ to diffeomorphisms

$$
\theta^{i-}:[-1, \infty) \longrightarrow[-2,0), \quad \theta^{i+}:(-\infty, 1] \longrightarrow(0,2]
$$


By abuse of notation, let us denote the maps $\left[-L_{k}^{i}-1, L_{k}^{i}+1\right] \times S^{1} \rightarrow$ $[-2,2] \times S^{1}$ induced by $\theta_{k}^{i}$ by the same letter. As in Section 4 , define

$$
\begin{aligned}
g_{k}^{i} & :=\left(b_{k}^{i}, v_{k}^{i}\right):=h_{k}^{i} \circ\left(\theta_{k}^{i}\right)^{-1}:[-2,2] \times S^{1} \longrightarrow \mathbb{R} \times M, \\
g_{k}^{i-} & :=\left(b_{k}^{i-}, v_{k}^{i-}\right):=h_{k}^{i-} \circ\left(\theta_{k}^{i-}\right)^{-1}:[-2,2] \times S^{1} \longrightarrow \mathbb{R} \times M, \\
g_{k}^{i+} & :=\left(b_{k}^{i+}, v_{k}^{i+}\right):=h_{k}^{i+} \circ\left(\theta_{k}^{i+}\right)^{-1}:[-2,2] \times S^{1} \longrightarrow \mathbb{R} \times M, \\
g^{i-} & :=\left(b^{i-}, v^{i-}\right):=h^{i-} \circ\left(\theta^{i-}\right)^{-1}:[-2,0) \times S^{1} \longrightarrow \mathbb{R} \times M, \\
g^{i+} & :=\left(b^{i+}, v^{i+}\right):=h^{i+} \circ\left(\theta^{i+}\right)^{-1}:(0,2] \times S^{1} \longrightarrow \mathbb{R} \times M, \\
v^{i}(s, t) & := \begin{cases}v^{i-}(s, t) & : s \in[-2,0), \\
\gamma^{i}(t) & : s=0, \\
v^{i+}(s, t) & : s \in(0,2] .\end{cases}
\end{aligned}
$$

Corollary 5.16. For each $i \in I$ and a subsequence of $g_{k}^{i}$, the following holds:

(i) $g_{k}^{i-} \rightarrow g^{i-}$ on $[-2,0) \times S^{1}$ and $g_{k}^{i+} \rightarrow g^{i+}$ on $(0,2] \times S^{1}$ in $C_{\mathrm{loc}}^{\infty}$.

(ii) $v_{k}^{i} \rightarrow v^{i}$ uniformly on $[-2,2] \times S^{1}$.

(iii) For every $R>0$, there exist $\rho>0$ and $K \in \mathbb{N}$ such that $b_{k}^{i}(s, t) \in$ $\left[r_{k}^{(\nu)}+R, r_{k}^{(\nu+1)}-R\right]$ for all $k \geq K$ and $(s, t) \in[-\rho, \rho] \times S^{1}$.

Proof. For the restrictions to $[-1,1] \times S^{1}$, this follows from Lemma 5.13 and Theorem 4.18. Moreover, by Lemma 5.12 (iv), the maps $h_{k}^{i}$ also satisfy hypotheses (B1 and B2) of Section 4 with $L_{k}$ replaced by $\left(L_{k}+1\right)$. Hence the convergence on $[-2,-1] \times S^{1}$ and $[1,2] \times S^{1}$ follows from Lemma 4.11.

5.8. Domain of the limit curve. For the following discussion, see Figure 8.

Define the disjoint unions of standard annuli

$$
\begin{aligned}
C_{k} & :=\coprod_{i \in I}\left[-L_{k}^{i}-1, L_{k}^{i}+1\right] \times S^{1}, \quad C:=\coprod_{i \in I}[-2,2] \times S^{1}, \\
C_{k}^{-} & :=\coprod_{i \in I}\left[-L_{k}^{i}-1,-L_{k}^{i}\right] \times S^{1}, \quad C^{-}:=\coprod_{i \in I}[-2,-1] \times S^{1}, \\
C_{k}^{+} & :=\coprod_{i \in I}\left[L_{k}^{i}, L_{k}^{i}+1\right] \times S^{1}, \quad C^{+}:=\coprod_{i \in I}[1,2] \times S^{1} .
\end{aligned}
$$

The diffeomorphisms $\theta_{k}^{i}:\left[-L_{k}^{i}-1, L_{k}^{i}+1\right] \rightarrow[-2,2]$ induce diffeomorphisms $\theta_{k}: C_{k} \rightarrow C$ which are of the form $(s, t) \mapsto(s+$ const, $t)$ on each component of $C_{k}^{-} \cup C_{k}^{+}$.

Recall that $Z_{k}=\cup_{i \in I} Z_{k}^{i}$ and $E_{k}=\cup_{\nu=0}^{N} E_{k}^{(\nu)}$. The diffeomorphisms $\beta_{k}^{i}:\left[-L_{k}^{i}-1, L_{k}^{i}+1\right] \times S^{1} \rightarrow Z_{k}^{i}$ induce conformal diffeomorphisms

$$
\beta_{k}:\left(C_{k}, i\right) \longrightarrow\left(Z_{k}, j_{k}\right) \text {. }
$$




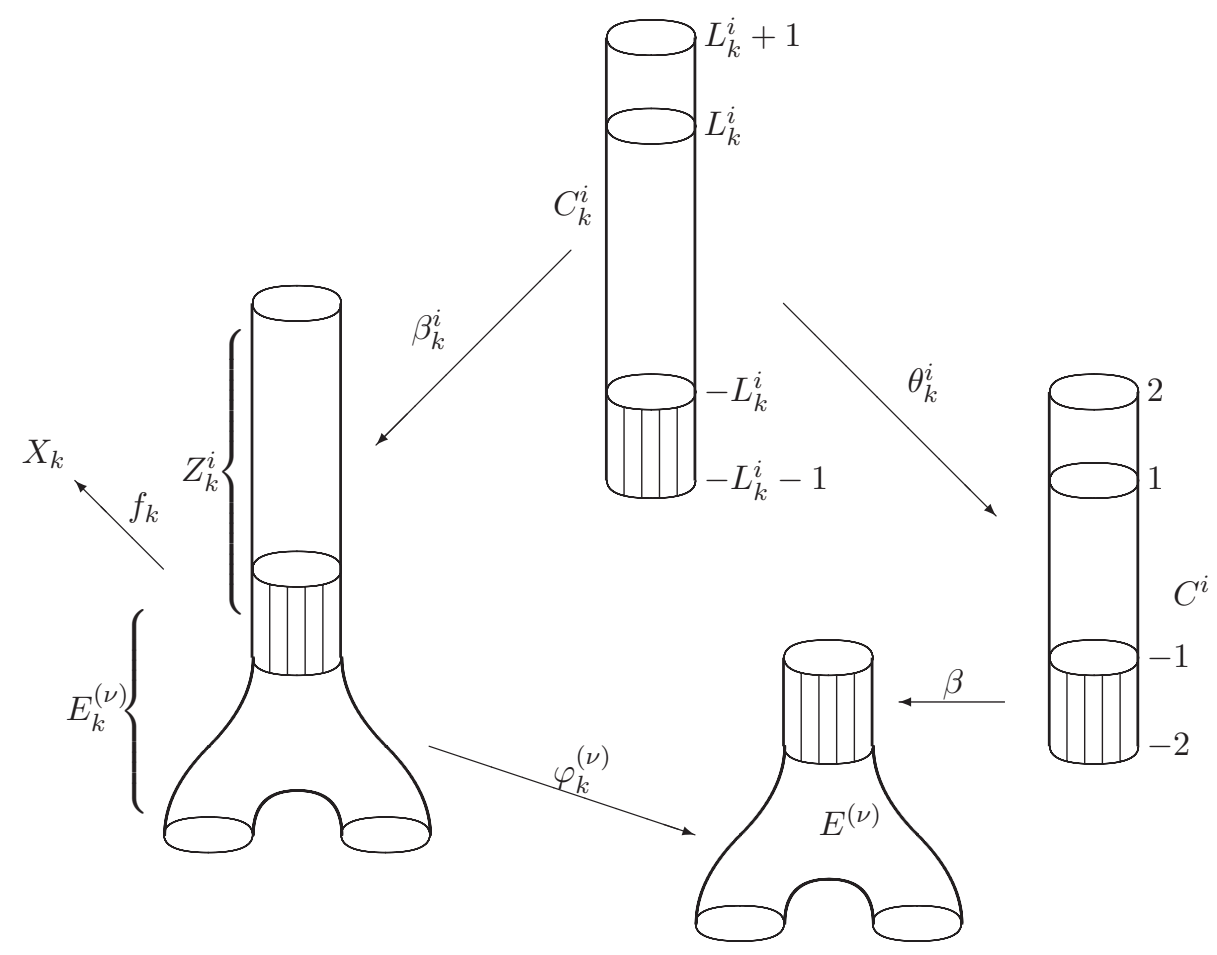

Figure 8. Patching together the cylindrical and essential pieces of $f_{k}$.

Set $Z_{k}^{ \pm}:=\beta_{k}\left(C_{k}^{ \pm}\right)$and note that $E_{k} \cap Z_{k}=Z_{k}^{-} \cup Z_{k}^{+}$. The diffeomorphisms $\varphi_{k}^{(\nu)}: E_{k}^{(\nu)} \rightarrow E^{(\nu)}$ induce diffeomorphisms

$$
\varphi_{k}: E_{k} \longrightarrow E:=\coprod_{\nu=0}^{N} E^{(\nu)}
$$

such that $\left(\varphi_{k}\right)_{*} j_{k}$ converges to the (nodal) conformal structure $j$ on $E$ induced by the $j^{(\nu)}$. Moreover, the embeddings

$$
\beta:=\varphi_{k} \circ \beta_{k} \circ \theta_{k}^{-1}: C^{-} \cup C^{+} \longrightarrow E
$$

are independent of $k$. Glue $E$ and $C$ to obtain a closed surface

$$
\Sigma:=E \cup_{\beta} C .
$$

Since $\beta \circ \theta_{k} \circ \beta_{k}^{-1}=\varphi_{k}$ on $E_{k} \cap Z_{k}$, the maps $\varphi_{k}: E_{k} \rightarrow E$ and $\theta_{k} \circ \beta_{k}^{-1}$ : $Z_{k} \rightarrow C$ fit together to yield diffeomorphisms

$$
\varphi_{k}: \Sigma_{k} \longrightarrow \Sigma
$$


5.9. Convergence of conformal structures. Define the union of disjoint simple loops

$$
\Delta:=\coprod_{i \in I}\{0\} \times S^{1} \subset C .
$$

Viewing $\Delta$ as a subset of $\Sigma$, we define

$$
\Sigma^{*}:=\Sigma \backslash \Delta=: \coprod_{\nu=0}^{N} \Sigma^{(\nu)},
$$

where $\Sigma^{(=n u)}$ is the component containing $E^{(\nu)}$. The diffeomorphisms $\theta^{i-}$ : $[-1, \infty) \rightarrow[-2,0)$ and $\theta^{i+}:(-\infty, 1] \rightarrow(0,2]$ induce a diffeomorphism

$$
\theta:\left(\coprod_{i \in I}[-1, \infty) \times S^{1}\right) \amalg\left(\coprod_{i \in I}(-\infty, 1] \times S^{1}\right) \longrightarrow C \backslash \Delta .
$$

Since $\theta_{k}^{i \pm} \rightarrow \theta^{i \pm}$ in $C_{\mathrm{loc}}^{\infty}$, the conformal structures $\left(\theta_{k}\right)_{*} i$ converge in $C_{\mathrm{loc}}^{\infty}$ on $C \backslash \Delta$ to $\theta_{*} i$. Moreover, by construction, we have $\left(\theta_{k}\right)_{*} i=\theta_{*} i=i$ on $C^{-} \cup C^{+}$. On the other hand, we have $\left(\varphi_{k}\right)_{*} j_{k} \rightarrow j$ in $C_{\mathrm{loc}}^{\infty}$ on $E$ (away from the nodes). Moreover, on $\beta\left(C^{-} \cup C^{+}\right)$, we have

$$
\left(\varphi_{k}\right)_{*} j_{k}=\left(\varphi_{k} \circ \beta_{k}\right)_{*} i=\beta_{*} i
$$

for all $k$, and therefore $j=\beta_{*} i$. This shows that the structures $j$ on $E$ and $\theta_{*} i$ on $C \backslash \Delta$ fit together to a conformal structure $j$ on $\Sigma^{*}$ making it a punctured Riemann surface.

By definition of $\varphi_{k}: \Sigma_{k} \rightarrow \Sigma$, the structure $\left(\varphi_{k}\right)_{*} j_{k}$ on $\Sigma$ is given by $\left(\varphi_{k}\right)_{*} j_{k}$ on $E$ and by $\left(\theta_{k} \circ \beta_{k}^{-1}\right)_{*} j_{k}=\left(\theta_{k}\right)_{*} i$ on $C$. Hence by the preceding discussion, $\left(\varphi_{k}\right)_{*} j_{k} \rightarrow j$ in $C_{\text {loc }}^{\infty}$ on $\Sigma^{*}$. This proves property (a) of Definition 2.7 for the sequence $F_{k}:\left(\Sigma_{k}, j_{k}\right) \rightarrow\left(X_{k}, J_{k}\right)$. Property (b) holds with annuli $\left.\left(\theta_{k}^{i}\right)^{-1}\left(\left[-L_{k}^{i}, L_{k}^{i}\right] \times S^{1}\right]\right):[-1,1] \times S^{1} \rightarrow\left[-L_{k}^{i}, L_{k}^{i}\right] \times S^{1}$.

5.10. Convergence of maps. For $k \in \mathbb{N}$ and $\nu=0, \ldots, N$, define the maps

$$
\begin{aligned}
F_{k} & :=f_{k} \circ \varphi_{k}^{-1}: \Sigma \longrightarrow X_{k}, \\
F_{k}^{(\nu)} & :=f_{k}^{(\nu)} \circ \varphi_{k}^{-1}: \Sigma^{(\nu)} \longrightarrow X_{k}^{(\nu)} .
\end{aligned}
$$

First consider their restriction to an essential component $E^{(\nu)}$. By Corollary 5.14, a subsequence of $F_{k}^{(\nu)}=f_{k}^{(\nu)} \circ\left(\varphi_{k}^{(\nu)}\right)^{-1}: E^{(\nu)} \rightarrow X^{(\nu)}$ converges in the sense of Definition 3.1 to the nodal holomorphic curve $f^{(\nu)}: E^{(\nu)} \rightarrow X^{(\nu)}$.

Next consider a cylindrical component $C^{i}=[-2,2] \times S^{1}$ with $i \in I^{(\nu)}$. Recall that $\varphi_{k}$ is given on $Z_{k}^{i}$ by $\theta_{k}^{i} \circ\left(\beta_{k}^{i}\right)^{-1}: Z_{k}^{i} \rightarrow C^{i}$. This implies

$$
\left.F_{k}\right|_{C^{i}}=f_{k} \circ \beta_{k}^{i} \circ\left(\theta_{k}^{i}\right)^{-1}=h_{k}^{i} \circ\left(\theta_{k}^{i}\right)^{-1}=g_{k}^{i}: C^{i} \longrightarrow \mathbb{R} \times M,
$$

and similarly

$$
\left.F_{k}^{(\nu)}\right|_{C^{i}}=g_{k}^{i-},\left.\quad F_{k}^{(\nu+1)}\right|_{C^{i}}=g_{k}^{i+} .
$$


By Corollary 5.16, for subsequences, we have $C_{\text {loc }}^{\infty}$-convergence $g_{k}^{i-} \rightarrow g^{i-}$ on $[-2,0) \times S^{1}$ and $g_{k}^{i+} \rightarrow g^{i+}$ on $(0,2] \times S^{1}$. By construction, the maps $f^{(\nu)}, g^{i-}$ for $i \in I^{(\nu)}$ and $g^{j+}$ for $j \in I^{(\nu-1)}$ fit together to punctured nodal holomorphic curves $F^{(\nu)}: \Sigma^{(\nu)} \rightarrow X^{(\nu)}$, and a subsequence of $F_{k}^{(\nu)}$ converges to $F^{(\nu)}$ in $C_{\mathrm{loc}}^{\infty}$ on $\Sigma^{(\nu)}$ (away from the nodes). This proves property (c) of Definition 2.7. Denote by $F: \Sigma^{*} \rightarrow X^{*}$ the map induced by the $F^{(\nu)}$ : $\Sigma^{(\nu)} \rightarrow X^{(\nu)}$. By construction, each $F^{(\nu)}$ contains an essential level and is thus stable.

For property (d), consider first the $M$-components $\left.\pi_{M} \circ F_{k}\right|_{C^{i}}=\pi_{M} \circ g_{k}^{i}=$ $v_{k}^{i}: C^{i} \rightarrow M$. By Corollary 5.16, a subsequence of $v_{k}^{i}$ converges uniformly to $v^{i}: C^{i} \rightarrow M$. This shows that $F: \Sigma^{*} \rightarrow X^{*}$ extends to a continuous map $\bar{F}: \Sigma \rightarrow \bar{X}$ and $\pi_{M} \circ F_{k} \rightarrow \pi_{M} \circ \bar{F}$ uniformly on $A^{i}=[-1,1] \times S^{1} \subset C^{i}$. The $\mathbb{R}$-components satisfy $\left.\pi_{\mathbb{R}} \circ F_{k}\right|_{C^{i}}=\pi_{\mathbb{R}} \circ g_{k}^{i}=b_{k}^{i}: C^{i} \rightarrow \mathbb{R}$. Thus the second statement of property (d) follows from Corollary 5.16 (iii).

5.11. Stability. Unfortunately, it may happen that the so-obtained broken holomorphic curve is unstable, i.e., one of the pieces $F^{(\nu)}$ is a disjoint union of cylinders without marked points over a collection of closed Reeb orbits $\Gamma^{(\nu)}$. By construction of the essential levels $r_{k}^{(\nu)}$, this can only happen if there is a $j \in \mathbb{Z}$ such that for all sufficiently large $k$, we have $\mathcal{A}_{k}(r)=j \beta$ for some $r \in\left[r_{k}^{(\nu)}-R_{0}, r_{k}^{(\nu)}+R_{0}\right]$. It follows that the two neighboring pieces $F^{(\nu \pm 1)}$ are both stable: If one of them, say $F^{(\nu+1)}$, was unstable, it would have to be the same collection of orbit cylinders as $F^{(\nu)}$ because the asymptotics agree; on the other hand, it would satisfy $\mathcal{A}_{k}(r)=j^{\prime} \beta$ for some $r \in\left[r_{k}^{(\nu+1)}-R_{0}, r_{k}^{(\nu+1)}+R_{0}\right]$ with $j^{\prime} \neq j \in \mathbb{Z}$, which contradicts the vanishing of $\omega_{M}$ on cylinders over Reeb orbits.

Notice that this discussion remains true for any choice of sufficiently small $\beta_{0}$. Now repeat the selection of essential levels with $\beta_{0}$ replaced by $\beta_{0} / 2$. If a limit piece $F^{(\nu)}$ is unstable, we omit, a posteriori, the level $r_{k}^{(\nu)}$ and go again through the process in the compactness proof. Since by the previous discussion no two adjacent levels become unstable, the $\omega_{M}$-areas of the cylindrical parts are at most $\beta_{0}$. So the compactness proof goes through as before, yielding a stable broken holomorphic curve in the limit.

5.12. Convergence of area. Let $\phi_{k}:[-\varepsilon-k, \varepsilon] \rightarrow[-\varepsilon, \varepsilon]$ be a sequence of diffeomorphisms with $\phi_{k}^{\prime} \equiv 1$ near the boundaries. For $\nu=0, \ldots, N$, define $\phi^{(\nu)}$ and $\phi_{k}^{(\nu)}$ as in Section 2 and assume that $\phi_{k}^{(\nu)} \rightarrow \phi^{(\nu)}$ in $C_{\mathrm{loc}}^{\infty}$. We need to show

$$
\int_{\Sigma_{k}} f_{k}^{*} \omega_{\phi_{k}} \longrightarrow \int_{\Sigma^{*}} F^{*} \omega_{\phi}
$$


With the form $\omega_{\phi_{k}^{(\nu)}}$ on $X_{k}^{(\nu)}$ which equals $\omega_{M}+d\left(\phi_{k}^{(\nu)} \lambda\right)$ on the neck and $\omega$ on $X_{0}$, this is equivalent to

$$
\sum_{\nu=0}^{N} \int_{\Sigma^{(\nu)}}\left(F_{k}^{(\nu)}\right)^{*} \omega_{\phi_{k}^{(\nu)}} \longrightarrow \sum_{\nu=0}^{N} \int_{\Sigma^{(\nu)}}\left(F^{(\nu)}\right)^{*} \omega_{\phi} .
$$

In view of the hypothesis $\phi_{k}^{(\nu)} \rightarrow \phi^{(\nu)}$, we have $\omega_{\phi_{k}^{(\nu)}} \rightarrow \omega_{\phi}$ in $C_{\mathrm{loc}}^{\infty}$ on $X^{(\nu)}$. Together with the $C_{\mathrm{loc}}^{\infty}$-convergence of maps $F_{k}^{(\nu)} \rightarrow F^{(\nu)}$ this implies

$$
\int_{K}\left(F_{k}^{(\nu)}\right)^{*} \omega_{\phi_{k}^{(\nu)}} \longrightarrow \int_{K}\left(F^{(\nu)}\right)^{*} \omega_{\phi}
$$

for every compact subset $K \subset \Sigma^{(\nu)}$. Thus it suffices to show that all the integrals are arbitrarily small outside a sufficiently large compact subset. More precisely, let $[-\delta, \delta] \times S^{1} \subset C^{i}$ be a small annulus around a loop $\Delta^{i} \subset \Delta$ in $\Sigma$ and let $\Sigma^{(\nu)}, \Sigma^{(\nu+1)}$ be the components adjacent to $\Delta^{i}$. Then we need to show that for each $\rho>0$, there exists a $\delta>0$ such that the following statements hold:

(1) $\int_{[-\delta, \delta] \times S^{1}} F^{*} \omega_{\phi}<\rho$;

(2) $\int_{[-\delta, \delta] \times S^{1}}\left(f_{k} \circ \phi_{k}^{-1}\right)^{*} \omega_{\phi_{k}}<\rho$ for all sufficiently large $k$.

Statement (1) follows as in the proof of Lemma 2.6: Let $N^{i} \subset M$ be a neighborhood of the asymptotic Reeb orbit $\gamma^{i}$ at $\Delta^{i}$ on which $\omega_{M}$ is exact, say $\omega_{M}=d \alpha^{i}$. Arguing by Stokes' theorem as in the proof of Lemma 2.6, we find

$$
\int_{[-\delta, \delta] \times S^{1}} F^{*} \omega_{\phi}=\int_{\{\delta\} \times S^{1}} F^{*}\left(\alpha^{i}+\phi \lambda\right)-\int_{\{-\delta\} \times S^{1}} F^{*}\left(\alpha^{i}+\phi \lambda\right) .
$$

Now by the asymptotic behavior, for $\delta$ small, the $M$-component of $\left.F\right|_{\{ \pm \delta\} \times S^{1}}$ is $C^{1}$-close to $\gamma^{i}$ and $\left.\phi \circ F\right|_{\{ \pm \delta\} \times S^{1}}$ is close to a constant $c^{i}$, so the right-hand side becomes $<\rho$.

For statement (2), first note that due to uniform convergence (Corollary 5.16), for small $\delta$, the $M$-projection of $f_{k} \circ \phi_{k}^{-1}\left([-\delta, \delta] \times S^{1}\right)$ is contained in $N^{i}$ for all sufficiently large $k$. Thus Stokes' theorem yields

$$
\begin{aligned}
\int_{[-\delta, \delta] \times S^{1}}\left(f_{k} \circ \phi_{k}^{-1}\right)^{*} \omega_{\phi_{k}}= & \int_{\{\delta\} \times S^{1}}\left(F_{k}^{(\nu+1)}\right)^{*}\left(\alpha^{i}+\phi_{k}^{(\nu+1)} \lambda\right) \\
& -\int_{\{-\delta\} \times S^{1}}\left(F_{k}^{(\nu)}\right)^{*}\left(\alpha^{i}+\phi_{k}^{(\nu)} \lambda\right) .
\end{aligned}
$$

In view of the $C^{\infty}$-convergence $F_{k}^{(\nu)} \rightarrow F^{(\nu)}$ on compact subsets of $\Sigma^{*}$ noted above, the right-hand side of this equation converges to the right-hand side of equation (5.1) as $k \rightarrow \infty$ (with $\delta$ small but fixed) and is therefore $<\rho$ for $k$ sufficiently large. 
This proves property (e) of Theorem 2.9 and hence concludes the proof of the Gromov-Hofer compactness theorem.

5.13. The contact case. Finally, we discuss convergence of area in the case that $M \subset X$ is a hypersurface of contact type which separates $X$ into $\bar{X}_{0}=\bar{X}_{0}^{+} \amalg \bar{X}_{0}^{-}$. Recall from Section 2 the definition of the symplectic forms

$$
\omega_{k}:= \begin{cases}\omega & \text { on } \bar{X}_{0}^{+} \\ d\left(e^{r} \lambda\right) & \text { on }[-k, 0] \times M \\ e^{-k} \omega & \text { on } \bar{X}_{0}^{-} .\end{cases}
$$

The following lemma estimates the $\omega_{k}$-areas from above.

Lemma 5.17. Let $f: \Sigma \rightarrow X_{k}$ be a $J_{k}$-holomorphic curve. Then

$$
\int_{f^{-1}\left(\bar{X}_{0}^{+}\right)} f^{*} \omega_{k} \leq E(f), \quad \int_{f^{-1}\left(\bar{X}_{0}^{-}\right)} f^{*} \omega_{k} \leq e^{-k} E(f) .
$$

Moreover, for any regular levels $-k \leq R<S \leq 0$, we have

$$
\int_{f^{-1}([R, S] \times M)} f^{*} \omega_{k} \leq \frac{e^{S} E(f)}{2 \varepsilon} .
$$

Proof. The first two inequalities follow immediately from non-negativity of $f^{*} \omega$ :

$$
\begin{aligned}
& \int_{f^{-1}\left(\bar{X}_{0}^{+}\right)} f^{*} \omega_{k}=\int_{f^{-1}\left(\bar{X}_{0}^{+}\right)} f^{*} \omega \leq E(f), \\
& \int_{f^{-1}\left(\bar{X}_{0}^{-}\right)} f^{*} \omega_{k}=e^{-k} \int_{f^{-1}\left(\bar{X}_{0}^{+}\right)} f^{*} \omega \leq e^{-k} E(f) .
\end{aligned}
$$

The last inequality follows from positivity of $\int_{f^{-1}(\{R\} \times M)} f^{*} \lambda$ (Lemma 4.1 (a)) and Lemma 5.3:

$$
\begin{aligned}
\int_{f^{-1}([R, S] \times M)} f^{*} \omega_{k} & =\int_{f^{-1}(\{S\} \times M)} e^{S} f^{*} \lambda-\int_{f^{-1}(\{R\} \times M)} e^{R} f^{*} \lambda \\
& \leq e^{S} \int_{f^{-1}(\{S\} \times M)} f^{*} \lambda \leq \frac{e^{S} E(f)}{2 \varepsilon} .
\end{aligned}
$$

Proof of Corollary 2.11. Let $\rho>0$ be given. Lemma 5.17 provides for any $k \in \mathbb{N}$ and $S \in[-k, 0]$ the estimates

$$
\int_{f_{k}^{-1}\left(\bar{X}_{0}^{-}\right)} f_{k}^{*} \omega_{k} \leq e^{-k} E(f), \quad \int_{f_{k}^{-1}([-k, S] \times M)} f_{k}^{*} \omega_{k} \leq \frac{e^{S} E(f)}{2 \varepsilon} .
$$


Since the integrand $f_{k}^{*} \omega_{k}$ is non-negative, this shows the existence of $K \in \mathbb{N}$ and $S<0$ such that

$$
\int_{\Sigma_{k}} f_{k}^{*} \omega_{k}-\rho \leq \int_{f_{k}^{-1}\left([S, 0] \times M \cup \bar{X}_{0}^{+}\right)} f_{k}^{*} \omega_{k} \leq \int_{\Sigma_{k}} f_{k}^{*} \omega_{k}
$$

for all $k \geq K$. Now by construction, we have $f_{k}^{-1}\left([S, 0] \times M \cup \bar{X}_{0}^{+}\right) \subset \Sigma_{+}^{(0)}$ for $k$ sufficiently large. Thus the middle integral equals

$$
\begin{aligned}
\int_{f_{k}^{-1}\left([S, 0] \times M \cup \bar{X}_{0}^{+}\right)} f_{k}^{*} \omega_{k} & =\int_{\left(F_{k}^{(0)}\right)^{-1}\left([S, 0] \times M \cup \bar{X}_{0}^{+}\right)}\left(F_{k}^{(0)}\right)^{*} \omega^{+} \\
& \longrightarrow \int_{k \rightarrow \infty} \int_{\left(F_{+}^{(0)}\right)^{-1}\left([S, 0] \times M \cup \bar{X}_{0}^{+}\right)}\left(F_{+}^{(0)}\right)^{*} \omega^{+}
\end{aligned}
$$

by the $C_{\text {loc }}^{\infty}$-convergence $F_{k}^{(0)} \rightarrow F_{+}^{(0)}$ on $\Sigma_{+}^{(0)}$ and the properness of $F_{+}^{(0)}$. This implies the existence of the limit of areas together with the estimate

$$
\lim _{k \rightarrow \infty} \int_{\Sigma_{k}} f_{k}^{*} \omega_{k}-\rho \leq \int_{\left(F_{+}^{(0)}\right)^{-1}\left([S, 0] \times M \cup \bar{X}_{0}^{+}\right)}\left(F_{+}^{(0)}\right)^{*} \omega^{+} \leq \lim _{k \rightarrow \infty} \int_{\Sigma_{k}} f_{k}^{*} \omega_{k}
$$

for any $\rho>0$. Moreover, the asymptotics of $F_{+}^{(0)}$ imply

$$
\int_{\Sigma_{+}^{(0)}}\left(F_{+}^{(0)}\right)^{*} \omega^{+}-\rho \leq \int_{\left(F_{+}^{(0)}\right)^{-1}\left([S, 0] \times M \cup \bar{X}_{0}^{+}\right)}\left(F_{+}^{(0)}\right)^{*} \omega^{+} \leq \int_{\Sigma_{+}^{(0)}}\left(F_{+}^{(+)}\right)^{*} \omega^{+}
$$

for $S$ sufficiently negative. Together the inequalities imply

$$
\lim _{k \rightarrow \infty} \int_{\Sigma_{k}} f_{k}^{*} \omega_{k}-\rho \leq \int_{\Sigma_{+}^{(0)}}\left(F_{+}^{(0)}\right)^{*} \omega^{+} \leq \lim _{k \rightarrow \infty} \int_{\Sigma_{k}} f_{k}^{*} \omega_{k}+\rho
$$

for arbitrary $\rho>0$. This concludes the proof of Corollary 2.11.

\section{References}

[1] L. Ahlfors, Conformal Invariants: Topics in geometric function theory, McGraw-Hill, New York, 1973.

[2] F. Bourgeois, A Morse-Bott approach to contact homology, PhD thesis, Stanford, 2002.

[3] F. Bourgeois, Y. Eliashberg, H. Hofer, K. Wysocki and E. Zehnder, Compactness results in symplectic field theory, Geom. Topol. 7 (2003), 799-888.

[4] K. Cieliebak and K. Mohnke, Punctured holomorphic curves and Lagrangian embeddings, in preparation.

[5] Y. Eliashberg, A. Givental and H. Hofer, Introduction to symplectic field theory, Geom. Funct. Anal., Special Volume (2000), 560-673.

[6] M. Gromov, Pseudo holomorphic curves in symplectic manifolds, Invent. Math. 82 (1985), 307-347.

[7] H. Hofer, Pseudoholomorphic curves in symplectizations with applications to the Weinstein conjecture in dimension three, Invent. Math. 114(3) (1993), 515-563. 
[8] H. Hofer, K. Wysocki and E. Zehnder, Properties of pseudoholomorphic curves in symplectisations IV: asymptotics with degeneracies, in 'Contact and symplectic geometry' (C. Thomas, ed.) Cambridge University Press, Cambridge, 1996.

[9] H. Hofer, K. Wysocki and E. Zehnder, Finite energy cylinders of small area, Ergodic Theory Dyn. Syst. 22(5) (2002), 1451-1486.

[10] H. Hofer and E. Zehnder, Symplectic invariants and hamiltonian dynamics, Birkhäuser, Basel, 1994.

[11] S. Ivashkovich and V. Shevchishin, Gromov compactness theorem for J-complex curves with boundary, Internat. Math. Res. Notices 22 (2000), 1167-1206

[12] C. Hummel, Gromov's compactness theorem for pseudo-holomorphic curves, Progress in mathematics, 151, Birkhäuser, 1997.

[13] J. Jenkins, On the existence of certain general extremal metrics, Ann. Math. 66 (1957), 440-453.

[14] J. Jost, Compact Riemann surfaces, Springer 2002.

We thank S. Kerckhoff for explaining the theory of extremal length to us, and the referee and editor for valuable comments.

Kai Cieliebak

Mathematisches Institut

LUDWIG-MAXIMILIANS-UNIVERSITÄT

Theresienstrasse 39

80333 MÜnchen, Germany

KLAUS MOHNKE

INSTITUT FÜR MATHEMATIK

Humboldt-Universität Zu Berlin

10099 Berlin, Germany

E-mail address: kai@mathematik.uni-muenchen.de, mohnke@mathematik.hu-berlin.de

Received 02/09/2005, revised 04/19/2006. 